\title{
de Sitter tachyons and related topics
}

\author{
Henri Epstein ${ }^{(a)}$ and Ugo Moschella ${ }^{(a, b)}$ \\ ${ }^{(a)}$ IHES, Bures-sur-Yvette, France \\ ${ }^{(b)}$ DiSat, Università dell'Insubria, Como, and INFN, Sezione di Milano, Italia
}

June 8, 2018

\begin{abstract}
We present a complete study of a family of tachyonic scalar fields living on the de Sitter universe. We show that for an infinite set of discrete values of the negative squared mass the fields exhibit a gauge symmetry and there exists for them a fully acceptable local and covariant quantization similar to the Feynman-Gupta-Bleuler quantization of free QED. For general negative squares masses we also construct positive quantization where the de Sitter symmetry is spontaneously broken. We discuss the sense in which the two quantizations may be considered physically inequivalent even when there is a Lorentz invariant subspace in the second one.
\end{abstract}

\section{Introduction}

The de Sitter universe occupies in contemporary cosmology a place of the greatest importance. The astronomical observations of the last fifteen years have indeed led to the surprising conclusion that the geometry of empty spacetime (namely of spacetime deprived of its matter and radiation content) is more akin to the de Sitter geometry rather than the Minkowski one. Furthermore, if the description of the cosmic fluid provided by the standard $\Lambda \mathrm{CDM}$ cosmological model is correct, matter and radiation must in the future progressively thin out and eventually vanish letting the cosmological constant term alone survive. Therefore, the de Sitter geometry is also the one which the universe approaches asymptotically. This change of paradigm has triggered a renewed interest in the study of de Sitter quantum field theory, both on the foundational side and in view of its applications to cosmology.

In this paper we present a complete study of a family of scalar tachyonic quantum fields whose existence was announced in 1. They are linear Klein-Gordon quantum fields on the de Sitter manifold whose squared masses are negative and take an infinite set of discrete values as follows:

$$
m_{n}^{2}=-n(n+d-1), \quad n=0,1,2, \ldots
$$

here the letter $d$ denotes the dimension of de Sitter space-time.

These models are related to the discrete series of representations of the de Sitter group and they have largely stayed uncovered until now. Already in the early times of de Sitter QFT [2] the possibility of having quantum fields associated to the discrete series had been evoked but this prospect was immediately set aside 2 because of the alleged problems with causality of the corresponding Green functions. Later on however, at least two models belonging to the family (1.1) were drawn to the general attention: the first and more important is the massless scalar field $[3$. This model has been and still is the object of a considerable amount of work and plays a crucial role in the perturbative calculations of cosmological power spectra; the general understanding is that the massless scalar field is substantially different from all other massive fields because of the necessity of breaking the de Sitter symmetry while performing its (canonical) quantization 3, 4, 5, 6]. The second, perhaps less known, model has emerged in the study of the conformal sector of quantum gravity [7, 8, 9] and corresponds to the value $n=1$ (for recent works on the subject see [12, 13, 14, 15] and references 
therein). Also in this case canonical quantization [9] points towards the necessity of breaking the de Sitter symmetry [9, 15, 16.

Here we we will show that both the above examples belong to the infinite family of QFT's labeled by the masses (1.1). All those models share an underlying gauge invariance related to the existence of polynomial regular solutions of the field equations. Because of the existence of such gauge invariance, the properties of local commutativity, de Sitter invariance and positive semidefiniteness [17, 18 cannot hold simultaneously; in other words positive-semi-definiteness and de Sitter invariance of the vacuum expectation values of the fields are incompatible requirements, and, as for gauge QFT's in Minkowski space 19, 20, one is left with the choice of preserving either de Sitter invariance or positivity while quantizing the fields.

In the first case, renouncing the positive-semi-definiteness of the correlation functions not only gives rise to a pseudo-Fock space with an indefinite metric but also to a modification of the KleinGordon equation by the appearance of an anomalous non homogeneous term at the rhs of the field equations:

$$
\left(\square+m_{n}^{2}\right) \phi(x)=\Theta_{n}(x) .
$$

The physical states are identified in the pseudo-Fock space by the vanishing of the matrix elements of that anomalous term:

$$
\left\langle\Psi_{1}, \Theta_{n}(x) \Psi_{2}\right\rangle .
$$

This condition identifies a de Sitter invariant and positive physical subspace where the field equation is weakly re-established: it holds in the mean value sense between physical states. An analogous phenomenon happens in the Feynman-Gupta-Bleuler (covariant) quantization of Quantum Electrodynamics where one is led to modify Gauss' law by adding a term at the rhs:

$$
\partial^{\nu} F_{\mu \nu}=j_{\mu}+\partial_{\mu} \partial^{\nu} A_{\nu}
$$

The physical states are selected by the supplementary condition $\partial^{\nu} A_{\nu}^{-}|p h y s\rangle=0$ and the ordinary Gauss' law holds weakly between physical states.

Nothing of this kind is possible for the tachyonic fields in Minkowski space. Also there it is possible to find a covariant quantization of the tachyon fields [21] that respects causality but there exists no Poincaré invariant positive subspace of the pseudo-Fock space and therefore no direct physical interpretation.

The existence of the above-mentioned gauge invariance was discovered in a Euclidian framework by A. Folacci in [10], where a BRST type quantization was performed. At the moment the relation between that Euclidian BRST approach and the present Gupta-Bleuler type quantization is not completely clear.

In the last part of the paper we explore the alternative possibility and construct all possible positive canonical quantizations of the tachyon fields with spontaneously broken de Sitter symmetry. This is similar to the standard treatment of the massless case as given in [3, 22]; we treat all tachyonic fields

$$
m_{\lambda}^{2}=-\lambda(\lambda+d-1), \quad \lambda \geq 0,
$$

on the same footing. Spontaneous breaking of the de Sitter symmetry means that the commutators are de Sitter invariant distributions and vanish at space like separations while the Wightman functions and the other Green functions are not invariant. The commutator for a given mass parameter $\lambda$ is indeed unique and is the same in all possible quantizations. All quantizations representing the given commutator are therefore canonical by construction; but positive semi-definiteness leads to de Sitter non invariant correlation functions while the invariant quantizations are necessarily non positive. By contrast, in the flat Minkowski space one can find positive quantizations of the tachyons which however do not satisfy local commutativity 24].

Finally we show that in the case $\lambda=n$ it is possible to find under certain circumstances de Sitter invariant subspaces in the reconstructed pseudo-Fock space of the theory which are however not stable under the application of gauge invariant operators. This means that also in the case $\lambda=n$ the positive quantization is physically inequivalent to the covariant quantization [14, 15, 23.

One general lesson to be drawn from these theories is that, even when the cosmological constant is tiny, Minkowskian intuition can be misleading and de Sitter QFT is essentially different from the standard relativistic (Minkowskian) QFT. Other physical consequences of our models, and possible generalizations to higher spin fields 26, 27] will be explored elsewhere. 


\section{Preliminaries}

\section{1 de Sitter space-time: notations and basic definitions.}

The easiest way to look at either the real or the complex de Sitter manifold is to visualize them as subsets of the complex Minkowski manifold with one spacelike dimension more. This allows also a natural description of the fundamental tubular domains encoding the spectral condition of de Sitter quantum field theory [25, 28, 29. Let therefore $M_{d+1}=\mathbf{R}^{d+1}$ be the real $(d+1)$-dimensional Minkowski space-time and $M_{d+1}^{(c)}=\mathbf{C}^{d+1}$ be its complexification, where $d \geq 2$. In a chosen Lorentz frame $\left\{e_{\mu}, \mu=0, \ldots, d\right\}$ the scalar product of two events $x=\left(x^{0}, \vec{x}\right)$ and $y=\left(y^{0}, \vec{y}\right)$ is given by

$$
x \cdot y=x^{0} y^{0}-\vec{x} \cdot \vec{y}=\eta_{\mu \nu} x^{\mu} y^{\nu} .
$$

The future cone $V_{+}$, the future light cone $C_{+}$and the future and past tubes $T_{ \pm}$are defined as follows:

$$
\begin{aligned}
& V_{+}=\left\{x \in M_{d+1}: x \cdot x>0, \quad x^{0}>0\right\} \\
& C_{+}=\left\{x \in M_{d+1}: x \cdot x=0, \quad x^{0} \geq 0\right\}, \\
& T_{ \pm}=\left\{x+i y \in M_{d+1}^{(c)}: y \in \pm V_{+}\right\}
\end{aligned}
$$

The de Sitter space-times with radius $R=1$ and its complexification may be represented as the following one-sheeted hyperboloids:

$$
X_{d}=\left\{x \in M_{d+1} \quad: x \cdot x=-R^{2}=-1\right\} \quad \text { and } \quad X_{d}^{(c)}=\left\{z \in M_{d+1}^{(c)}: z \cdot z=-R^{2}=-1\right\} .
$$

The connected real Lorentz groun $L_{+}^{\uparrow}=S O_{0}(1, d, \mathbf{R})$ leaves $X_{d}$ invariant and acts transitively on it. Similarly the complexification $L_{+}(\mathbf{C})=S O_{0}(1, d, \mathbf{C})$ acts transitively on the complex manifold $X_{d}^{(c)}$. The future and past tuboids $\mathcal{T}_{ \pm}$are the intersections of the ambient tubes $T_{ \pm}$with the complex de Sitter manifold:

$$
\mathcal{T}_{ \pm}=\left\{x+i y \in X_{d}^{(c)}: y \in \pm V_{+}\right\} .
$$

The de Sitter Lorentzian metric $d s_{d}^{2}$ is obtained by restriction of the ambient space-time interval $d s^{2}$ to $X_{d}$. Let us consider in particular the global hyperspherical coordinate system

$$
x(t, \mathbf{x})=\left\{\begin{array}{l}
x^{0}=\operatorname{sh} t \\
\vec{x}=\operatorname{ch} t \mathbf{x}
\end{array}= \begin{cases}x^{0} & =\operatorname{sh} t \\
x^{1} & =\operatorname{ch} t \sin \theta^{1} \\
\vdots & \\
x^{d-1} & =\operatorname{ch} t \cos \theta^{1} \ldots \cos \theta^{d-2} \sin \theta^{d-1} \\
x^{d} & =\operatorname{ch} t \cos \theta^{1} \ldots \cos \theta^{d-2} \cos \theta^{d-1}\end{cases}\right.
$$

where $t \in \mathbf{R}, 0<\theta^{i}<\pi$ and $0<\theta^{d-1}<2 \pi$. It follows that

$$
d s_{d}^{2}=\left.d s^{2}\right|_{X_{d}}=d t^{2}-\operatorname{ch}^{2} t d l^{2} .
$$

where

$$
d l^{2}=\left(d \theta^{1}\right)^{2}+\cos ^{2} \theta^{1}\left(d \theta^{2}\right)^{2}+\cos ^{2} \theta^{1} \cos ^{2} \theta^{2}\left(d \theta^{3}\right)^{2}+\ldots
$$

is the line element on a unit $(d-1)$-dimensional sphere $S^{d-1}$. The hyperspherical Section $S_{0}=\{\xi \in$ $\left.C_{+}: \xi^{0}=1\right\}$ of the asymptotic cone $C_{+}$is also a copy of $S^{d-1}$ which we parametrize as in Eq. (2.7):

$$
\xi(\boldsymbol{\xi})=\left\{\begin{array}{lll}
\xi^{0} & =1 \\
\xi^{1} & = & \sin \theta^{1} \\
\vdots & \\
\xi^{d-1} & =\cos \theta^{1} \ldots \cos \theta^{d-2} \sin \theta^{d-1} \\
\xi^{d} & =\cos \theta^{1} \ldots \cos \theta^{d-2} \cos \theta^{d-1}
\end{array} .\right.
$$

\footnotetext{
${ }^{1}$ The Lorentz group acts as the relativity group of the de Sitter manifold. In this context it may also be called the de Sitter group.
} 
In the above coordinates the various scalar products are written as follows

$$
\begin{aligned}
x(t, \mathbf{x}) \cdot x\left(t^{\prime}, \mathbf{x}^{\prime}\right) & =\operatorname{sh} t \operatorname{sh} t^{\prime}-\operatorname{ch} t \operatorname{ch} t^{\prime} \mathbf{x} \cdot \mathbf{x}^{\prime}, \\
x(t, \mathbf{x}) \cdot \xi(\boldsymbol{\xi}) & =\operatorname{sh} t-\operatorname{ch} t \mathbf{x} \cdot \boldsymbol{\xi}, \\
\xi(\boldsymbol{\xi}) \cdot \xi\left(\boldsymbol{\xi}^{\prime}\right) & =1-\boldsymbol{\xi} \cdot \boldsymbol{\xi}^{\prime}
\end{aligned}
$$

The volume form on the unit sphere is normalized in the standard way:

$$
d \mathbf{x}=\prod_{i=1}^{d-1}\left|\cos \theta^{i}\right|^{d-1-i} d \theta^{i} .
$$

The invariant measure on $X_{d}$ is $d x=2 \delta(x \cdot x+1) d x^{0} \ldots d x^{d}=(\operatorname{ch} t)^{d-1} d t d \mathbf{x}$ where $x$ is of the form (2.7) and $d \mathbf{x}$ is given by (2.14).

\subsection{D'Alembert operators and Klein-Gordon equation}

The Lie algebra $\mathcal{L}$ of $L_{+}^{\uparrow}$ is the vector space of linear operators $A$ such that $(A x) \cdot y+x \cdot(A y)=0$ for all $x$ and $y$ in $M_{d+1}$. Particular elements of $\mathcal{L}$ are $M_{\mu \nu}=-M_{\nu \mu}=e_{\mu} \wedge e_{\nu}$. The regular faithful representation $\mathcal{V}$ of $L_{+}^{\uparrow}$ in the space of $\mathcal{C}^{\infty}$ functions on $M_{d+1}$ or $X_{d}$ is given by

$$
\mathcal{V}(\Lambda) f(x)=f\left(\Lambda^{-1} x\right)
$$

The representatives $\mathcal{M}_{\mu \nu}$ of the generators $M_{\mu \nu}$ are

$$
\mathcal{M}_{\mu \nu} f(x)=\left.\frac{d}{d t} f\left(e^{-t M_{\mu \nu}} x\right)\right|_{t=0}=\left(x_{\mu} \partial_{\nu}-x_{\nu} \partial_{\mu}\right) f(x) \text { on } M_{d+1} .
$$

The de Sitter-d'Alembert operator $\square$ is related to the d'Alembert operator $\square_{\mathrm{Mink}}=\partial^{\mu} \partial_{\mu}$ in the ambient Minkowski space $M_{d+1}$ as follows: if $f$ is a $\mathcal{C}^{\infty}$ function (or a distribution) homogeneous of degree 0 on $M_{d+1}$ then $\square\left(\left.f\right|_{X_{d}}\right)=\left.\left(\square_{\text {Mink }} f\right)\right|_{X_{d}}$ (see e.g. 30]). Let D denote the infinitesimal generator of dilations

$$
(\mathrm{D} f)(x)=x^{\mu} \partial_{\mu} f(x)
$$

The following relation holds on $M_{d+1}$ :

$$
\mathcal{M}^{2}=\mathcal{M}^{\mu \nu} \mathcal{M}_{\mu \nu}=2 x^{2} \square_{\mathrm{Mink}}-2(d-1) \mathrm{D}-2 \mathrm{D}^{2} .
$$

If $f$ and $h$ are $\mathcal{C}^{\infty}$ functions on $M_{d+1}$ and $h$ is Lorentz invariant, then

$$
\mathcal{V}(\Lambda)(h(x) f(x))=h(x) f\left(\Lambda^{-1} x\right), \quad \mathcal{M}_{\mu \nu}(h(x) f(x))=h(x) \mathcal{M}_{\mu \nu} f(x) .
$$

This remains true if $h$ is a Lorentz invariant distribution such as $\delta(x \cdot x+1)$. In particular the operators $\mathcal{V}(\Lambda), \mathcal{M}_{\mu \nu}, \mathcal{M}^{2}$, commute with the restriction to invariant submanifolds of $M_{d+1}$. They also commute with dilations.

Let $f$ be a $\mathcal{C}^{\infty}$ function (or a distribution) defined on the open set of events spacelike w.r.t. the chosen origin

$$
\bar{V}^{c}=\left\{x \in M_{d+1}: x \cdot x<0\right\} ;
$$

if $f$ is homogeneous of degree 0 (i.e. $D f=0$ ) it satisfies $\mathcal{M}^{2} f(x)=2 x^{2} \square_{\text {Mink }} f(x)$, hence $\mathcal{M}^{2}\left(\left.f\right|_{X_{d}}\right)=-2 \square\left(\left.f\right|_{X_{d}}\right)$. Since any $\mathcal{C}^{\infty}$ function (or any distribution) on $X_{d}$ has a unique extension homogeneous of degree 0 in the open set $\bar{V}^{c}$, we have

$$
\square f=-\frac{1}{2} \mathcal{M}^{2} f \quad \text { on } X_{d}
$$

for every $\mathcal{C}^{\infty}$ function or distribution $f$ on $X_{d}$.

Assume now that a $\mathcal{C}^{\infty}$ function $f$ on $\bar{V}^{c}$ is homogeneous of degree $\lambda$ and satisfies the wave equation $\square_{\text {Mink }} f=0$. Eq. (2.18) implies that

$$
\mathcal{M}^{2} f(x)=-2 \lambda(\lambda+d-1) f(x)
$$

and, by 2.21,

$$
(\square-\lambda(\lambda+d-1))\left(\left.f(x)\right|_{X_{d}}\right)=0
$$


i.e. the restriction of $f(x)$ to the de Sitter manifold satisfies the Klein-Gordon equation with complex squared mass

$$
m_{\lambda}^{2}=-\lambda(\lambda+d-1)
$$

Note also that the same is true for a function $f$ homogeneous of degree $(1-d-\lambda)$ i.e. the KleinGordon equation (2.23) is invariant under the involution

$$
\lambda \rightarrow 1-d-\lambda .
$$

This symmetry will play a considerable role in what follows. Conversely, any distribution solving the de Sitter Klein-Gordon equation (2.23) has an extension to $\left\{x \in M_{d+1}: x \cdot x<0\right\}$ which is homogeneous of degree $\lambda$ and satisfies the wave equation. Finally putting together (2.21) and (2.18) shows that for every $\mathcal{C}^{\infty}$ function on on $\bar{V}^{c}$

$$
\square_{d S}\left(f \mid X_{d}\right)=\left(\square_{\mathrm{Mink}}+(d-1) D+D^{2}\right) f \mid X_{d}
$$

\section{3 de Sitter Klein-Gordon fields with complex squared mass}

This following two Sections one constitute a summary of the linear Klein-Gordon quantum field theory on the de Sitter universe. As regards the massive theories of course this is not new 32, 33. 34, 35, 36, 37, 38, 39, 25, 28, 29]; our construction however also describes fields having complex squared masses and is preliminary for the construction of the massless and tachyonic fields of this paper. We will extensively use the hyperspherical harmonics described, e.g. in [40, Chap. XI] and [11. Chap. IX], and the closely connected Gegenbauer polynomials. In the following $\left\{Y_{l M}=Y_{M}^{d, l}\right\}$ are the real hyperspherical harmonics described in Appendix A they are homogeneous polynomials of degree $l$ on $\mathbf{R}^{d}$. For each $l$ their restrictions to the sphere $S^{d-1}$ satisfy

$$
-\triangle_{\mathbf{x}} Y_{l M}(\mathbf{x})=l(l+d-2) Y_{l M}(\mathbf{x}) .
$$

In the spherical coordinate system (2.7) the de Sitter Klein-Gordon equation takes the form

$$
\square \phi-\lambda(\lambda+d-1) \phi=\frac{1}{(\operatorname{ch} t)^{d-1}} \partial_{t}\left((\operatorname{ch} t)^{d-1} \partial_{t} \phi\right)-\frac{1}{\operatorname{ch}^{2} t} \triangle_{\mathbf{x}} \phi-\lambda(\lambda+d-1) \phi=0 .
$$

The parameter $\lambda$ and, consequently, the squared mass $m_{\lambda}^{2}=-\lambda(\lambda+d-1)$ are assumed to be complex numbers; $m_{\lambda}^{2}$ is real and positive in the following special cases:

$$
\begin{aligned}
& \text { either } \quad \lambda=-\frac{d-1}{2}+i \rho, \quad \operatorname{Im} \rho=0, \quad m=\sqrt{\left(\frac{d-1}{2}\right)^{2}+\rho^{2}} \geq \frac{d-1}{2}, \\
& \text { or } \quad \operatorname{Im} \lambda=0, \quad 1-d<\operatorname{Re} \lambda<0, \quad 0<m<\frac{d-1}{2} .
\end{aligned}
$$

If $\phi(t, \mathbf{x})=\phi(x(t, \mathbf{x}))$ is a distribution satisfying equation (3.2) and $\varphi$ is any $\mathcal{C}^{\infty}$ function on the sphere $S^{d-1}$, a general result asserts that the average

$$
\int_{S^{d-1}} \phi(t, \mathbf{x}) \varphi(\mathbf{x}) d \mathbf{x}
$$

is a $\mathcal{C}^{\infty}$ function of the $t$ coordinate; in particular this is true for the function

$$
(\operatorname{ch} t)^{\frac{2-d}{2}} h(t)=\int_{S^{d-1}} \phi(t, \mathbf{x}) Y_{l M}(\mathbf{x}) d \mathbf{x} .
$$

Eq. (3.1) implies that the projectors $V_{l M}$ in $L^{2}\left(S^{d-1}\right)$ with kernel $Y_{l M}(\mathbf{x}) Y_{l M}\left(\mathbf{x}^{\prime}\right)$ and, consequently, their sum $V_{l}=\sum_{M} V_{l M}$ commute with the Laplacian $\Delta_{\mathbf{x}}$. As a consequence the operators

$$
\begin{array}{r}
\left(\mathbf{V}_{l M} \phi\right)(t, \mathbf{x})=\int_{S^{d-1}} Y_{l M}(\mathbf{x}) Y_{l M}\left(\mathbf{x}^{\prime}\right) \phi\left(t, \mathbf{x}^{\prime}\right) d \mathbf{x}^{\prime}, \\
\left(\mathbf{V}_{l} \phi\right)(t, \mathbf{x})=\sum_{M}\left(\mathbf{V}_{l M} \phi\right)(t, \mathbf{x})=\int_{S^{d-1}} V_{l}\left(\mathbf{x}, \mathbf{x}^{\prime}\right) \phi\left(t, x^{\prime}\right) d \mathbf{x}^{\prime},
\end{array}
$$


defined on either $\mathcal{C}^{\infty}\left(X_{d}\right)$ or $\mathcal{D}^{\prime}\left(X_{d}\right)$ are also projectors and commute with $\square$. In the above equation we introduced the kernel

$$
V_{l}\left(\mathbf{x}, \mathbf{x}^{\prime}\right)=\sum_{M} Y_{l M}(\mathbf{x}) Y_{l M}\left(\mathbf{x}^{\prime}\right)=\frac{(2 l+d-2) \Gamma(d / 2)}{2 \pi^{d / 2}(d-2)} C_{l}^{\frac{d-2}{2}}\left(\mathbf{x} \cdot \mathbf{x}^{\prime}\right)
$$

which is nothing but a suitably normalized Gegenbauer polynomial (see Appendix A, (A.6)). Summarizing, if $\phi$ is a solution of the Klein-Gordon equation and $h(t)$ is defined as in Eq. (3.6)

$$
\left(\mathbf{V}_{l M} \phi\right)(x)=(\operatorname{ch} t)^{\frac{2-d}{2}} h(t) Y_{l M}(\mathbf{x})
$$

is also a solution of the Klein-Gordon equation with a definite value of the angular momentum.

Let us now introduce the complex variable $z=-i \operatorname{sh} t$, so that $1-z^{2}=\operatorname{ch}^{2} t$, and denote $h(t)=f(-i \operatorname{sh} t)$. Eq. (3.2) implies that $f$ has to be a solution of the Legendre differential equation:

$$
\left(1-z^{2}\right) f^{\prime \prime}(z)-2 z f^{\prime}(z)+\nu(\nu+1) f(z)-\frac{\mu^{2}}{\left(1-z^{2}\right)} f(z)=0,
$$

with

$$
\nu=\lambda+\kappa, \quad \mu=-(l+\kappa), \quad \kappa=\frac{d-2}{2} .
$$

Any $\mathcal{C}^{\infty}$ solution of Eq. (3.11) which is defined for $z=-i \operatorname{sh} t, t$ real, extends to a holomorphic function with singularities only at $z= \pm 1$ or $\infty$. Two linearly independent solutions are the socalled "Legendre functions on the cut" $\mathbf{P}_{\nu}^{\mu}(z)$ and $\mathbf{Q}_{\nu}^{\mu}(z)$ (see [42, p. 143]). These functions are holomorphic in the cut-plane

$$
\Delta_{2}=\mathbf{C} \backslash(-\infty-1] \cup[1, \infty) .
$$

The function of the first kind $\mathbf{P}_{\nu}^{\mu}(z)$ respects the symmetry (2.25): for all $z \in \Delta_{2}$ it satisfies the identity 42

$$
\mathbf{P}_{\lambda+\kappa}^{\mu}(z)=\mathbf{P}_{\nu}^{\mu}(z)=\mathbf{P}_{-\nu-1}^{\mu}(z)=\mathbf{P}_{1-d-\lambda+\kappa}^{\mu}(z)
$$

The reality conditions

$$
\overline{\mathbf{P}_{\nu}^{\mu}(z)}=\mathbf{P}_{\bar{\nu}}^{\bar{\mu}}(\bar{z}), \quad \overline{\mathbf{Q}_{\nu}^{\mu}(z)}=\mathbf{Q}_{\bar{\nu}}^{\bar{\mu}}(\bar{z})
$$

also hold for all $z \in \Delta_{2}$. If $\mu+\nu$ and $\mu-\nu-1$ are not non-negative integers, $\mathbf{P}_{\nu}^{\mu}(z)$ and $\mathbf{P}_{\nu}^{\mu}(-z)$ also constitute two linearly independent solutions of Eq. (3.11). Therefore, if $(\lambda-l)$ and $-(\lambda+d-1+l)$ are not non-negative integers, the general solution with angular momentum $l$ and "magnetic" multiindex $M$ has the form 2

$$
\phi_{\lambda l M}(t, \mathbf{x})=(\operatorname{ch} t)^{-\kappa}\left[a_{\lambda l M} \mathbf{P}_{\lambda+\kappa}^{-l-\kappa}(i \operatorname{sh} t)+b_{\lambda l M} \mathbf{P}_{\lambda+\kappa}^{-l-\kappa}(-i \operatorname{sh} t)\right] Y_{l M}(\mathbf{x}) .
$$

In the following without loss of generality, we restrict our attention to values of $\lambda$ such that

$$
\operatorname{Re} \lambda+\frac{d-1}{2} \geq 0
$$

\subsection{Normalization - Covariant commutators}

The first task in quantizing a field on any globally hyperbolic curved space-time is to construct the covariant commutator

$$
\left[\phi(x), \phi\left(x^{\prime}\right)\right]=C\left(x, x^{\prime}\right) .
$$

The commutator is a c-number and has therefore an algebraic character; it does not depend on anything but the Lorentzian structure of the space-time manifold (see e.g. [43, 44, 45]). In our case, given a complex $\lambda$ we want to construct a bivariate distribution solving the Klein-Gordon equation in each variable, with initial conditions set by the the equal-time canonical commutation relations; in the spherical coordinate system

$$
\begin{aligned}
& {\left[\square_{x}-\lambda(\lambda+d-1)\right] C_{\lambda}\left(x, x^{\prime}\right)=\left[\square_{x^{\prime}}-\lambda(\lambda+d-1)\right] C_{\lambda}\left(x, x^{\prime}\right)=0,} \\
& \left.C_{\lambda}\left(t, \mathbf{x}, t^{\prime}, \mathbf{x}^{\prime}\right)\right|_{t=t^{\prime}}=0,\left.\quad \frac{\partial}{\partial t^{\prime}} C_{\lambda}\left(t, \mathbf{x}, t^{\prime}, \mathbf{x}^{\prime}\right)\right|_{t=t^{\prime}}=i\left(\operatorname{ch} t^{\prime}\right)^{d-1} \delta\left(\mathbf{x}, \mathbf{x}^{\prime}\right) .
\end{aligned}
$$

\footnotetext{
${ }^{2}$ In the sequel we shall mostly ignore the $M$-dependence of the coefficients $a_{\lambda l M}$ and $b_{\lambda l M}$ in Eq. 3.16). This will enforce rotational invariance in the chosen frame.
} 
These requirements have, for each $\lambda$, a unique solution which is an entire function of $\lambda$ and is antisymmetric and Lorentz invariant in the variables $x, x^{\prime}$. To find it, we generalize the method of canonical quantization to fields with complex squared masses by defining an involution in the space of solutions of Eq. (3.11) as follows:

$$
\phi_{\lambda l M}(t, \mathbf{x}) \rightarrow \phi_{\lambda l M}^{*}(t, \mathbf{x})=(\operatorname{ch} t)^{-\kappa}\left[\bar{a}_{l} \mathbf{P}_{\lambda+\kappa}^{-l-\kappa}(-i \operatorname{sh} t)+\bar{b}_{l} \mathbf{P}_{\lambda+\kappa}^{-l-\kappa}(i \operatorname{sh} t)\right] Y_{l M}(\mathbf{x}) .
$$

where $a_{l}=a_{l}(\lambda)$ and $b_{l}=b_{l}(\lambda)$. When $t$ and $m_{\lambda}^{2}$ are real, the above involution coincides with the complex conjugation $\phi_{\lambda l M}^{*}(t, \mathbf{x})=\overline{\phi_{\lambda l M}(t, \mathbf{x})}$; this follows from Eq. (3.14) for $\lambda$ satisfying (3.3) while it is obvious for $\lambda$ real.

Notice that $\phi_{\lambda l M}^{*}(t, \mathbf{x})$ is also a solution of Eq. (3.11) (while the complex conjugate $\overline{\phi_{\lambda l M}(t, \mathbf{x})}$ in general is not; only when $m_{\lambda}^{2}$ is real). We are thus led to define a generalized Klein-Gordon product

$$
(f, g)_{K G}=i \int_{\Sigma}\left(f^{*} \partial_{\mu} g-g \partial_{\mu} f^{*}\right) d \Sigma^{\mu}(x)
$$

that coincides with the usual Klein-Gordon product (see e.g. 57, p. 49]) when $m_{\lambda}^{2}$ is real.

If $\lambda-l$ is not a non-negative integer and

$$
\left|a_{l}\right|^{2}-\left|b_{l}\right|^{2} \neq 0
$$

the solutions $\left\{\phi_{\lambda l M}(t, \mathbf{x}), \phi_{\lambda l M}^{*}(t, \mathbf{x})\right\}$ are well-defined and all together constitute a complete and orthogonal set w.r.t. the generalized Klein-Gordon product. This can be seen by using the following basic Wronskian relations

$$
\operatorname{Wr}\left(\mathbf{P}_{\nu}^{\mu}, \mathbf{Q}_{\nu}^{\mu}\right)=\frac{\Gamma(1+\nu+\mu)}{\Gamma(1+\nu-\mu)}\left(1-z^{2}\right)^{-1}
$$

which imply that

$$
\left(\phi_{\lambda l M}, \phi_{\lambda l^{\prime} M^{\prime}}\right)_{K G}=\frac{1}{\gamma_{l}}\left(\left|a_{l}\right|^{2}-\left|b_{l}\right|^{2}\right) \delta_{l l^{\prime}} \delta_{M M^{\prime}}, \quad\left(\phi_{\lambda l M}, \phi_{\lambda l^{\prime} M^{\prime}}^{*}\right)_{K G}=0,
$$

where we have defined

$$
\gamma_{l}(\lambda)=\frac{1}{2} \Gamma(l-\lambda) \Gamma(1+\lambda+l+2 \kappa) .
$$

All in all, the covariant commutator admits the following series expansion in spherical harmonics

$$
\begin{aligned}
& C_{\lambda}\left(x, x^{\prime}\right)=\sum_{l, M} \frac{\gamma_{l}}{\left|a_{l}\right|^{2}-\left|b_{l}\right|^{2}}\left[\phi_{\lambda l M}(t, \mathbf{x}) \phi_{\lambda l M}^{*}\left(t^{\prime}, \mathbf{x}^{\prime}\right)-\phi_{\lambda l M}\left(t^{\prime}, \mathbf{x}^{\prime}\right) \phi_{\lambda l M}^{*}(t, \mathbf{x})\right]= \\
& =\sum_{l} \frac{\gamma_{l}(\lambda)\left[\mathbf{P}_{\lambda+\kappa}^{-l-\kappa}(i \operatorname{sh} t) \mathbf{P}_{\lambda+\kappa}^{-l-\kappa}\left(-i \operatorname{sh} t^{\prime}\right)-\mathbf{P}_{\lambda+\kappa}^{-l-\kappa}\left(i \operatorname{sh} t^{\prime}\right) \mathbf{P}_{\lambda+\kappa}^{-l-\kappa}(-i \operatorname{sh} t)\right]}{(\operatorname{ch} t)^{\kappa}\left(\operatorname{ch} t^{\prime}\right)^{\kappa}} \sum_{M} Y_{l M}(\mathbf{x}) Y_{l M}\left(\mathbf{x}^{\prime}\right)
\end{aligned}
$$

which does not depend on the choice of $a_{l}$ and $b_{l}$ as expected. As mentioned above, the commutator is an entire function of the $\lambda$ variable. Indeed the poles of the coefficients $\gamma_{l}(\lambda)$ are exactly compensated by zeros of the time-dependent factors and a de Sitter invariant canonical commutator exists also for the integer values of the variable $\lambda$. Hence (3.27) holds for all complex $\lambda$.

\subsection{Two-point functions: massive case}

The canonical commutation relations and the Klein-Gordon equation uniquely determine the commutation rules (3.27). A quantization of the Klein-Gordon field is accomplished by finding a Hilbert space representation of the above commutation rules. This amounts [45] to finding a two-point function $F\left(x, x^{\prime}\right)$, interpreted as the "vacuum" expectation value of the field

$$
F\left(x, x^{\prime}\right)=\left\langle\Omega, \phi(x) \phi\left(x^{\prime}\right) \Omega\right\rangle,
$$

which solves the Klein-Gordon equation w.r.t. both $x$ and $x^{\prime}$. The antisymmetric part of $F\left(x, x^{\prime}\right)$ is uniquely determined by the commutator through the functional equation

$$
F\left(x, x^{\prime}\right)-F\left(x^{\prime}, x\right)=C\left(x, x^{\prime}\right)
$$


The two-point function must also satisfy the positive semi-definiteness condition [17, 18]

$$
\int F\left(x, x^{\prime}\right) \bar{f}(x) f\left(x^{\prime}\right) d x d x^{\prime} \geq 0 .
$$

The above conditions still leave a large degree of arbitrariness as regards the symmetric part of $F$.

Let us focus for the moment on the standard massive fields $m_{\lambda}^{2}>0$. In this case the coefficients $\gamma_{l}(\lambda)$ are all strictly positive:

$$
\gamma_{l}(\lambda)=\left\{\begin{array}{lll}
\frac{1}{2} \Gamma\left(\frac{d-1}{2}+l+i \rho\right) \Gamma\left(\frac{d-1}{2}+l-i \rho\right)>0, & \lambda=-\frac{d-1}{2}+i \rho, & \rho \in \mathbf{R}, \\
\frac{1}{2} \Gamma\left(\frac{d-1}{2}+l+\rho\right) \Gamma\left(\frac{d-1}{2}+l-\rho\right)>0, & \lambda=-\frac{d-1}{2}+\rho, \quad \rho \in \mathbf{R}, \quad|\rho|<\frac{d-1}{2} .
\end{array}\right.
$$

This property allows us to pose $a_{l}=\operatorname{ch} \alpha_{l}, b_{l}=e^{i \beta_{l}} \operatorname{sh} \alpha_{l}$ with $\alpha_{l}$ and $\beta_{l}$ real, and define the corresponding canonical orthonormal modes as follows:

$$
\psi_{\lambda l M}(t, \mathbf{x})=\sqrt{\gamma_{l}(\lambda)}(\operatorname{ch} t)^{-\kappa}\left[\operatorname{ch} \alpha_{l} \mathbf{P}_{\lambda+\kappa}^{-l-\kappa}(i \operatorname{sh} t)+e^{i \beta_{l}} \operatorname{sh} \alpha_{l} \mathbf{P}_{\lambda+\kappa}^{-l-\kappa}(-i \operatorname{sh} t)\right] Y_{l M}(\mathbf{x}) .
$$

The two-point function associated with the above choice of modes (3.32) through the standard Fock construction may be explicitly written as a series

$$
\begin{aligned}
F\left(x, x^{\prime}\right) & =\sum_{l M} \psi_{\lambda l M}(t, \mathbf{x}) \bar{\psi}_{\lambda l M}\left(t^{\prime}, \mathbf{x}^{\prime}\right) \\
& =\sum_{l M} \gamma_{l}(\lambda) \phi_{\lambda l M}(t, \mathbf{x}) \phi_{\lambda l M}^{*}\left(t^{\prime}, \mathbf{x}^{\prime}\right)
\end{aligned}
$$

and is manifestly positive-semi-definite [17, 18, because the coefficients (3.31) are all positive. Eq. (3.34) admits a further generalization that describes also mixed stated as follows:

$$
\begin{aligned}
F\left(x, x^{\prime}\right) & =\sum_{l} \gamma_{l}(\lambda)\left(\operatorname{ch} t \operatorname{ch} t^{\prime}\right)^{-\kappa}\left[\operatorname{ch}^{2} \alpha_{l} \mathbf{P}_{\lambda+\kappa}^{-l-\kappa}(i \operatorname{sh} t) \mathbf{P}_{\lambda+\kappa}^{-l-\kappa}\left(-i \operatorname{sh} t^{\prime}\right)+\right. \\
& +\operatorname{sh}^{2} \alpha_{l} \mathbf{P}_{\lambda+\kappa}^{-l-\kappa}(-i \operatorname{sh} t) \mathbf{P}_{\lambda+\kappa}^{-l-\kappa}\left(i \operatorname{sh} t^{\prime}\right)+\rho_{l} \operatorname{ch} \alpha_{l} \operatorname{sh} \alpha_{l} e^{-i \beta_{l}} \mathbf{P}_{\lambda+\kappa}^{-l-\kappa}(i \operatorname{sh} t) \mathbf{P}_{\lambda+\kappa}^{-l-\kappa}\left(i \operatorname{sh} t^{\prime}\right)+ \\
& \left.+\rho_{l} \operatorname{ch} \alpha_{l} \operatorname{sh} \alpha_{l} e^{i \beta_{l}} \mathbf{P}_{\lambda+\kappa}^{-l-\kappa}(-i \operatorname{sh} t) \mathbf{P}_{\lambda+\kappa}^{-l-\kappa}\left(-i \operatorname{sh} t^{\prime}\right)\right] \sum_{M} Y_{l M}(\mathbf{x}) Y_{l M}\left(\mathbf{x}^{\prime}\right)
\end{aligned}
$$

where $0 \leq \rho_{l} \leq 1$. The case $\rho_{l}=1$ coincides with the pure states 3.34).

\subsection{Into the tachyons}

While (3.33) makes sense as such only for $m_{\lambda}^{2}$ positive, Eqs. (3.34) and 3.35 are well defined also for complex values of the $\lambda$ variable; furthermore, if $\alpha_{l}(\lambda), \beta_{l}(\lambda)$ and $\rho_{l}(\lambda)$ are well-behaved, the series converge and define meromorphic functions of $\lambda$ with poles at the integers.

Focusing on the pure states (3.34) we see in particular that when $\lambda \rightarrow 0$ from the left the coefficient $\gamma_{0}(\lambda)$ explodes; then it becomes negative in the interval $0<\lambda<1$, diverging to minus infinity at the endpoints (see the figure). Therefore, in the interval $0<\lambda<1$ the modes $\phi_{\lambda 00}$ have negative norm. Similarly, when $\lambda \rightarrow n$ the coefficients $\gamma_{0}(\lambda), \gamma_{1}(\lambda), \ldots, \gamma_{n}(\lambda)$ diverge. In the interval $n<\lambda<n+1$ all the $\gamma_{l}(\lambda)$ such that $n-l$ is even are negative and the corresponding modes $\phi_{\lambda l M}$ have negative norms. The advantage of using the spherical coordinates is that the infrared problem relative to the tachyons manifests itself by altering the behaviour of a finite number of modes, because of the compactness of the spatial sections.

Therefore, if $\alpha_{l}(\lambda), \beta_{l}(\lambda)$ and $\rho_{l}(\lambda)$ are well-behaved, the conclusion is that there is no issue in the convergence of the series Eqs. (3.34) and 3.35) or of the series for the corresponding Green functions [12, 15], but the two-point functions defined this way are not positive semi-definite (see [46], footnote 1). This is true in particular for the choice $\alpha_{l}=\beta_{l}=0, \rho_{l}=1$ corresponding to the maximally analytic invariant (Bunch-Davies) vacuum (see Eq. (4.28) below).

We will see that, while in general it is necessary to fully break the de Sitter symmetry to reestablish a positive quantization, it is precisely the divergence of the coefficients $\gamma_{l}(n)$ that allows to construct de Sitter invariant quantizations of the tachyon fields for the discrete values of the masses $m_{n}^{2}$. The non invariant case will be treated in Section 8 at the end of the paper where we will describe all possible non-invariant canonical quantizations with positive metric. 

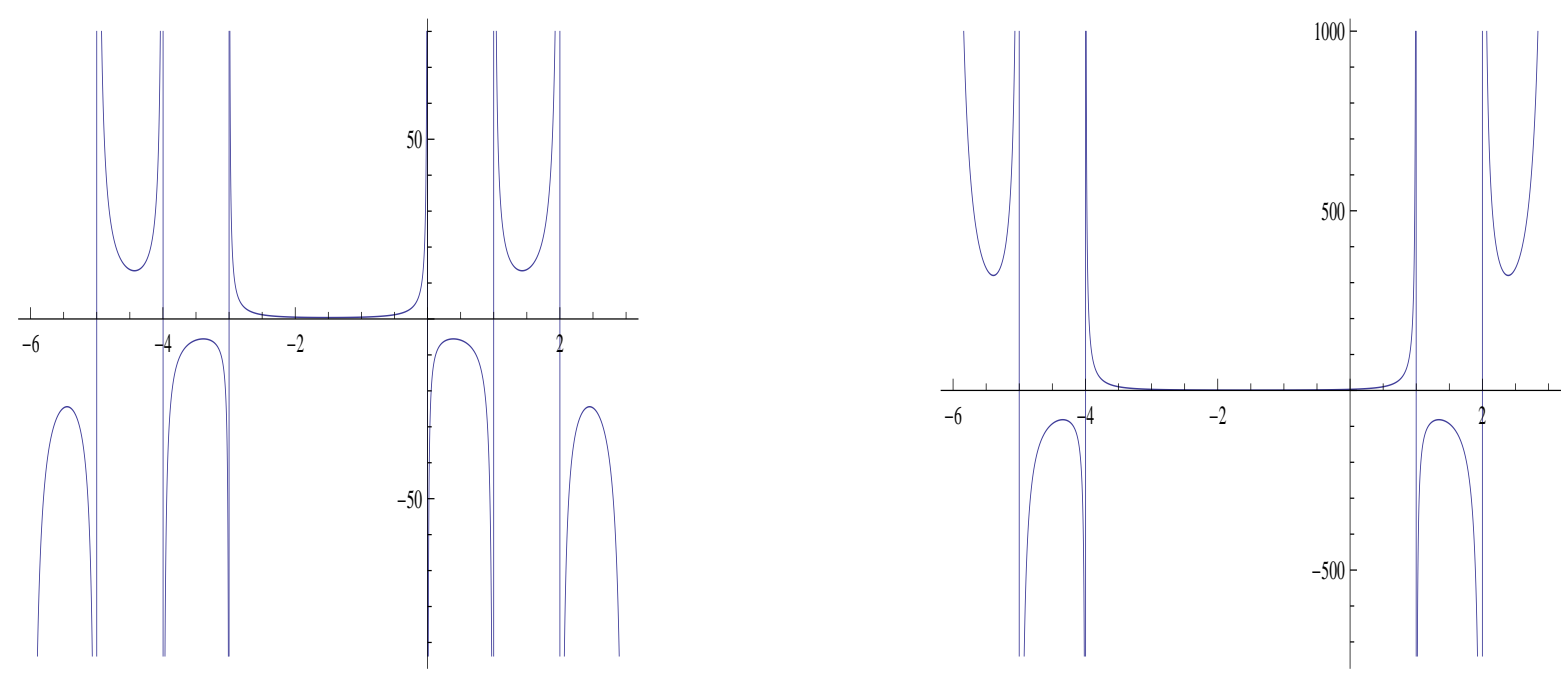

Figure 1: Plot of $\gamma_{0}(\lambda)$ (left) and $\gamma_{1}(\lambda)$ (right) in the four-dimensional case $d=4$.

\section{Plane waves and de Sitter invariant two-point func- tions}

\subsection{Holomorphic plane waves}

Even though Eq. (3.35) provides through the standard reconstruction procedure 17 the most general class of Fock canonical quantizations of the de Sitter Klein-Gordon massive free field, we are interested in singling out among them the de Sitter invariant vacua. To this aim we make appeal to a remarkable set of solutions of the de Sitter Klein Gordon equation which may be interpreted as de Sitter plane waves 28, 47, 30. Here is their definition. For a given nonzero lightlike vector $\xi \in C_{+}$and a complex number $\lambda \in \mathbf{C}$ let us construct the homogeneous function

$$
z \mapsto(\xi \cdot z)^{\lambda} .
$$

When considered in the ambient Minkowski spacetime this is a holomorphic function in the tubes $T_{ \pm}$and satisfies the massless Klein-Gordon equation $\square_{\text {Mink }, z}(\xi \cdot z)^{\lambda}=0$. When restricted to the de Sitter universe it is holomorphic in the tuboids $\mathcal{T}_{ \pm}$and satisfies the massive (complex) Klein-Gordon equation:

$$
\left(\square_{z}+m_{\lambda}^{2}\right)(\xi \cdot z)^{\lambda}=0 .
$$

The parameter $\lambda$ is here unrestricted, i.e. we consider complex squared masses $m_{\lambda}^{2}$. The symmetry (2.25) also implies that, corresponding to the same complex squared mass,

$$
\left(\square_{z}+m_{\lambda}^{2}\right)(\xi \cdot z)^{-\lambda-d+1}=0 .
$$

The boundary values

$$
(\xi \cdot x)_{ \pm}^{\lambda}=\lim _{z \in T_{ \pm}, z \rightarrow x}(\xi \cdot z)^{\lambda},
$$

are homogeneous distributions of degree $\lambda$ on $M_{d+1}$, and their restrictions to $X_{d}$, denoted with the same symbols, are solutions of the de Sitter Klein-Gordon equation

$$
\left(\square_{x}+m_{\lambda}^{2}\right)(\xi \cdot x)_{ \pm}^{\lambda}=0, \quad\left(\square_{x}+m_{\lambda}^{2}\right)(\xi \cdot x)_{ \pm}^{1-d-\lambda}=0 .
$$

All these objects depend in a $\mathcal{C}^{\infty}$ way on $\xi$ and are entire in $\lambda$.

For $f \in \mathcal{D}\left(X_{d}\right), \xi \in \mathcal{C}_{+} \backslash\{0\}, \lambda \in \mathbf{C}$, the holomrphic plane waves may be used to define the 
Fourier transforms as follows [28, 48]:

$$
\begin{aligned}
& \tilde{f}_{+}(\xi, \lambda)=\tilde{f}_{\lambda,+}(\xi)=\int_{X_{d}}(\xi \cdot x)_{+}^{\lambda} f(x) d x \\
& \tilde{f}_{-}(\xi, \lambda)=\tilde{f}_{\lambda,-}(\xi)=\int_{X_{d}}(\xi \cdot x)_{-}^{\lambda} f(x) d x .
\end{aligned}
$$

These definitions obviously commute with the Lorentz transformations.

The following remarkable property, which will be used later, shows the relationship between the waves (4.1) and (4.3):

Lemma 4.1 If $\operatorname{Re} \lambda>-(d-1) / 2$ and $z \in \mathcal{T}_{ \pm}$, then

$$
(\xi \cdot z)^{\lambda}=\frac{e^{ \pm i \pi\left(\lambda+\frac{d-1}{2}\right)} \Gamma(\lambda+d-1)}{\pi^{\frac{d-1}{2}} 2^{\lambda+d-1} \Gamma\left(\lambda+\frac{d-1}{2}\right)} \int_{\gamma}\left(\xi \cdot \xi^{\prime}\right)^{\lambda}\left(\xi^{\prime} \cdot z\right)^{1-d-\lambda} \alpha\left(\xi^{\prime}\right),
$$

where $\gamma$ is any smooth $(d-1)$-cycle homotopic to the sphere $S_{0}=\left\{\xi \in C_{+}: \xi^{0}=1\right\}$ in $C_{+} \backslash\{0\}$ and $\alpha$ is the $(d-1)$-differential form

$$
\alpha(\xi)=\left(\xi^{0}\right)^{-1} \sum_{j=1}^{d}(-1)^{j+1} \xi^{j} d \xi^{1} \wedge \ldots \widehat{d \xi^{j}} \wedge \ldots d \xi^{d} .
$$

Proof: on $C_{+} \backslash\{0\}, \xi^{0}=\sqrt{\sum_{j=1}^{d}\left(\xi^{j}\right)^{2}}$ and if $\varphi$ is a smooth function on $C_{+} \backslash\{0\}$,

$$
d(\varphi(\xi) \alpha(\xi))=\left((d-1) \varphi(\xi)+\sum_{j=1}^{d} \xi^{j} \frac{\partial \varphi(\xi)}{\partial \xi^{j}}\right)\left(\xi^{0}\right)^{-1} d \xi^{1} \wedge \ldots \wedge d \xi^{d}
$$

If $\varphi$ is homogeneous of degree $(1-d)$ the form $\varphi \alpha$ is closed. For any smooth $(d-1)$-cycle $\gamma$ homotopic to $S_{0}$ in $C_{+} \backslash\{0\}$ one has that

$$
(\mathrm{D}+d-1) \varphi=0 \Longrightarrow \int_{S_{0}} \varphi(\xi) d \boldsymbol{\xi}=\int_{\gamma} \varphi(\xi) \alpha(\xi)
$$

where $d \boldsymbol{\xi}$ is the standard measure on the sphere $S_{0}$. In the same case the above integral is invariant under Lorentz transformations, i.e. for any $\Lambda \in L_{+}^{\uparrow}$

$$
\int_{S_{0}} \varphi(\xi) d \boldsymbol{\xi}=\int_{S_{0}} \varphi\left(\Lambda^{-1} \xi\right) d \boldsymbol{\xi}
$$

Now, since the integral (4.8) is convergent, it follows from the analyticity, invariance and homogeneity of the rhs that the two sides of (4.8) are equal up to a constant factor. The constant can be evaluated by choosing $z=(0, \ldots, 0, \pm i), \xi=(1,0, \ldots, 0,1)$. With this choice,

$$
\begin{aligned}
\int_{S_{0}}\left(\xi \cdot \xi^{\prime}\right)^{\lambda}\left(\xi^{\prime} \cdot z\right)^{1-d-\lambda} d \xi^{\prime} & =\frac{2 \pi^{\frac{d-1}{2}} e^{ \pm \frac{i \pi}{2}(1-d-\lambda)}}{\Gamma\left(\frac{d-1}{2}\right)} \int_{0}^{\pi}(1-\cos \theta)^{\lambda}(\sin \theta)^{d-2} d \theta \\
& =\pi^{\frac{d-1}{2}} e^{ \pm \frac{i \pi}{2}(1-d-\lambda)} \frac{2^{\lambda+d-1} \Gamma\left(\lambda+\frac{d-1}{2}\right)}{\Gamma(\lambda+d-1)},
\end{aligned}
$$

and the assertion of the lemma follows.

\subsection{Maximally analytic vacua}

We will need a small part of the harmonic analysis on the de Sitter space (see e.g. 28]). The main result is the following: for $z \in \mathcal{T}_{-}$and $z^{\prime} \in \mathcal{T}_{+}$one has

$$
\begin{aligned}
& \int_{S_{0}}(\xi \cdot z)^{1-d-\lambda}\left(\xi \cdot z^{\prime}\right)^{\lambda} d \boldsymbol{\xi}=\int_{\gamma}(\xi \cdot z)^{1-d-\lambda}\left(\xi \cdot z^{\prime}\right)^{\lambda} \alpha(\xi)= \\
& =\frac{2 \pi^{\frac{d}{2}} e^{i \pi\left(\lambda+\frac{d-1}{2}\right)}}{\Gamma\left(\frac{d}{2}\right)} F\left(-\lambda, \lambda+d-1 ; \frac{d}{2} ; \frac{1-\zeta}{2}\right), \quad \zeta=z \cdot z^{\prime}, \quad \lambda \in \mathbf{C} .
\end{aligned}
$$


Here as above $S_{0}$ is identical to the unit sphere in $\mathbf{R}^{d}$ and $\gamma$ is any smooth $(d-1)$-cycle homotopic to $S_{0}$ in $C_{+} \backslash\{0\}$ and $\alpha$ is the $(d-1)$-differential form (4.9). The equality in (4.14) is proven along the lines of the proof of the previous lemma. Lorentz invariance is then used to compute (4.15). Both sides of (4.14) are entire in $\lambda \in \mathbf{C}$, as well as the rhs of (4.15).

If $\lambda$ is not a pole of $\Gamma(-\lambda) \Gamma(\lambda+d-1), z \in \mathcal{T}_{-}, z^{\prime} \in \mathcal{T}_{+}$, we denote

$$
\begin{aligned}
W_{\lambda}\left(z, z^{\prime}\right) & =w_{\lambda}\left(z \cdot z^{\prime}\right)=c(\lambda) \int_{S_{0}}(\xi \cdot z)^{1-d-\lambda}\left(\xi \cdot z^{\prime}\right)^{\lambda} d \boldsymbol{\xi} \\
c(\lambda) & =\frac{\Gamma(-\lambda) \Gamma(\lambda+d-1) e^{-i \pi\left(\lambda+\frac{d-1}{2}\right)}}{2^{d+1} \pi^{d}}
\end{aligned}
$$

We list three equivalent expressions for $w_{\lambda}$ :

$$
\begin{aligned}
w_{\lambda}\left(z \cdot z^{\prime}\right) & =\frac{\Gamma(-\lambda) \Gamma(\lambda+d-1)}{(4 \pi)^{d / 2} \Gamma\left(\frac{d}{2}\right)} F\left(-\lambda, \lambda+d-1 ; \frac{d}{2} ; \frac{1-\zeta}{2}\right) \\
& =\frac{\Gamma(-\lambda) \Gamma(\lambda+d-1)}{(4 \pi)^{d / 2} \Gamma\left(\frac{d}{2}\right)}\left(\frac{\zeta+1}{2}\right)^{-\frac{d-2}{2}} F\left(\lambda+\frac{d}{2},-\lambda-\frac{d-2}{2} ; \frac{d}{2} ; \frac{1-\zeta}{2}\right) \\
& =\frac{\Gamma(-\lambda) \Gamma(\lambda+d-1)}{2(2 \pi)^{d / 2}}\left(\zeta^{2}-1\right)^{-\frac{d-2}{4}} P_{\lambda+\frac{d-2}{2}}^{-\frac{d-2}{2}}(\zeta), \quad \zeta=z \cdot z^{\prime} .
\end{aligned}
$$

Eq. (4.19) follows from (4.18) by a standard identity between hypergeometric functions: 42, 2.1.4 (23) p. 64]. Here and in the sequel $z^{\alpha}=\exp (\alpha \log z)$ is defined as holomorphic in $\mathbf{C} \backslash \mathbf{R}_{-}$and $\left(z^{2}-1\right)^{\alpha}$ as $z^{2 \alpha}\left(1-z^{-2}\right)^{\alpha}$. The function $z \mapsto\left(1-z^{-2}\right)^{\alpha}$ is holomorphic and even in $\mathbf{C} \backslash[-1,1]$. The function $z \mapsto\left(z^{2}-1\right)^{\alpha}$ is holomorphic in

$$
\Delta_{1}=\mathbf{C} \backslash(-\infty, 1],
$$

and it is not even unless $\alpha$ is an integer. $z \mapsto\left(1-z^{2}\right)^{\alpha}$ is holomorphic and even in the domain $\Delta_{2}$ (see (3.13) ). It satisfies

$$
\left(1-z^{2}\right)^{\alpha}=e^{\mp i \pi \alpha}\left(z^{2}-1\right)^{\alpha} \quad \text { for } \quad \pm \operatorname{Im} z>0 .
$$

It is clear from (4.18) that $\zeta \mapsto w_{\lambda}(\zeta)=w_{-\lambda-d+1}(\zeta)$ is holomorphic in $\mathbf{C} \backslash(-\infty,-1]$ i.e. everywhere except on the locality cut (maximal analyticity property).

Recall that the distribution $(\xi \cdot x)_{ \pm}^{\lambda}$ on $X_{d}$ depends in a $\mathcal{C}^{\infty}$ way on $\xi \in C_{+} \backslash\{0\}$, and is entire in $\lambda$. The function $W_{\lambda}\left(z, z^{\prime}\right)$ has a boundary value $\mathcal{W}_{\lambda}\left(x, x^{\prime}\right)$ in the sense of distributions as $z$ and $z^{\prime}$ tend to the reals from $\mathcal{T}_{-}$and $\mathcal{T}_{+}$, respectively, and

$$
\mathcal{W}_{\lambda}\left(x, x^{\prime}\right)=c(\lambda) \int_{S_{0}}(\xi \cdot x)_{-}^{1-d-\lambda}\left(\xi \cdot x^{\prime}\right)_{+}^{\lambda} d \boldsymbol{\xi} .
$$

Since $(\xi \cdot x)_{ \pm}^{\lambda}$ satisfies the Klein-Gordon equation [4.5), $W_{\lambda}$ and $\mathcal{W}_{\lambda}$ satisfy the same equation in each of their arguments. Furthermore

$$
\mathcal{W}_{\lambda}\left(x, x^{\prime}\right)=\mathcal{W}_{\lambda}\left(-x^{\prime},-x\right)=\overline{\mathcal{W}_{\bar{\lambda}}\left(x^{\prime}, x\right)}
$$

(provided $\lambda$ is not a pole of $\Gamma(-\lambda) \Gamma(\lambda+d-1)$ ).

Eq. (4.16) and its boundary value (4.23) are Fourier-type representation of the so called BunchDavies aka Euclidean two-point function, which occupies such a considerable portion of the de Sitter quantum field theoretical literature. Its role and relevance for understanding de Sitter physics is still under debate [49, 50, 51, 52, 53.

\subsection{Invariant vacua in spherical coordinates}

To establish the connection between the above manifestly invariant expressions for the two-point functions, the modes (3.32) and the two-point functions (3.34) let again $x=x(t, \mathbf{x})$ be parameterized as in Eq. (2.7), with $\mathrm{x}$ real. For $0<\operatorname{Im} t<\pi$ the event $x$ belongs to forward tube $\mathcal{T}_{+}$while 
for $-\pi<\operatorname{Im} t<0$ it belongs to the past tube $\mathcal{T}_{-}$; for such events the following fundamental representation holds (see appendix B):

$$
\begin{array}{r}
(\xi \cdot x)^{\lambda}=2 \pi\left(\frac{2 \pi}{\operatorname{ch} t}\right)^{\kappa} \sum_{l=0}^{\infty} e^{ \pm \frac{i \pi}{2}(\lambda-l)} \frac{\Gamma(l-\lambda)}{\Gamma(-\lambda)} \mathbf{P}_{\lambda+\kappa}^{-l-\kappa}(\mp i \operatorname{sh} t) \sum_{M} Y_{l M}(\boldsymbol{\xi}) Y_{l M}(\mathbf{x}) \\
\text { for } x=x(t, \mathbf{x}) \text { with } 0< \pm \operatorname{Im} t<\pi, \quad \xi \in S_{0} .
\end{array}
$$

Eq. (4.25) gives the projection of the holomorphic plane waves onto the hyperspherical harmonics:

$$
\Xi_{\lambda l M}(x)=\frac{(-1)^{l} \Gamma(\lambda+1-l)}{(2 \pi)^{\frac{d}{2}} \Gamma(\lambda+1)} \int_{S_{0}}(\xi \cdot x)^{\lambda} Y_{l M}(\boldsymbol{\xi}) d \boldsymbol{\xi}=h_{\lambda l}(t) Y_{l M}(\mathbf{x})
$$

where $x=x(t, \mathbf{x})$ and $h_{\lambda l}(t)$ is given by

$$
h_{\lambda l}(t)= \begin{cases}e^{\frac{i \pi}{2}(\lambda-l)}(\operatorname{ch} t)^{-\kappa} \mathbf{P}_{\lambda+\kappa}^{-l-\kappa}(-i \operatorname{sh} t), & 0<\operatorname{Im} t<\pi, \\ e^{-\frac{i \pi}{2}(\lambda-l)}(\operatorname{ch} t)^{-\kappa} \mathbf{P}_{\lambda+\kappa}^{-l-\kappa}(i \operatorname{sh} t), & -\pi<\operatorname{Im} t<0 .\end{cases}
$$

These formulae define $\Xi_{\lambda l M}$ as a holomorphic function on $\mathcal{T}_{+} \cup \mathcal{T}_{-}$which has two boundary values on the reals from these two tuboids. Eqs. (4.27) show that these boundary values are actually continuous. They are not equal unless $\lambda$ is an integer $\geq l$ (see sect. 6). Which of the two boundary values is used will be clear from the context. Note that $\Xi_{\lambda l M}$ is entire in $\lambda$ and that for real $\lambda$, $\Xi_{\lambda l M}(x)=\overline{\Xi_{\lambda l M}(\bar{x})}$.

The remarkable fact is that the plane waves holomorphic in $\mathcal{T}_{ \pm}$contain, respectively, only the pure modes $\mathbf{P}_{\lambda+\kappa}^{-l-\kappa}(\mp i \operatorname{sh} t)$ and not a linear combination of them, as it happens in general for a solution of the de Sitter Klein-Gordon equation. Inserting the representation (4.25) into (4.16) and taking into account the identity $\mathbf{P}_{\nu}^{\mu}=\mathbf{P}_{-\nu-1}^{\mu}$ we get

$$
\begin{aligned}
\mathcal{W}_{\lambda}\left(x, x^{\prime}\right) & =\frac{1}{(\operatorname{ch} t)^{\kappa}\left(\operatorname{ch} t^{\prime}\right)^{\kappa}} \sum_{l} \gamma_{l}(\lambda) \mathbf{P}_{\lambda+\kappa}^{-l-\kappa}(i \operatorname{sh} t) \mathbf{P}_{\lambda+\kappa}^{-l-\kappa}\left(-i \operatorname{sh} t^{\prime}\right) \sum_{M} Y_{l M}(\mathbf{x}) Y_{l M}\left(\mathbf{x}^{\prime}\right) \\
& =\sum_{l, M} \gamma_{l}(\lambda) \Xi_{\lambda l M}(x) \Xi_{\lambda l M}\left(x^{\prime}\right)
\end{aligned}
$$

The Bunch-Davies two-point function (4.23) therefore coincides with the simplest conceivable choice in Eq. (3.34): $\alpha_{l}=\beta_{l}=0$. The choice $\alpha_{l}=\alpha, \beta_{l}=\pi l$ corresponds instead to the so-called alphavacua. Indeed, since $Y_{l M}(-\mathbf{x})=e^{i \pi l} Y_{l M}(\mathbf{x})$ we get

$$
F\left(x, x^{\prime}\right)=\operatorname{ch}^{2} \alpha \mathcal{W}_{\lambda}\left(x, x^{\prime}\right)+\operatorname{sh}^{2} \alpha \mathcal{W}_{\lambda}\left(x^{\prime}, x\right)+\operatorname{ch} \alpha \operatorname{sh} \alpha\left[\mathcal{W}_{\lambda}\left(x,-x^{\prime}\right)+\mathcal{W}_{\lambda}\left(-x, x^{\prime}\right)\right]
$$

More generally, by setting $\alpha_{l}=\alpha, \beta_{l}=\pi l+\beta$ we obtain the most general de Sitter invariant vacua first described in [35] and studied in [38, 39] and by several authors afterwards. Once more, the commutator $C_{\lambda}\left(x, x^{\prime}\right)$ does not depend on which choice one makes.

\section{Massless fields and tachyons}

Let $n$ denote a fixed non-negative integer. We saw in Section (3.3) the normalization of the modes with angular momentum up to $l=n$ diverges in the limit $\lambda \rightarrow n$ for almost all choices of the constants $\alpha_{l}$ and $\beta_{l}$. Correspondingly, all the plane waves $(\xi \cdot x)^{n}$ are non-normalizable zero modes (see Eq. 4.25). The point is that such waves are polynomials of degree $n$, globally defined and analytic in the complex de Sitter manifold - actually in the whole complex ambient spacetime. The space of such polynomials is finite dimensional and, as such, not rich enough to reconstruct the infinitely many degrees of freedom of a quantum field.

The aforementioned divergence has a counterpart in the presence of the factor $\Gamma(-\lambda)$ in the normalization of the two-point function $\mathcal{W}_{\lambda}\left(x, x^{\prime}\right)$ as given in Eq. (4.18); as a function of $\lambda \mathcal{W}_{\lambda}\left(x, x^{\prime}\right)$ has a pole at every nonnegative integer $n$, the case $n=0$ being the massless minimally coupled field. If this factor is simply removed, the resulting two-point function tends to a polynomial in $x \cdot x^{\prime}$ which as such has no quantum feature. On the other hand, we saw that the commutator function 
$C_{\lambda}\left(x, x^{\prime}\right)$ associated to $\mathcal{W}_{\lambda}\left(x, x^{\prime}\right)$ does not have any pole at $n$; it is an entire function of $\lambda$ and removing the factor $\Gamma(-\lambda)$ would spoil its good behavior at $n$. The fact that the unitary irreducible representations of the Lorentz group of the discrete series exist precisely for squared masses of the form $m_{n}^{2}=-n(n+d-1)$ suggests that in spite of, and actually thanks to that divergence, an acceptable quantum field theory might exist for such squared masses.

In this and the two following Sections we provide a construction of such theories with explicit Lorentz invariance analogous to the Gupta-Bleuler quantization of QED.

\subsection{Regularized two point function at $\lambda=n$. Equation of motion}

Suppose $\operatorname{Re} \lambda+d-1>0$ and let

$$
\begin{aligned}
& G_{\lambda}\left(x, x^{\prime}\right) \stackrel{\text { def }}{=} \frac{1}{\Gamma(-\lambda)} w_{\lambda}\left(x \cdot x^{\prime}\right)=\sum_{l=0}^{\infty} A_{1}(\lambda, l) \sum_{M} \Xi_{\lambda l M}(x) \Xi_{\lambda l M}\left(x^{\prime}\right) \\
& A_{1}(\lambda, l)=\frac{(-1)^{l} \Gamma(l+\lambda+d-1) \Gamma(\lambda+1)}{2 \Gamma(\lambda+1-l)}
\end{aligned}
$$

The identity at the rhs of (5.1) is valid for $0<-\operatorname{Im} t<\pi, 0<\operatorname{Im} t^{\prime}<\pi$. When $\lambda=n$ the sum over $l$ in Eq. (5.1) stops at $l=n$. Eq. (4.18) then implies that

$G_{n}\left(x, x^{\prime}\right)=\frac{\Gamma(n+d-1)}{(4 \pi)^{d / 2}} F\left(-n, n+d-1 ; \frac{d}{2} ; \frac{1-\zeta}{2}\right)=\frac{\Gamma(n+1) \Gamma(d-1)}{(4 \pi)^{d / 2}} C_{n}^{\frac{d-1}{2}}(\zeta), \zeta=x \cdot x^{\prime}$.

$C_{n}^{\frac{d-1}{2}}$ is a Gegenbauer polynomial of degree $n$ (see Appendix $\mathrm{A}$ ) so that $G_{n}\left(x, x^{\prime}\right.$ ) is a polynomial of degree $n$ with real coefficients in $x \cdot x^{\prime}$. Eq. (4.16) also implies that

$$
G_{n}\left(z, z^{\prime}\right)=\frac{\Gamma(n+d-1) e^{-i \pi\left(n+\frac{d-1}{2}\right)}}{2^{d+1} \pi^{d}} \int_{S_{0}}(\xi \cdot z)^{1-d-n}\left(\xi \cdot z^{\prime}\right)^{n} d \boldsymbol{\xi}, \quad z \in \mathcal{T}_{-} .
$$

For $\lambda \sim n, \Gamma(-\lambda) \sim(-1)^{n+1} / n !(\lambda-n)$. We can attempt to remove the pole of $w_{\lambda}$ at $n$ by defining

$$
\begin{aligned}
\widehat{W}_{n}\left(z, z^{\prime}\right)=\widehat{w}_{n}\left(z \cdot z^{\prime}\right) & =\left.\Gamma(-\lambda)\left(G_{\lambda}\left(z, z^{\prime}\right)-G_{n}\left(z, z^{\prime}\right)\right)\right|_{\lambda=n} \\
& =\left.\frac{(-1)^{n+1}}{n !} \frac{d}{d \lambda} G_{\lambda}\left(z, z^{\prime}\right)\right|_{\lambda=n} .
\end{aligned}
$$

The boundary values $\widehat{\mathcal{W}}_{n}\left(x, x^{\prime}\right)$ are similarly defined. The regularization that we have just used can be applied to any function $f(\lambda)$ which has at worst a simple pole at $\lambda=n$, i.e. we can define

$$
\widehat{f}(n)=\left.\frac{(-1)^{n+1}}{n !} \frac{d}{d \lambda}\left[\frac{f(\lambda)}{\Gamma(-\lambda)}\right]\right|_{\lambda=n}
$$

If $f$ is regular at $n$, the procedure leaves it unchanged, while if $f(\lambda)=a(\lambda-n)^{-1}+h(\lambda)$, where $h$ is analytic at $n$, this gives

$$
\widehat{f}(n)=a \psi(1+n)+h(n) .
$$

Since $G_{n}\left(x, x^{\prime}\right)$ is a polynomial and is symmetric in $x$ and $x^{\prime}$, the commutator function $C_{\lambda}\left(x, x^{\prime}\right)$ associated to $\mathcal{W}_{\lambda}\left(x, x^{\prime}\right)$ (see (3.27) ) is, as already announced, holomorphic in $\lambda$ near $n$ and

$$
\widehat{\mathcal{W}}_{n}\left(x, x^{\prime}\right)-\widehat{\mathcal{W}}_{n}\left(x^{\prime}, x\right)=C_{n}\left(x, x^{\prime}\right)=\left.C_{\lambda}\left(x, x^{\prime}\right)\right|_{\lambda=n},
$$

i.e. it needs no subtraction. In particular the canonical equal-time commutation equations 3.20) remain valid for $\lambda=n$. Furthermore the commutator solves the right Klein-Gordon equation

$$
\left(\square_{x, x^{\prime}}-n(n+d-1)\right) C_{n}\left(x, x^{\prime}\right)=0 .
$$


$\widehat{W}_{n}\left(z, z^{\prime}\right)$ has the same good properties of analyticity and invariance as $W_{\lambda}\left(z, z^{\prime}\right)$; on the other hand the regularization procedure has introduced an anomaly: the two-point function now satisfies an inhomogeneous Klein-Gordon equation

$$
\left(\square_{z, z^{\prime}}-n(n+d-1)\right) \widehat{W}_{n}\left(z, z^{\prime}\right)=\frac{(-1)^{n+1}}{n !}(2 n+d-1) G_{n}\left(z, z^{\prime}\right) .
$$

The pole removing procedure of (5.5) is, of course, not unique, since any function of $\lambda$ with a simple pole at $n$ could have been used instead of $\Gamma(-\lambda)$. The result would differ from $\widehat{W}_{n}\left(z, z^{\prime}\right)$ by a multiple of $G_{n}\left(z, z^{\prime}\right)$, without affecting (5.8) or (5.10).

\section{6 de Sitter invariant quantization of the tachyons}

Recall that the sesquilinear form defined on the space $\mathcal{D}\left(X_{d}\right)$ of test-functions on $X_{d}$

$$
\langle f, g\rangle_{\lambda}=\int_{X_{d} \times X_{d}} \overline{f(x)} \mathcal{W}_{\lambda}\left(x, x^{\prime}\right) g\left(x^{\prime}\right) d x d x^{\prime}
$$

is not positive semi-definite when $\lambda$ belong to the complement of the union of the sets 3.3 and (3.4). In particular (6.1) is not positive semi-definite when $\lambda$ is real and positive (and of course not integer). Furthermore, there exists no Lorentz invariant subspace of $\mathcal{D}\left(X_{d}\right)$ where $\mathcal{W}_{\lambda}\left(x, x^{\prime}\right)$ is positive semi-definite.

The sesquilinear form

$$
\langle f, g\rangle=\int_{X_{d} \times X_{d}} \overline{f(x)} \widehat{\mathcal{W}}_{n}\left(x, x^{\prime}\right) g\left(x^{\prime}\right) d x d x^{\prime}
$$

also fails to be positive-semi-definite. It is remarkable that, as we will show below, $\widehat{\mathcal{W}}_{n}\left(x, x^{\prime}\right)$ is positive-semi-definite when restricted to the Lorentz invariant subspace $\mathcal{E}_{n}$ of $\mathcal{D}\left(X_{d}\right)$ defined as follows

Definition 6.1

$$
\begin{aligned}
\mathcal{E}_{n} & =\left\{f \in \mathcal{D}\left(X_{d}\right): \tilde{f}_{n}(\xi)=\int_{X_{d}} f(x)(\xi \cdot x)^{n} d x=0 \quad \forall \xi \in C_{+}\right\} \\
& =\left\{f \in \mathcal{D}\left(X_{d}\right): \int_{\gamma} \tilde{f}_{1-d-n,+}(\xi)\left(\xi \cdot \xi^{\prime}\right)^{n} \alpha(\xi)=0 \quad \forall \xi^{\prime} \in C_{+}\right\} .
\end{aligned}
$$

Here we have used the definitions (4.6), 4.7] and Lemma 4.1 Note that $\widetilde{f}_{n}=\widetilde{f}_{n,+}=\widetilde{f}_{n,-}$.

Eqs. 4.25.4.27 provide the following representation:

$$
(\xi \cdot x)^{n}=(2 \pi)^{\frac{d}{2}} \sum_{l=0}^{n} \frac{(-1)^{l} \Gamma(n+1)}{\Gamma(n+1-l)} \sum_{M} \Xi_{n l M}(x) Y_{l M}(\boldsymbol{\xi}),
$$

The lhs of this equation is a real pseudo-harmonic polynomial in $x$, hence so is (for $l \leq n$ )

$$
\Xi_{n l M}(x)=\frac{(-1)^{l} \Gamma(n+1-l)}{(2 \pi)^{\frac{d}{2}} \Gamma(n+1)} \int_{S_{0}}(\xi \cdot x)^{n} Y_{l M}(\boldsymbol{\xi}) d \boldsymbol{\xi} .
$$

The vector space space spanned by all polynomials $x \mapsto(\xi \cdot x)^{n}$ as $\xi$ runs over $S_{0}$ (or over $C_{+}$) is a Lorentz invariant subspace of the space $\underline{\mathcal{H}^{d+1, n}}$ of homogeneous pseudo-harmonic polynomials of degree $n$ on $M_{d+1}$, which carries an irreducible representation of the Lorentz group, so that these two spaces coincide. The space $\mathcal{E}_{n}$ is thus the orthogonal subspace in $\mathcal{D}\left(X_{d}\right)$ to $\underline{\mathcal{H}^{d+1, n}} \subset \mathcal{D}^{\prime}\left(X_{d}\right)$. The space $\mathcal{E}_{n}$ can be further characterized as follows:

Lemma 6.1 A function $\varphi \in \mathcal{D}\left(X_{d}\right)$ belongs to $\mathcal{E}_{n}$ if and only if it satisfies one of the following conditions:

(i) for all integer $l \in[0, n]$ and for all $M$

$$
\int_{X_{d}} \Xi_{n l M}(x) \varphi(x) d x=0 .
$$


(ii) For all $x \in X_{d}$,

$$
\int_{X_{d}} G_{n}\left(x, x^{\prime}\right) \varphi\left(x^{\prime}\right) d x^{\prime}=0 .
$$

Furthermore

$$
\begin{gathered}
\left(\square+m_{n}^{2}\right) \varphi \in \mathcal{E}_{n} \quad \forall \varphi \in \mathcal{D}\left(X_{d}\right), \\
\varphi \in \mathcal{D}\left(X_{d}\right), \quad \varphi(-x)=(-1)^{n+1} \varphi(x) \Longrightarrow \varphi \in \mathcal{E}_{n} .
\end{gathered}
$$

Part (i) follows from (6.5) and (6.6). If $\varphi \in \mathcal{E}_{n}$, it satisfies (ii) by (5.4). Conversely suppose that $\varphi$ satisfies (ii). Then by (5.4), for all $z \in \mathcal{T}_{-}$,

$$
\int_{S_{0}}(\xi \cdot z)^{1-d-n} \widetilde{\varphi}_{n}(\xi) d \boldsymbol{\xi}=0 .
$$

This implies $\widetilde{\varphi}_{n}=0$, i.e. $\varphi \in \mathcal{E}_{n}$ by virtue of Lemma C.2 proved in Appendix C The last assertions of the lemma follows from the characterization (6.3) of $\mathcal{E}_{n}$.

Thus the $\left\{\Xi_{n l M}\right\}$ span the space $\underline{\mathcal{H}^{d+1, n}}$ and are a basis of this space, since their number is equal to its dimension.

We also need the following definitions:

Definition $6.2 \widetilde{\mathcal{E}}_{n}^{(1)}$ is the space of functions $f$ which are $\mathcal{C}^{\infty}$ and homogeneous of degree $1-n-d$ on $C_{+} \backslash\{0\}$ and satisfy

$$
\int_{\gamma} f(\xi)\left(\xi \cdot \xi^{\prime}\right)^{n} \alpha(\xi)=0 \quad \forall \xi^{\prime} \in C_{+} \backslash\{0\} .
$$

The integral in the lhs is independent of $\gamma$ if $f$ is homogeneous of degree $1-d-n$ so that the condition (6.12) is Lorentz invariant, and so is $\widetilde{\mathcal{E}}_{n}^{(1)}$. The integral in (6.12) is a homogeneous polynomial in $\xi^{\prime}$. if it vanishes for all $\xi^{\prime} \in C_{+} \backslash\{0\}$, by analytic continuation it also vanishes for all $\xi^{\prime} \in \mathbf{C}^{d+1}$ such that $\left(\xi^{\prime} \cdot \xi^{\prime}\right)=0$. According to (6.4),

$$
\mathcal{E}_{n}=\left\{f \in \mathcal{D}\left(X_{d}\right): \widetilde{f}_{1-d-n,+} \in \widetilde{\mathcal{E}}_{n}^{(1)}\right\} .
$$

Definition 6.3 $\widetilde{\mathcal{E}}_{n}^{(2)}$ is the space of functions $f$ which are $\mathcal{C}^{\infty}$ and homogeneous of degree $1-d-n$ on $C_{+} \backslash\{0\}$ and satisfy

$$
\int_{\gamma} f(\xi) p(\xi) \alpha(\xi)=0
$$

for any homogeneous polynomial $p$ of degree $n$ on $\mathbf{R}^{d+1}$.

It is equivalent to require this for all real or all complex homogeneous polynomial of degree $n$ on $\mathbf{R}^{d+1}$. Since $\xi \mapsto\left(\xi \cdot \xi^{\prime}\right)^{n}$ is such a polynomial, $\widetilde{\mathcal{E}}_{n}^{(2)} \subset \widetilde{\mathcal{E}}_{n}^{(1)}$. Again $\widetilde{\mathcal{E}}_{n}^{(2)}$ is Lorentz invariant. Taking in particular $\gamma=S_{0}$, we see that $\widetilde{\mathcal{E}}_{n}^{(2)}$ is the set of $\mathcal{C}^{\infty}$ homogeneous $f$ such that

$$
\int_{S_{0}} f(\xi) \boldsymbol{\xi}^{\alpha} d \boldsymbol{\xi}=0 \quad \forall \alpha \text { with }|\alpha| \leq n
$$

where $\alpha$ is a multi-index, $\boldsymbol{\xi}^{\alpha}=\prod_{j=1}^{d} \xi_{j}^{\alpha_{j}}$ and $|\alpha|=\sum_{j=1}^{d} \alpha_{j}$. Indeed $\boldsymbol{\xi}^{\alpha}$ is the restriction to $S_{0}$ of $\xi_{0}^{n-|\alpha|} \vec{\xi}^{\alpha}$, and conversely any monomial in $\xi$ of total degree $n$ is of this form. Any homogeneous polynomial $q$ of degree $\ell$ in $\vec{\xi} \in \mathbf{R}^{d}$ can be expressed as

$$
q(\vec{\xi})=\sum_{j=0}^{[\ell / 2]} r^{2 j} h_{\ell-2 j}(\vec{\xi}),
$$

where $r^{2}=\vec{\xi}^{2}$ and $h_{k}$ is a harmonic homogeneous polynomial of degree $k$ for $0 \leq k \leq \ell$ (see e.g. [41]). Since, on the unit sphere $S_{0}$, the restrictions of the harmonic homogeneous polynomial of degree $\ell$ are spanned by the hyperspherical harmonics $Y_{M}^{d, \ell}$ (see Appendix $\mathrm{A}$ ), the space $\widetilde{\mathcal{E}}_{n}^{(2)}$ is the set of $\mathcal{C}^{\infty}$ homogeneous $f$ of degree $1-d-n$ on $C_{+} \backslash\{0\}$ whose restrictions to $S_{0}$ have expansions into hyperspherical harmonics with vanishing components of order $\ell \leq n$. It is proved in Appendix C] that in fact $\widetilde{\mathcal{E}}_{n}^{(2)}=\widetilde{\mathcal{E}}_{n}^{(1)}$. 
Lemma 6.2 For all $f \in \widetilde{\mathcal{E}}_{n}^{(2)}$,

$$
\int_{\gamma_{1} \times \gamma_{2}} \overline{f(\xi)}(-1)^{n+1}\left(\xi \cdot \xi^{\prime}\right)^{n} \log \left(\xi \cdot \xi^{\prime}\right) f\left(\xi^{\prime}\right) \alpha(\xi) \alpha\left(\xi^{\prime}\right) \geq 0 .
$$

This expression vanishes if and only if $f=0$.

First, the lhs of this formula is independent of $\gamma_{1}$ and $\gamma_{2}$. For $j=1,2$, let $\gamma_{j}$ and $\gamma_{j}^{\prime}$ be smooth compact $(d-1)$-cycles in $C_{+} \backslash\{0\}$ such that $\gamma_{j}-\gamma_{j}^{\prime}=\partial B_{j}$ where $B_{j}$ is a bounded $d$-chain in $C_{+} \backslash\{0\}$. Denote $\varphi\left(\xi, \xi^{\prime}\right)=\overline{f(\xi)}(-1)^{n+1}\left(\xi \cdot \xi^{\prime}\right)^{n} \log \left(\xi \cdot \xi^{\prime}\right)$, and $\psi\left(\xi, \xi^{\prime}\right)=(-1)^{n+1}\left(\xi \cdot \xi^{\prime}\right)^{n} \log \left(\xi \cdot \xi^{\prime}\right) f\left(\xi^{\prime}\right)$. Then

$$
\begin{aligned}
& \left(\int_{\gamma_{1} \times \gamma_{2}}-\int_{\gamma_{1}^{\prime} \times \gamma_{2}^{\prime}}\right) \overline{f(\xi)}(-1)^{n+1}\left(\xi \cdot \xi^{\prime}\right)^{n} \log \left(\xi \cdot \xi^{\prime}\right) f\left(\xi^{\prime}\right) \alpha(\xi) \alpha\left(\xi^{\prime}\right)= \\
& \int_{B_{1} \times \gamma_{2}}\left(d_{\xi} \varphi\left(\xi, \xi^{\prime}\right) \alpha(\xi)\right) f\left(\xi^{\prime}\right) \alpha\left(\xi^{\prime}\right)+\int_{\gamma_{1}^{\prime} \times B_{2}} \overline{f(\xi)} \alpha(\xi)\left(d_{\xi^{\prime}} \psi\left(\xi, \xi^{\prime}\right) \alpha\left(\xi^{\prime}\right)\right) .
\end{aligned}
$$

Applying 4.10) we find

$$
d_{\xi} \varphi\left(\xi, \xi^{\prime}\right) \alpha(\xi)=\left(\xi^{0}\right)^{-1} \overline{f(\xi)}(-1)^{n+1}\left(\xi \cdot \xi^{\prime}\right)^{n} d \xi^{1} \wedge \ldots \wedge d \xi^{d}
$$

Since this is a homogeneous polynomial of degree $n$ in $\xi^{\prime}$, and $f \in \widetilde{\mathcal{E}}_{n}^{(2)}$, the second integral in (6.18) vanishes. The third integral similarly vanishes.

To prove the inequality [6.17] it suffices to do so in the case $\gamma_{1}=\gamma_{2}=S_{0}$, in which $\left(\xi \cdot \xi^{\prime}\right)=$ $1-\boldsymbol{\xi} \cdot \boldsymbol{\xi}^{\prime}$, and

$$
(-1)^{n+1}\left(\xi \cdot \xi^{\prime}\right)^{n} \log \left(\xi \cdot \xi^{\prime}\right)=\lim _{\rho \uparrow 1}(-1)^{n+1}\left(1-\rho \boldsymbol{\xi} \cdot \boldsymbol{\xi}^{\prime}\right)^{n} \log \left(1-\rho \boldsymbol{\xi} \cdot \boldsymbol{\xi}^{\prime}\right) .
$$

As the kernel of an operator on functions on $S_{0},\left(\boldsymbol{\xi} \cdot \boldsymbol{\xi}^{\prime}\right)$ is positive semi-definite since

$$
\int_{S_{0} \times S_{0}} \overline{h(\boldsymbol{\xi})}\left(\boldsymbol{\xi} \cdot \boldsymbol{\xi}^{\prime}\right) h\left(\boldsymbol{\xi}^{\prime}\right) d \boldsymbol{\xi} d \boldsymbol{\xi}^{\prime}=|V|^{2}, \quad V=\int_{S_{0}} h(\boldsymbol{\xi}) \boldsymbol{\xi} d \boldsymbol{\xi}
$$

Furthermore, $\left(\boldsymbol{\xi} \cdot \boldsymbol{\xi}^{\prime}\right)^{p}$ is also positive semi-definite for any integer $p \geq 0$ by a standard tensor product argument (a sketch of a proof may be found in Appendix C)

The inequality (6.17) finally follows from the following lemma

Lemma 6.3 For any integer $n \geq 0$ the power series expansion

$$
u_{n}(z)=(-1)^{n+1}(1-z)^{n} \log (1-z)=\sum_{m=0}^{\infty} u_{n, m} z^{m}
$$

converges for $|z|<1$ and satisfies $u_{n, m}>0$ for all $m>n$.

The convergence is obvious. Note that $u_{n}(0)=0$, i.e. $u_{n, 0}=0$, for $n \geq 0$. The statement holds for $n=0$. Suppose it holds for a certain $n \geq 0$. Then $u_{n+1}^{\prime}(z)=(n+1) u_{n}(z)-(z-1)^{n}$, so that $u_{(n+1), m}=(n+1) u_{n,(m-1)} / m>0$ for $m>n+1$. This proves the inequality (6.17). If this expression vanishes, then $f$ must satisfy, for all integers $N>n$

$$
\int_{S_{0} \times S_{0}} \overline{f\left(\boldsymbol{\xi}^{\prime}\right)}\left(\boldsymbol{\xi}^{\prime} \cdot \boldsymbol{\xi}\right)^{N} f(\boldsymbol{\xi}) d \boldsymbol{\xi} d \boldsymbol{\xi}^{\prime}=0
$$

Since $\left(\boldsymbol{\xi}^{\prime} \cdot \boldsymbol{\xi}\right)^{N}$ is a positive kernel this implies (by Schwartz's inequality)

$$
\int_{S_{0} \times S_{0}}\left(\boldsymbol{\xi}^{\prime} \cdot \boldsymbol{\xi}\right)^{N} f(\boldsymbol{\xi}) d \boldsymbol{\xi}=0 \quad \forall \boldsymbol{\xi}^{\prime} \in S_{0},
$$

so that, by Lemma C.1 of Appendix C $f$ is orthogonal to the homogeneous polynomials of degree $N$. Since $f \in \widetilde{\mathcal{E}}_{n}^{(2)}$ it is also orthogonal to the polynomials of degree $\leq n$, and it must vanish. This completes the proof of Lemma 6.2

We can now prove the main result of this Section: 
Lemma 6.4 The restriction of $\widehat{\mathcal{W}}_{n}$ to the Lorentz invariant subspace $\mathcal{E}_{n}$ of $\mathcal{D}\left(X_{d}\right)$ is positive semidefinite:

$$
\int_{X_{d} \times X_{d}} \overline{f(x)} \widehat{\mathcal{W}}_{n}\left(x, x^{\prime}\right) f\left(x^{\prime}\right) d x d x^{\prime} \geq 0, \quad f \in \mathcal{E}_{n} .
$$

This expression vanishes if and only if $\tilde{f}_{1-d-n,+}=0$.

Proof. By 4.16 4.17) and Lemma 4.1 for $z \in \mathcal{T}_{-}$and $z^{\prime} \in \mathcal{T}_{+}$,

$$
G_{\lambda}\left(z, z^{\prime}\right)=\frac{\Gamma(\lambda+d-1)^{2}}{2^{\lambda+2 d} \pi^{\frac{3 d-1}{2}} \Gamma\left(\lambda+\frac{d-1}{2}\right)} \int_{\gamma \times \gamma}(z \cdot \xi)^{1-d-\lambda}\left(\xi \cdot \xi^{\prime}\right)^{\lambda}\left(z^{\prime} \cdot \xi^{\prime}\right)^{1-d-\lambda} \alpha(\xi) \alpha\left(\xi^{\prime}\right) .
$$

According to the definition [5.5),

$$
\begin{aligned}
& \widehat{W}_{n}\left(z, z^{\prime}\right)=K_{n}^{\prime} G_{n}\left(z, z^{\prime}\right)+ \\
& +K_{n} \int_{\gamma \times \gamma}(-1)^{n+1}(-\log (z \cdot \xi))(z \cdot \xi)^{1-d-n}\left(\xi \cdot \xi^{\prime}\right)^{n}\left(z^{\prime} \cdot \xi^{\prime}\right)^{1-d-n} \alpha(\xi) \alpha\left(\xi^{\prime}\right) \\
& +K_{n} \int_{\gamma \times \gamma}(-1)^{n+1}(z \cdot \xi)^{1-d-n}\left(\xi \cdot \xi^{\prime}\right)^{n}\left(-\log \left(z^{\prime} \cdot \xi^{\prime}\right)\right)\left(z^{\prime} \cdot \xi^{\prime}\right)^{1-d-n} \alpha(\xi) \alpha\left(\xi^{\prime}\right) \\
& +K_{n} \int_{\gamma \times \gamma}(-1)^{n+1}(z \cdot \xi)^{1-d-n}\left(\xi \cdot \xi^{\prime}\right)^{n} \log \left(\xi \cdot \xi^{\prime}\right)\left(z^{\prime} \cdot \xi^{\prime}\right)^{1-d-n} \alpha(\xi) \alpha\left(\xi^{\prime}\right) .
\end{aligned}
$$

Here

$$
K_{n}^{\prime}=\left.\frac{(-1)^{n+1}}{n !} \frac{d}{d \lambda} \log \frac{\Gamma(\lambda+d-1)^{2}}{2^{\lambda+2 d} \pi^{\frac{3 d-1}{2}} \Gamma\left(\lambda+\frac{d-1}{2}\right)}\right|_{\lambda=n}, \quad K_{n}=\frac{\Gamma(n+d-1)^{2}}{2^{n+2 d} \pi^{\frac{3 d-1}{2}} n ! \Gamma\left(n+\frac{d-1}{2}\right)} .
$$

Boundary values can be taken on both sides of (6.27) as $z$ and $z^{\prime}$ tend to the reals. Thus, if $f, g \in \mathcal{S}\left(X_{d}\right)$,

$$
\begin{aligned}
& \left\langle\widehat{\mathcal{W}}_{n}, \bar{f} \otimes g\right\rangle=K_{n}^{\prime} \int_{X_{d} \times X_{d}} \overline{f(x)} G_{n}\left(x, x^{\prime}\right) g\left(x^{\prime}\right) d x d x^{\prime} \\
& +K_{n} \int_{X_{d} \times S_{0} \times S_{0}}(-1)^{n+1} \overline{f(x)}\left(-\log (x \cdot \xi)_{-}\right)(x \cdot \xi)_{-}^{1-d-n}\left(\xi \cdot \xi^{\prime}\right)^{n} \widetilde{g}_{+}\left(\xi^{\prime}, 1-d-n\right) d x d \boldsymbol{\xi} d \boldsymbol{\xi}^{\prime} \\
& +K_{n} \int_{S_{0} \times S_{0} \times X_{d}}(-1)^{n+1} \overline{\widetilde{f}_{+}(\xi, 1-d-n)}\left(\xi \cdot \xi^{\prime}\right)^{n}\left(-\log \left(x^{\prime} \cdot \xi\right)_{+}\right)\left(\xi \cdot x^{\prime}\right)_{+}^{1-d-n} g\left(x^{\prime}\right) d \boldsymbol{\xi} d \boldsymbol{\xi}^{\prime} d x^{\prime} \\
& +K_{n} \int_{S_{0} \times S_{0}}(-1)^{n+1} \overline{\widetilde{f}_{+}(\xi, 1-d-n)}\left(\xi \cdot \xi^{\prime}\right)^{n} \log \left(\xi \cdot \xi^{\prime}\right) \widetilde{g}_{+}\left(\xi^{\prime}, 1-d-n\right) d \boldsymbol{\xi} d \boldsymbol{\xi}^{\prime} .
\end{aligned}
$$

Suppose now that $f$ and $g$ belong to $\mathcal{E}_{n}$. Then $\widetilde{f}_{1-d-n, \pm}$ and $\widetilde{g}_{1-d-n, \pm}$ belong to $\widetilde{\mathcal{E}}_{n}^{(1)}=\widetilde{\mathcal{E}}_{n}^{(2)}$. The first term in (6.29) gives a contribution of 0 . The second term gives 0 because $\widetilde{g}_{+}\left(\xi^{\prime}, 1-d-n\right)$ is integrated against a homogeneous polynomial of degree $n$ in $\xi^{\prime}$. The third term in (6.29) similarly gives a contribution of 0 . Only the last term in (6.29) survives. By Lemma 6.2, if $g=f$, this term is non-negative and vanishes only if $\tilde{f}_{1-d-n,+}=0$. This completes the proof of Lemma 6.4

\subsection{Another proof of the positive-semi-definiteness of $\widehat{\mathcal{W}}_{n}$ on $\mathcal{E}_{n}$}

For every $f \in \mathcal{D}\left(X_{d}\right)$ we denote

$$
f_{l M}=\mathbf{V}_{l M} f, \quad f_{l M}(x)=\widetilde{f}_{l M}(t) Y_{l M}(\mathbf{x}) .
$$

The necessary and sufficient condition for $f$ to belong to $\mathcal{E}_{n}$ is that

$$
\int_{X_{d}} f_{l M}(x) \Xi_{l M}(x) d x=0 \text { i.e. } \int_{\mathbf{R}} \widetilde{f}_{l M}(t)(\operatorname{ch} t)^{d-1-\kappa} \mathbf{P}_{n+\kappa}^{-l-\kappa}(i \operatorname{sh} t) d t=0 \quad \forall l \leq n, \quad \forall M .
$$

Let also $\mathcal{W}_{\lambda l M}\left(x, x^{\prime}\right)=\mathbf{V}_{l M} \mathcal{W}_{\lambda}\left(x . x^{\prime}\right)=\left(\mathbf{V}_{l M} \mathcal{W}_{\lambda} \mathbf{V}_{l M}\right)\left(x . x^{\prime}\right)$ and similarly define $\widehat{\mathcal{W}}_{n l M}, G_{\lambda l M}$ We restrict $\lambda$ to vary in a small real open interval $\omega$ containing $n$. 
(1) Suppose $l>n$, hence also $l>\omega$. Let $f, g \in \mathcal{D}\left(X_{d}\right)$. For $\lambda \in \omega, \gamma_{l}(\lambda)>0$ and is analytic in $\lambda$, so that the pole removing procedure does nothing to $\mathcal{W}_{\lambda l M}\left(x, x^{\prime}\right)$ and

$$
\begin{aligned}
& \int_{X_{d} \times X_{d}} f(x) \widehat{\mathcal{W}}_{n l M}\left(x, x^{\prime}\right) \overline{g\left(x^{\prime}\right)} d x d x^{\prime}=\lim _{\lambda \rightarrow n} \int_{X_{d} \times X_{d}} f(x) \mathcal{W}_{\lambda l M}\left(x, x^{\prime}\right) \overline{g\left(x^{\prime}\right)} d x d x^{\prime} \\
& =\int_{X_{d} \times X_{d}} \gamma_{l}(n) f_{l M}(x) \Xi_{n l M}(x) \overline{\Xi_{n l M}\left(x^{\prime}\right) g_{l M}\left(x^{\prime}\right)} d x d x^{\prime},
\end{aligned}
$$

and this is non-negative if $g=f$.

(2) If $l \leq n$ and $f, g \in \mathcal{E}_{n}$,

$$
\begin{aligned}
& \int_{X_{d} \times X_{d}} f(x) \widehat{\mathcal{W}}_{n l M}\left(x, x^{\prime}\right) g\left(x^{\prime}\right) d x d x^{\prime}= \\
& \left.\frac{(-1)^{n+1}}{n !} \int_{X_{d} \times X_{d}} f_{l M}(x) \partial_{\lambda}\left[A_{1}(\lambda, l) \Xi_{\lambda l M}(x) \Xi_{\lambda l M}\left(x^{\prime}\right)\right]\right|_{\lambda=n} g_{l M}\left(x^{\prime}\right) d x d x^{\prime}
\end{aligned}
$$

The derivative of the bracket at $\lambda=n$ is a sum of terms each of which contains at least one undifferentiated factor $\Xi_{n l M}(x)$ or $\Xi_{n l M}\left(x^{\prime}\right)$, and the whole expression is therefore equal to 0 .

\section{Hilbert space structure}

The status of the de Sitter tachyons described up to here exhibits a striking analogy with gauge QFT 19, 20, in that it is impossible to reconcile positive-semi-definiteness (which would provide a direct probabilistic interpretation of the states), Lorentz invariance and local commutativity. Furthermore, as in QED, to preserve Lorentz invariance and local commutativity of the fields it has been necessary to introduce an anomalous term in the field equations.

There is now an additional complication that has to be faced: the choice of working in a local and covariant gauge supplies only the linear set of local states, i.e. those states that can be obtained by applying polynomials of the field operators to the vacuum. This set is endowed with a non-positive inner product which encodes the knowledge of the Wightman functions. In general, to identify the full set of physical states of the theory it is necessary to consider limits and take in some sense the closure of the local states; another piece of information is needed which is not contained in the field equations: a (Hilbert space) topology compatible with the Wightman functions has to be assigned on the local states. For example the construction of the charged stated in QED - which should be possible at the price of breaking Lorentz invariance - and in QCD - which should be impossible, providing this way the yet lacking proof of quark confinement - requires taking limits, such as the so called "particle behind the moon" procedure: those fundamental questions cannot be decided without completing the local states in a suitable Hilbert topology.

A general framework to deal with this kind of problems has been introduced in [54, 55. In this Section we will use that scheme to construct Krein spaces for the free fields associated to the two-point functions $\widehat{\mathcal{W}}_{n}$; the case $n=0, d=2$ has been already sketched in [56].

\subsection{Preliminaries}

In this subsection $l$ is always $\leq n$. The first preliminary observation is that $(\operatorname{ch} t)^{-l} h_{n l}(t)$ is a real polynomial in sht. Indeed, we saw in Eq. (6.6) that the function $\Xi_{n l M}(x)$ extends to a real pseudo-harmonic polynomial on $M_{d+1}$ and its restriction to $X_{d}$ satisfies the Klein-Gordon equation. Since

$$
\Xi_{n l M}(x)=h_{n, l}(t) Y_{l M}(\mathbf{x})=h_{n l}(t)(\operatorname{ch} t)^{-l} Y_{l M}(\vec{x}),
$$

and $Y_{l M}(\vec{x})$ is a polynomial in $\vec{x}$, we infer that $(\operatorname{ch} t)^{-l} h_{n l}(t)$ must be a polynomial in $x^{0}=\operatorname{sh} t$. By taking as before $z=-i \operatorname{sh} t$ and assuming that $\operatorname{Re} t>0, \operatorname{Im} t>0$ small enough, i.e. $\operatorname{Im} z<0$, $\operatorname{Re} z>0$ small enough, we have

$$
\begin{aligned}
& (\operatorname{ch} t)^{-l} h_{n l}(t)=e^{\frac{i \pi}{2}(n-l)}\left(1-z^{2}\right)^{\frac{1}{2}\left(-l-\frac{d-2}{2}\right)} \mathbf{P}_{n+\frac{d-2}{2}}^{-l-\frac{d-2}{2}}(z) \\
& =\frac{2^{l+\frac{d-2}{2}} \Gamma\left(l+\frac{d-1}{2}\right) \Gamma(n-l+1)}{\pi^{1 / 2} \Gamma(n+l+d-1)} i^{n-l} C_{n-l}^{l+\frac{d-1}{2}}(-i \operatorname{sh} t)
\end{aligned}
$$


Since $r^{N} C_{N}^{\nu}(z / r)$ is a polynomial in $z$ and $r^{2}$, real if $\nu$ is real, it follows that $(\operatorname{ch} t)^{-l} h_{n l}(t)$ is a real polynomial of degree $(n-l)$ in sh $t$. The above formulae show that $\Xi_{n l M}(x)$ coincides with the polynomial $Y_{L}^{d+1, n}\left(x^{0}, \vec{x}\right)$ defined in (A.16) up to a constant factor.

The next observation is that there exists a collection of real functions $g_{l} \in \mathcal{S}(\mathbf{R})$, with $0 \leq l \leq n$, such that, denoting

$$
\chi_{l M}(x)=g_{l}(t) Y_{l M}(\mathbf{x}),
$$

the following conditions are satisfied :

$$
\int_{X_{d}} \Xi_{n l M}(x) \chi_{l^{\prime} M^{\prime}}(x) d x=\delta_{l l^{\prime}} \delta_{M M^{\prime}} \quad \text { for all } l, l^{\prime}=0, \ldots, n, \quad \text { and all } M, M^{\prime}
$$

and

$$
\left\langle\chi_{l M}, \chi_{l^{\prime} M^{\prime}}\right\rangle=\int_{X_{d} \times X_{d}} \overline{\chi_{l M}(x)} \widehat{\mathcal{W}}_{n}\left(x, x^{\prime}\right) \chi_{l^{\prime}, M^{\prime}}\left(x^{\prime}\right) d x d x^{\prime}=0 \quad \forall M, M^{\prime} .
$$

There is a large arbitrariness in the choice of the functions $g_{l}$. They will be chosen to belong to $\mathcal{D}(\mathbf{R})$. The problem of their existence can be reformulated as follows: let

$$
H_{\lambda l}=\int_{\mathbf{R}} g_{l}(t) h_{\lambda l}(t)(\operatorname{ch} t)^{d-1} d t ;
$$

Eqs. (4.27) and (5.1), the definition (5.5) of $\widehat{\mathcal{W}}_{n}$ and the orthonormality of the $\left\{Y_{l M}\right\}$, imply that the above requirements will be satisfied if

$$
H_{n l}=1
$$

and

i.e.

$$
\left.2 \operatorname{Re} \frac{\partial}{\partial \lambda} H_{\lambda, l}\right|_{\lambda=n}=-\left.\frac{\frac{\partial}{\partial \lambda} A_{1}(\lambda, l)}{A_{1}(\lambda, l)}\right|_{\lambda=n}
$$

$$
\left.\int_{\mathbf{R}} g_{l}(t) \operatorname{Re} \partial_{\lambda} h_{\lambda, l}(t)(\operatorname{ch} t)^{d-1} d t\right|_{\lambda=n}=-\left.\frac{\frac{\partial}{\partial \lambda} A_{1}(\lambda, l)}{2 A_{1}(\lambda, l)}\right|_{\lambda=n},
$$

We take $\lambda$ real and close to $n$ in these conditions. If two real distributions $t, t^{\prime} \in \mathcal{S}^{\prime}(\mathbf{R})$ are linearly independent, then for arbitrary real numbers $a_{1}, a_{2}$ there exists a real test function $\varphi \in \mathcal{S}(\mathbf{R})$ such that $(t, \varphi)=a_{1}$ and $\left(t^{\prime}, \varphi\right)=a_{2}$. Thus the existence of $g_{l}$ is guaranteed if we show that $t \mapsto h_{n l}(t)$ and $\left.t \mapsto \operatorname{Re} \partial_{\lambda} h_{\lambda l}(t)\right|_{\lambda=n}$ are linearly independent. This is done in Appendix D.

By expanding the rhs of Eq. (5.10) using (5.1) we may rewrite it as follows

$$
\begin{aligned}
\left(\square_{x, x^{\prime}}-n(n+d-1)\right) \widehat{\mathcal{W}}_{n}\left(x, x^{\prime}\right) & =\sum_{l=0}^{n} A(l) \sum_{M} \Xi_{n l M}(x) \Xi_{n l M}\left(x^{\prime}\right), \\
A(l)=\frac{(-1)^{n+1}}{n !}(2 n+d-1) A_{1}(n, l) & =\frac{(-1)^{n-l+1}(2 n+d-1) \Gamma(l+n+d-1) \Gamma(n+1)}{2 n ! \Gamma(n+1-l)} .
\end{aligned}
$$

\subsection{Krein spaces - infrared states}

In the following we abbreviate $\Xi_{n l M}$ as $\Xi_{l M}$. $\mathcal{J}$ is the set of all pairs $l, M$ where $0 \leq l \leq n$ and $M$ is a multi-index associated to $l$; where no confusion arises an element of $\mathcal{J}$ will be denoted $j, k, r$, etc. If $j=\{l, M\}$ and $k=\{r, L\}$ are elements of $\mathcal{J}$, we denote $\delta_{j k}=\delta_{l r} \delta_{M L}$ and $A(j)=A(l)$. We define an infrared regular part $f_{0}$ of $f$ by

$$
f_{0}(x)=f(x)-\sum_{j \in \mathcal{J}} c_{j}(f) \chi_{j}(x)
$$

where

$$
c_{j}(f)=\int_{X_{d}} \Xi_{j}(x) f(x) d x, \quad(j \in \mathcal{J})
$$


$f_{0}$ belongs to $\mathcal{E}_{n}$, since it is orthogonal to all the $\Xi_{j}$. The map $f \rightarrow f_{0}$ is a projection onto $\mathcal{E}_{n}$ parallel to the subspace spanned by the $\chi_{j}$, in particular $\chi_{j 0}=0$. It follows from the above equations that

$$
\left\langle\left(\square+m_{n}^{2}\right) f, g\right\rangle=\int_{X_{d} \times X_{d}} \sum_{j \in \mathcal{J}} A(j) \overline{f(x)} \Xi_{j}(x) \Xi_{j}\left(x^{\prime}\right) g\left(x^{\prime}\right) d x d x^{\prime}=\sum_{j \in \mathcal{J}} A(j) \overline{c_{j}(f)} c_{j}(g) .
$$

We are now ready for the construction of the Hilbert-Krein topology. In the first step we use the above projection to single out the infrared regular part in the inner product 6.2) associated to the Wightman function:

$$
\langle f, g\rangle=\left\langle f_{0}, g_{0}\right\rangle+\sum_{j \in \mathcal{J}} \overline{c_{j}(f)}\left\langle\chi_{j}, g\right\rangle+\sum_{j \in \mathcal{J}} c_{j}(g)\left\langle f, \chi_{j}\right\rangle .
$$

In the second step we introduce a pre-Hilbert product on the full space of test functions $\mathcal{D}\left(X_{d}\right)$ as follows:

$$
(f, g)=\left\langle f_{0}, g_{0}\right\rangle+\sum_{j \in \mathcal{J}} \overline{c_{j}(f)} c_{j}(g)+\sum_{j \in \mathcal{J}}\left\langle f, \chi_{j}\right\rangle\left\langle\chi_{j}, g\right\rangle
$$

The majorization

$$
|\langle f, g\rangle|^{2} \leq(f, f)(g, g)
$$

holds on test functions and shows the compatibility of the Hilbert topology with the Wightman functions. The ideal of the pre-Hilbert product is the subspace

$$
\mathcal{I}=\left\{f \in \mathcal{E}_{n},\langle f, f\rangle=0,\left\langle\chi_{j}, f\right\rangle=0 \quad \forall j \in \mathcal{J}\right\} .
$$

By standard Hilbert space techniques, taking the quotient $\mathcal{D}\left(X_{d}\right) / \mathcal{I}$ and completing it we get a Hilbert space which we denote $\mathcal{K}^{1}$. The Wightman inner product can be uniquely continuously extended to the Hilbert space $\mathcal{K}^{1}$. The linear functionals $f \mapsto\left\langle\chi_{j}, f\right\rangle$ are continuous in the topology of $\mathcal{K}^{1}$ by (7.17) hence there is, for each $j \in \mathcal{J}$, a unique vector $v_{j} \in \mathcal{K}^{1}$ such that

$$
\left\langle\chi_{j}, f\right\rangle=\left(v_{j}, f\right) .
$$

The vectors $v_{j}$ are referred to as infrared states. In the closely similar case of the massless twodimensional Klein-Gordon field on the Minkowski space-time there exists only one such vector; it corresponds to an infinitely delocalized state in the sense that it cannot be obtained by applying a localized field to the vacuum but only as an infrared limit [55]. On the contrary, here the vectors $v_{j}$ can be identified with specific test functions:

$$
v_{j}=\frac{1}{A(j)}\left(\square+m_{n}^{2}\right) \chi_{j}
$$

Let indeed $v_{j}^{\prime}$ denote the rhs of (7.20). Since $\Xi_{k}$ is a solution of the Klein-Gordon equation, $c_{k}\left(v_{j}^{\prime}\right)=0$ for every $k \in \mathcal{J}$, hence $v_{j 0}^{\prime}=v_{j}^{\prime}$ and

$$
\left(v_{j}^{\prime}, f\right)=\left\langle A(j)^{-1}\left(\square+m_{n}^{2}\right) \chi_{j}, f_{0}\right\rangle+\sum_{k \in \mathcal{J}}\left\langle v_{j}^{\prime}, \chi_{k}\right\rangle\left\langle\chi_{k}, f\right\rangle
$$

The first term in the rhs is zero by applying (7.14) since $c_{k}\left(f_{0}\right)=0$. For the other terms,

$$
\left\langle v_{j}^{\prime}, \chi_{k}\right\rangle=\left\langle A(j)^{-1}\left(\square+m_{n}^{2}\right) \chi_{j}, \chi_{k}\right\rangle=A(j)^{-1} \sum_{r \in \mathcal{J}} A(r) \overline{c_{r}\left(\chi_{j}\right)} c_{r}\left(\chi_{k}\right)=\delta_{j k}
$$

since $c_{r}\left(\chi_{j}\right)=\delta_{r j}$. Therefore $\left(v_{j}^{\prime}, f\right)=\left\langle\chi_{j}, f\right\rangle$, i.e. we can take $v_{j}=v_{j}^{\prime}$. Note that $v_{j} \in \mathcal{E}_{n}$ and

$$
\left(v_{j}, \chi_{k}\right)=0, \quad\left\langle v_{j}, \chi_{k}\right\rangle=\left(v_{j}, v_{k}\right)=\delta_{j k}, \quad\left\langle v_{j}, v_{k}\right\rangle=0, \quad\left(\chi_{j}, \chi_{k}\right)=\delta_{j k},
$$

and

$$
\left\langle v_{j}, f\right\rangle=c_{j}(f), \quad\left(\chi_{j}, f\right)=\left\langle v_{j}, f\right\rangle .
$$

The result of this Section may be summarized in the following 
Proposition 7.1 The Hilbert space $\mathcal{K}^{1}$ can be written as a direct sum:

$$
\mathcal{K}^{1}={\overline{\mathcal{E}_{n}^{\perp}}}^{\langle\cdot, \cdot\rangle} \oplus V_{n} \oplus X_{n}
$$

where $\mathcal{E}_{n}^{\perp}$ is the subspace of $\mathcal{E}_{n}$ orthogonal to $V_{n}, V_{n}$ is the linear span of the set $\left\{v_{j}\right\}$ and $X_{n}$ is the linear span of the set $\left\{\chi_{j}\right\}$.

The metric operator $\eta^{(1)}$ representing $\langle\cdot, \cdot\rangle$ in the Hilbert product $(\cdot, \cdot)$ is given by

$$
\eta^{(1)}=\left[\begin{array}{lll}
\mathbb{I} & 0 & 0 \\
0 & 0 & \mathbb{I} \\
0 & \mathbb{I} & 0
\end{array}\right]
$$

$\eta^{(1)} \eta^{(1)}=\mathbb{I}$ so that $\mathcal{K}^{1}$ is a Krein space.

\section{3 de Sitter Symmetry}

The functions $\Xi_{j}$, considered as polynomials on $M_{d+1}$, form a basis of the space of homogeneous pseudo-harmonic polynomials of degree $n$ on $M_{d+1}$ (see Appendix $\mathbf{A}$ ); on this space the quasi-regular representation of the Lorentz group reduces to an irreducible representation. Hence there exists a matricial representation $L_{+}^{\uparrow} \ni \Lambda \mapsto S(\Lambda)$ such that

$$
\Xi_{j}\left(\Lambda^{-1} x\right)=\sum_{k \in \mathcal{J}} S_{j k}(\Lambda) \Xi_{k}(x)
$$

for all $j \in \mathcal{J}$. The matrix elements $S_{j k}(\Lambda)$ are real polynomials in the matrix elements of $\Lambda$. Let

$$
\delta \chi_{j}(x, \Lambda)=\chi_{j}(\Lambda x)-\sum_{k \in \mathcal{J}} S_{j k}^{T}(\Lambda) \chi_{k}(x)
$$

The function $\delta \chi_{j}(\cdot, \Lambda)$ belongs to $\mathcal{E}_{n}$ :

$c_{k}\left(\delta \chi_{j}(\cdot, \Lambda)\right)=\int_{X_{d}} \delta \chi_{j}(x, \Lambda) \Xi_{k}(x) d x=\int_{X_{d}} \chi_{j}(x) \Xi_{k}\left(\Lambda^{-1} x\right) d x-\sum_{r \in \mathcal{J}} S_{r j}(\Lambda) \int_{X_{d}} \chi_{r}(x) \Xi_{k}(x) d x=0$.

Let now $f$ be an element of the ideal $\mathcal{I}$ (see (7.18) $)$ and let $f_{\Lambda}(x)=f\left(\Lambda^{-1} x\right)$. Obviously $f_{\Lambda} \in \mathcal{E}_{n}$ and $\left\langle f_{\Lambda}, f_{\Lambda}\right\rangle=0$. Since the product $\langle\cdot, \cdot\rangle$ is positive semi-definite on $\mathcal{E}_{n},\langle f, g\rangle=0$ for all $g \in \mathcal{E}_{n}$, hence

$$
\left\langle f, \delta \chi_{j}(\cdot, \Lambda)\right\rangle=\left\langle f_{\Lambda}, \chi_{j}\right\rangle=0
$$

and therefore also $f_{\Lambda} \in \mathcal{I}$. The regular representation of the Lorentz group naturally defined on $\mathcal{D}\left(X_{d}\right)$ (see 2.15) is thus compatible with the procedure of quotienting and completing which leads to $\mathcal{K}^{1}$, and this provides a natural continuous representation $U$ of the de Sitter group on $\mathcal{K}^{1}$ that preserves the sesquilinear form $\langle\cdot, \cdot\rangle$. However $U$ is not unitary. The following proposition characterizes the action of the Lorentz group on the infrared states:

Property 7.1 The infrared states span a Lorentz invariant subspace of the Krein one particle space $\mathcal{K}^{1}$.

Define indeed

$$
\delta v_{j}(x, \Lambda)=\frac{1}{A(j)}\left(\square+m_{n}^{2}\right) \delta \chi_{j}(x, \Lambda)=v_{j}(\Lambda x)-\sum_{k \in \mathcal{J}} S_{j k}^{T}(\Lambda) v_{k}(x) .
$$

$\delta v_{j}(\cdot, \Lambda)$ manifestly belongs to $\mathcal{E}_{n}$. Furthermore, for any $f \in \mathcal{D}\left(X_{d}\right)$,

$$
\left\langle\delta v_{j}, f\right\rangle=\frac{1}{A(j)}\left\langle\left(\square+m_{n}^{2}\right) \delta \chi_{j}, f\right\rangle=0
$$

by applying (7.14) and (7.29). It follows that $\left(\delta v_{j}, f\right)=0$ for every $f \in \mathcal{D}\left(X_{d}\right)$. This means that

$$
U(\Lambda) v_{j}=\sum_{k} S_{k j}^{-1}(\Lambda) v_{k}=\left(S^{-1 T}(\Lambda) v\right)_{j}
$$




\subsection{The role of the infrared operators: field equation and Gupta- Bleuler condition}

The Krein-Fock space $\mathcal{K}$ is obtained from the "one-particle" space $\mathcal{K}^{1}$ by the standard construction [18. The one-particle scalar product $\langle f, g\rangle$ extends to a continuous non degenerate sesquilinear form on $\mathcal{K}$, also denoted $\langle\cdot, \cdot\rangle$ and called the Wightman product. The scalar tachyon field $\phi(x)$ is represented in $\mathcal{K}$ by formulae of the usual kind. In particular $\phi$ may be split into its creation and annihilation parts $\phi(f)=\phi^{+}(f)+\phi^{-}(f)$, where

$$
\begin{aligned}
& \left.\left(\phi(f)^{+} \Psi_{h}\right)^{(n)}\left(x_{1}, \ldots, x_{n}\right)=\frac{1}{\sqrt{n}} \sum_{j=1}^{n} f\left(x_{j}\right) h^{(n-1)}\left(x_{1}, \ldots, \hat{x}_{j}, \ldots, x_{n}\right),\right) \\
& \left(\phi(f)^{-} \Psi_{h}\right)^{(n)}\left(x_{1}, \ldots, x_{n}\right)=\sqrt{n+1} \int f(x) \widehat{\mathcal{W}}\left(x, x^{\prime}\right) h^{(n+1)}\left(x^{\prime}, x_{1}, \ldots, x_{n}\right) .
\end{aligned}
$$

It follows, as usual, that

$$
\left[\phi^{+}(x), \phi^{+}\left(x^{\prime}\right)\right]=\left[\phi^{-}(x), \phi^{-}\left(x^{\prime}\right)\right]=0, \quad\left[\phi^{-}(x), \phi^{+}\left(x^{\prime}\right)\right]=\widehat{\mathcal{W}}_{n}\left(x, x^{\prime}\right)
$$

so that

$$
\left[\phi(x), \phi\left(x^{\prime}\right)\right]=\widehat{\mathcal{W}}_{n}\left(x, x^{\prime}\right)-\widehat{\mathcal{W}}_{n}\left(x^{\prime}, x\right)=C_{n}\left(x, x^{\prime}\right)
$$

The infrared operators $\phi\left(v_{j}\right)$ belong to the center of the field algebra, i.e. they commute with the operator $\phi(f)$ for every $f \in \mathcal{D}\left(X_{d}\right)$ :

$$
\left[\phi\left(v_{j}\right), \phi(f)\right]=\frac{1}{A(j)} \int_{X_{d} \times X_{d}}\left[\left(\square+m_{n}^{2}\right) \chi_{j}(x)\right] C_{n}\left(x, x^{\prime}\right) f\left(x^{\prime}\right) d x^{\prime}=0
$$

since $\left(\square+m_{n}^{2}\right) C_{n}\left(x, x^{\prime}\right)=0$.

Using (7.24), Eq. (7.14) can be rewritten as follows:

$$
\int_{X_{d}} \overline{f(x)}\left\langle\left(\square+m_{n}^{2}\right) \phi(x) \Omega, \phi(g) \Omega\right\rangle d x=\int_{X_{d}} \overline{f(x)} \sum_{j \in \mathcal{J}} A(j) \Xi_{j}(x)\left\langle\phi\left(v_{j}\right) \Omega, \phi(g) \Omega\right\rangle d x .
$$

The non-degeneracy of the Wightman product and the factorization property of the $n$-point functions then allow us to conclude that the tachyon field satisfies the non-homogeneous equation

$$
\left(\square+m_{n}^{2}\right) \phi(x)=\sum_{j \in \mathcal{J}} A(j) \phi\left(v_{j}\right) \Xi_{j}(x) \equiv \Theta_{n}(x) .
$$

This allows us to reinterpret the whole construction as a Gupta-Bleuler quantization of the tachyons: the physical states of the theory are annihilated by the destruction part of the rhs of Eq. (7.38):

$$
\Theta(x)^{-}|p h y s\rangle=0
$$

Indeed the physical states in $\mathcal{K}_{1}$ are the closure of $\mathcal{E}_{n}$ in the Hilbert space topology and, by (7.24), they are exactly the vectors in $\mathcal{K}_{1}$ which are annihilated by all the operators $\phi\left(v_{j}\right)^{-}, j \in \mathcal{J}$. Hence due to the Fock space construction (see (7.34), (7.35)), the physical states are those which are annihilated by all the operators $\phi\left(v_{j}\right)^{-}, j \in \mathcal{J}$. Thus they are also annihilated by $\Theta(x)^{-}$for all $x$, and the converse is also true by smearing $\Theta(x)^{-}$with $\chi_{j}(x)$. As a consequence, the usual homogeneous field equation holds on physical states, similarly to what happens with the Gauss law in QED [19, 20;

$$
\left\langle p h y s\left|\left(\square+m_{n}^{2}\right) \phi(x)\right| p h y s\right\rangle=0
$$

\subsection{Gauge transformations}

There is indeed a gauge symmetry preserving the field equation (7.40):

$$
\phi(x) \longrightarrow \phi(x)+\sum \lambda_{j} \Xi_{j}(x) .
$$

The infrared operators may be used to define the gauge charge operators

$$
Q_{j}=\frac{1}{2 i}\left[\phi^{+}\left(v_{j}\right)-\phi^{-}\left(v_{j}\right)\right]
$$


that act as generators of the above gauge transformations. Indeed, by Eq. (7.36),

$$
\left[\phi^{ \pm}\left(v_{j}\right), \phi(f)\right]=\mp\left\langle v_{j} f\right\rangle=\mp c_{j}(f)=\mp \int_{X_{d}} \Xi_{j}(x) f(x) d x
$$

and therefore

$$
\left[Q_{j}, \phi(f)\right]=i c_{j}(f), \quad\left[Q_{j}, \phi(x)\right]=i \Xi_{j}(x) .
$$

Is there any conserved current associated to the above charges? Suppose that $f$ is a smooth solution of the Klein-Gordon equation and $\Sigma$ a smooth space-like hypersurface homotopic to the sphere $\left\{x: x^{0}=0\right\}$ in $X_{d}$. Then

$$
\int_{\Sigma} f(x) \overleftrightarrow{\partial_{\mu}} C_{n}(x, y) d \Sigma^{\mu}(x)=i f(y)
$$

Indeed, since $f$ and $C_{n}(x, y)$ are solutions of the Klein-Gordon equation, the lhs is independent of $\Sigma$, and we can choose $\Sigma=\left\{x \in X_{d}: x^{0}=y^{0}\right\}$. Then the result follows from the equal-time commutation relations. (3.20). This holds for any mass. For each $k \in \mathcal{J}$ let

$$
j_{k \mu}(x)=\Xi_{k}(x) \overleftrightarrow{\partial_{\mu}} \phi(x)
$$

Then, with $\Sigma$ as above we find

$$
\left[\int_{\Sigma} j_{k \mu}(x) d \Sigma^{\mu}(x), \phi(y)\right]=i \Xi_{k}(y) .
$$

The integral in this formula has the same commutator with $\phi$ as $Q_{k}$. However $j_{k \mu}(x)$ is not a conserved current since $\phi$ is not a solution of the Klein-Gordon equation. By (7.40)

$$
\nabla^{\mu} j_{k \mu}(x)=-\Xi_{k}(x) \sum_{j \in \mathcal{J}} A(j) \phi\left(v_{j}\right) \Xi_{j}(x)
$$

The function $x \mapsto \Xi_{k}(x) \Xi_{j}(x)$ is the restriction to $X_{d}$ of a homogeneous polynomial of degree $2 n$ on $M_{d+1}$. We can apply the following lemma:

Lemma 7.1 Let $q$ be a polynomial of degree $m \geq 0$ on $M_{d+1}$. There exists a $\mathcal{C}^{\infty}$ function $f$ on $X_{d}$ such that $\square_{d S} f=q \mid X_{d}$.

Proof If $h$ is a homogeneous pseudo-harmonic polynomial of degree $s>0$, i.e. $\square_{\text {Mink }} h=0$, then, as mentioned in Subsect. 2.2 $\left(h \mid X_{d}\right)=\square_{d S}[s(s+d-1)]^{-1}\left(h \mid X_{d}\right)$. If $h$ is a constant, we may use (5.10) with $n=0$, i.e. if $z^{\prime} \in \mathcal{T}_{+}$,

$$
\square_{x} \widehat{W}_{0}\left(x, z^{\prime}\right)=-\frac{\Gamma(d)}{4 \pi^{\frac{d}{2}}},
$$

so the statement also holds in this case. For a general homogeneous polynomial $q$ of degree $m \geq 0$ on $M_{d+1}$, there exists (see [41, p. 441]) a unique expansion

$$
q(x)=\sum_{k=0}^{[m / 2]}(x \cdot x)^{k} h_{m-2 k}(x),
$$

where each $h_{m-2 k}$ is a pseudo-harmonic homogeneous polynomial of degree $m-2 k$, so that

$$
q\left|X_{d}=\sum_{k=0}^{[m / 2]}(-1)^{k} h_{m-2 k}\right| X_{d} .
$$

Therefore the statement of the lemma holds.

By virtue of this lemma, there exist $\mathcal{C}^{\infty}$ functions $\varphi_{k j}$ on $X_{d}$ such that $\Xi_{k} \Xi_{j}=\square_{d S} \varphi_{k j}$. We can now define

$$
j_{k \mu}^{\prime}(x)=j_{k \mu}(x)+\sum_{j \in \mathcal{J}} A(j) \phi\left(v_{j}\right) \partial_{\mu} \varphi_{k j}(x) .
$$

The current $j_{k \mu}^{\prime}(x)$ has the same commutation properties as $j_{k \mu}(x)$, but $\nabla^{\mu} j_{k \mu}^{\prime}(x)=0$. 


\subsection{The case $n=1$}

The scalar tachyon whose squared mass is equal to the opposite of the space-time dimension $m_{1}^{2}=-d$ plays a role in the construction of the de Sitter linearized quantum gravity [8, 9]. In this context the $d+1$ implementable gauge transformations are actually interpretable as describing a residual conformal symmetry. Let us summarize here the nice geometrical construction underlying this theory. The pseudo-harmonic homogeneous polynomials of degree $n=1$ on $M_{d+1}$ are just the linear homogeneous polynomials and their space is spanned by $x_{0}, \ldots, x_{d}$. It is easy to check that $\Xi_{00}=$ Const. $x_{0}$, while the other $\Xi_{l M}$ are proportional to the other coordinate functions $x_{j}$, $1 \leq j \leq d$. The set of indices $\mathcal{J}$ becomes therefore identical to the set $\{0,1, \ldots, d\}$. Its elements will be denoted with latin letters to avoid confusions with (arbitrary) coordinates on $X_{d}$. It is in fact convenient in this case to renormalize the $\Xi_{j}(x)$ so that $\Xi_{j}(x)=x_{j}(0 \leq j \leq d)$, and contragrediently renormalize the $\chi^{j}$ and $v^{j}$. We now have the following forms of (5.3) and (17.10) (see (5.10)

$$
\begin{gathered}
G_{1}\left(x, x^{\prime}\right)=\frac{\Gamma(d-1)}{(4 \pi)^{d / 2}} C_{1}^{\frac{d-1}{2}}\left(x \cdot x^{\prime}\right)=\frac{\Gamma(d)}{(4 \pi)^{d / 2}}\left(x \cdot x^{\prime}\right), \\
\left(\square_{x, x^{\prime}}-d\right) \widehat{\mathcal{W}}_{n}\left(x, x^{\prime}\right)=\frac{(d+1) \Gamma(d)}{(4 \pi)^{d / 2}}\left(x \cdot x^{\prime}\right)=\sum_{j} \frac{(d+1) \Gamma(d)}{(4 \pi)^{d / 2}} x_{j} \eta^{j j} x_{j}^{\prime},
\end{gathered}
$$

i.e. $A(l)$ has become $(-1)^{l}(4 \pi)^{-d / 2}(d+1) \Gamma(d)$, and $A(j)=\eta^{j j}(4 \pi)^{-d / 2}(d+1) \Gamma(d)$. In particular

$$
v^{j}=\eta^{j j} \frac{(4 \pi)^{d / 2}}{(d+1) \Gamma(d)}(\square-d) \chi^{j} .
$$

In the case $n=1$ the gauge invariance is related to the existence on $X_{d}$ of $(d+1)$ linearly independent "conformal-Killing vector fields" (not included in the list of the true Killing vector fields, which generate the Lorentz transformations). Their role has been emphasized by [8] and [9]. A vector field $w$ on $X_{d}$ is a conformal-Killing vector field if it satisfies

$$
\nabla_{\mu} w_{\nu}+\nabla_{\nu} w_{\mu}=g_{\mu \nu} f, \quad f=\frac{2}{d} \nabla^{\mu} w_{\mu}
$$

where the last equality follows from the first. If we impose on $w$ the further requirement that

$$
w_{\mu}=\partial_{\mu} h,
$$

where $h$ is some $\mathcal{C}^{\infty}$ function on $X_{d}$, this function must satisfy

$$
\nabla_{\mu} \nabla_{\nu} h=\frac{g_{\mu \nu}}{d} \square h
$$

Conversely if a function $h$ satifies the above equation, then $w_{\mu}=\partial_{\mu} h$ satisfies (7.58) with $f=\frac{2}{d} \square h$. If, furthermore, $\square h=d h$, then $f=2 h$. It will now be shown that (7.60) is satisfied by $h=x_{j}=\Xi_{j}$, i.e. any of the Minkowskian coordinates. By symmetry and analyticity it is sufficient to show this for $x_{0}$. We already know that $(\square-d) x_{j}=0$. We thus need to prove that

$$
\nabla_{\mu} \nabla_{\nu} x_{0}=g_{\mu \nu} x_{0}
$$

By analytic continuation, it suffices to prove this in one analytic coordinate patch. We choose (as independent coordinates on $\left.X_{d}\right) x^{0}, \ldots, x^{d-1}$, with $\left(x^{d}\right)^{2}=1+\sum_{\mu=0}^{d-1} \eta_{\mu \mu}\left(x^{\mu}\right)^{2}$ in the region $x^{d}>0$. Greek indices will take the values $0, \ldots, d-1$. We continue to denote $x_{\mu}=\eta_{\mu \mu} x^{\mu}$ (no sum). We then find

$$
\begin{aligned}
& g_{\mu \nu}(x)=\eta_{\mu \nu}-\frac{x_{\mu} x_{\nu}}{x_{d}^{2}}, \\
& g^{\mu \nu}(x)=\eta^{\mu \nu}+x^{\mu} x^{\nu}, \\
& \Gamma_{\mu \nu}^{\rho}(x)=-g_{\mu \nu}(x) x^{\rho} .
\end{aligned}
$$

This gives:

$$
\nabla_{\mu} \partial_{\nu} x^{0}=\nabla_{\mu} \eta_{\nu}^{0}=-\Gamma_{\mu \nu}^{\rho} \eta_{\rho}^{0}=-\Gamma_{\mu \nu}^{0}=g_{\mu \nu} x^{0}
$$

i.e. 7.61) holds as announced. This shows that, for each $j=0, \ldots d$ the vector field $w_{\mu}=\partial_{\mu} x^{j}$ is a conformal-Killing vector field. 


\section{Positive quantization of tachyons fully breaking the de Sitter symmetry}

In this final section we construct all the positive quantizations of the tachyons and show that, for every real $\lambda$ including the integers, it is possible to write two-point functions which satisfy the Klein-Gordon equation with squared mass $m_{\lambda}^{2}$ and the canonical commutation relations, and possess the positive semi-definiteness property, but are not de Sitter invariant. This is possible because of the existence of compact spacelike sections in the de Sitter space-time which reduces the infrared problem to taking care of a finite number of degrees of freedom. Positivity may be restored while keeping locality at the price of breaking the de Sitter invariance. Several authors look at this as a welcome or else an unavoidable feature of the physical world. Others think that it is only a gauge artefact.

As before we assume that $\operatorname{Re} \lambda+(d-1) / 2>0, l$ and $n$ are non-negative integers, $x=(t, \mathbf{x})$, $z=i \operatorname{sh} t, x^{\prime}=\left(t^{\prime}, \mathbf{x}^{\prime}\right), z^{\prime}=i \operatorname{sh} t^{\prime}$. Suppose $\lambda$ is not a non-negative integer. The most general distribution $F$ on $X_{d} \times X_{d}$ satisfying the Klein-Gordon equation with squared mass $m_{\lambda}^{2}$ has the form

$$
\begin{aligned}
F_{\lambda}\left(x, x^{\prime}\right) & =\sum_{l M} F_{\lambda l M}\left(x, x^{\prime}\right)=(\operatorname{ch} t)^{-\kappa}\left(\operatorname{ch} t^{\prime}\right)^{-\kappa} \sum_{l M} V_{l M}\left(\mathbf{x}, \mathbf{x}^{\prime}\right)\left[A \mathbf{P}_{\lambda+\kappa}^{-l-\kappa}(z) \mathbf{P}_{\lambda+\kappa}^{-l-\kappa}\left(-z^{\prime}\right)+\right. \\
& \left.+B \mathbf{P}_{\lambda+\kappa}^{-l-\kappa}(-z) \mathbf{P}_{\lambda+\kappa}^{-l-\kappa}\left(z^{\prime}\right)+E \mathbf{P}_{\lambda+\kappa}^{-l-\kappa}(z) \mathbf{P}_{\lambda+\kappa}^{-l-\kappa}\left(z^{\prime}\right)+G \mathbf{P}_{\lambda+\kappa}^{-l-\kappa}(-z) \mathbf{P}_{\lambda+\kappa}^{-l-\kappa}\left(-z^{\prime}\right)\right] .
\end{aligned}
$$

The coefficients $A, B, E, G$ may depend on $\lambda, l$ and $M$. Imposing the canonical commutation relations 3.27) and (3.29) requires that

$$
A-B=\gamma_{l}(\lambda)
$$

We now assume $\lambda, t$ and $t^{\prime}$ are real. Then $\mathbf{P}_{\lambda+\kappa}^{-l-\kappa}(-z)=\overline{\mathbf{P}_{\lambda+\kappa}^{-l-\kappa}(z)}$ (see Eq. 3.15) and the condition for $F_{\lambda l M}\left(x, x^{\prime}\right)$ to be positive semi-definite is that

$$
A \geq 0, \quad B \geq 0, \quad G=\bar{E}, \quad|E|^{2} \leq A B .
$$

The normalization factor $\gamma_{l}(\lambda)$ is always positive for standard theories with $m_{\lambda}^{2}$ positive, while it may diverge and/or become negative in the tachyonic region of the spectrum (see figure 1). Always supposing that $\lambda$ is not a non-negative integer, let $\varepsilon$ be the sign of $\gamma_{l}(\lambda)$ i.e. $\varepsilon= \pm 1, \varepsilon \gamma_{l}(\lambda)>0$. The above conditions can be satisfied by taking

$$
\begin{array}{llll}
A=\gamma_{l}(\lambda) \operatorname{ch}^{2} \alpha_{l}, & B=\gamma_{l}(\lambda) \operatorname{sh}^{2} \alpha_{l}, & E=\bar{G}=\gamma_{l}(\lambda) \rho_{l} e^{-i \beta_{l}} \operatorname{ch} \alpha_{l} \operatorname{sh} \alpha_{l} & \text { if } \varepsilon=1 \\
A=-\gamma_{l}(\lambda) \operatorname{sh}^{2} \alpha_{l}, & B=-\gamma_{l}(\lambda) \operatorname{ch}^{2} \alpha_{l}, & E=\bar{G}=-\gamma_{l}(\lambda) \rho_{l} e^{i \beta_{l}} \operatorname{ch} \alpha_{l} \operatorname{sh} \alpha_{l} & \text { if } \varepsilon=-1,
\end{array}
$$

with real $\alpha_{l}$ and $\beta_{l}$, and $0 \leq \rho_{l} \leq 1$. In general the constants $\alpha_{l}, \beta_{l}$, and $\rho_{l}$ may depend on $\lambda$ and $M$, but we omit this to simplify the notations. This gives

$$
\begin{aligned}
& F_{\lambda l M}\left(x, x^{\prime}\right)=(\operatorname{ch} t)^{-\kappa}\left(\operatorname{ch} t^{\prime}\right)^{-\kappa} V_{l M}\left(\mathbf{x}, \mathbf{x}^{\prime}\right) \times \\
& \times\left[\varepsilon \gamma_{l}(\lambda)\left(\operatorname{ch} \alpha_{l} \mathbf{P}_{\lambda+\kappa}^{-l-\kappa}(\varepsilon z)+e^{i \beta_{l}} \operatorname{sh} \alpha_{l} \mathbf{P}_{\lambda+\kappa}^{-l-\kappa}(-\varepsilon z)\right)\left(\operatorname{ch} \alpha_{l} \mathbf{P}_{\lambda+\kappa}^{-l-\kappa}\left(-\varepsilon z^{\prime}\right)+e^{-i \beta_{l}} \operatorname{sh} \alpha_{l} \mathbf{P}_{\lambda+\kappa}^{-l-\kappa}\left(\varepsilon z^{\prime}\right)\right)\right. \\
& \left.-\varepsilon \gamma_{l}(\lambda)\left(1-\rho_{l}\right) \operatorname{ch} \alpha_{l} \operatorname{sh} \alpha_{l}\left(e^{-i \beta_{l}} \mathbf{P}_{\lambda+\kappa}^{-l-\kappa}(\varepsilon z) \mathbf{P}_{\lambda+\kappa}^{-l-\kappa}\left(\varepsilon z^{\prime}\right)+e^{i \beta_{l}} \mathbf{P}_{\lambda+\kappa}^{-l-\kappa}(-\varepsilon z) \mathbf{P}_{\lambda+\kappa}^{-l-\kappa}\left(-\varepsilon z^{\prime}\right)\right)\right] .
\end{aligned}
$$

This formula encodes every possible canonical quantization of the de Sitter scalar tachyons for noninteger real $\lambda$. The de Sitter symmetry is fully broken in the sense that there the corresponding Hilbert spaces do not contain any invariant subspace.

Let us now consider the case $\lambda \rightarrow n$. We show that the parameters $\alpha_{l}(\lambda), \beta_{l}(\lambda)$ and $\rho_{l}(\lambda)$ can be chosen as functions of $\lambda$ in such a manner that all $F_{\lambda l M}\left(x, x^{\prime}\right)$ have finite limits when $\lambda \rightarrow n$. We obtain in this way canonical quantum fields corresponding to any real value of $\lambda$ - including the integers. A similar limiting procedure has been described for the massless case $n=0$ in [22].

When $l>n$ there is nothing to do: the normalization factor $\gamma_{l}(\lambda)>0$ is continuous in a small real neighborhood $\omega$ of $n$. We can then fix $\alpha_{l}, \beta_{l}$, and $\rho_{l}$ in $\omega$ independently of $\lambda \in \omega$, and define $F_{n l M}$ by the formula (8.5) with $\lambda=n$ and $\varepsilon=1$. 
To discuss the case $l \leq n$ we need the general identities ([42, p. 144])

$$
\begin{aligned}
& \mathbf{P}_{\nu}^{\mu}(-z)=\mathbf{P}_{\nu}^{\mu}(z) \cos \pi(\mu+\nu)-\frac{2}{\pi} \mathbf{Q}_{\nu}^{\mu}(z) \sin \pi(\mu+\nu), \\
& \mathbf{Q}_{\nu}^{\mu}(-z)=-\mathbf{Q}_{\nu}^{\mu}(z) \cos \pi(\mu+\nu)-\frac{\pi}{2} \mathbf{P}_{\nu}^{\mu}(z) \sin \pi(\mu+\nu) .
\end{aligned}
$$

which imply, for $l \leq n$ (but not for $l>n$ ):

$$
\mathbf{P}_{n+\kappa}^{-l-\kappa}(z)=(-1)^{n-l} \mathbf{P}_{n+\kappa}^{-l-\kappa}(-z), \quad \mathbf{Q}_{n+\kappa}^{-l-\kappa}(z)=(-1)^{n-l+1} \mathbf{Q}_{n+\kappa}^{-l-\kappa}(-z) .
$$

Taking $\lambda$ real with $0<s=|\lambda-n|<1$, we will let $\lambda$ tend to $n$ keeping the sign of $\lambda-n$ fixed. For every $l \leq n$ we denote

$$
\varepsilon=\operatorname{sign}\left((-1)^{n-l+1}(\lambda-n)\right), \quad \lambda=n+\varepsilon \tau s, \quad \tau=(-1)^{n-l+1} .
$$

(this notation omits, for simplicity, the $l$ dependence of $\tau$ and $\varepsilon$ ). Then

$$
0<\varepsilon \gamma_{l}(\lambda)=\frac{\pi \Gamma(1+l+n+2 \kappa+\varepsilon \tau s)}{2 \sin (\pi s) \Gamma(1+n-l+\varepsilon \tau s)} \sim \frac{\Gamma(1+l+n+2 \kappa)}{2(n-l) ! s} \quad \text { as } \quad s \rightarrow 0 .
$$

We choose:

$$
e^{\alpha_{l}}=\frac{1}{A \sqrt{s}}, \quad \beta_{l}=(n-l+1) \pi-2 A B s, \quad \text { hence } \quad e^{i \beta_{l}}=\tau e^{-2 i A B s}, \quad \rho_{l}=e^{-2 A^{2} C s^{2}} .
$$

Here $A>0, B \in \mathbf{R}$, and $C \geq 0$ are (new) constants which again may depend on $l$ and also on $M$. By using (8.6) we get

$$
\begin{aligned}
& \operatorname{ch}\left(\alpha_{l}\right) \mathbf{P}_{\lambda+\kappa}^{-l-\kappa}(\varepsilon z)+e^{i \beta_{l}} \operatorname{sh}\left(\alpha_{l}\right) \mathbf{P}_{\lambda+\kappa}^{-l-\kappa}(-\varepsilon z)= \\
& =\left[\operatorname{ch}\left(\alpha_{l}\right)-e^{-2 i A B s} \operatorname{sh}\left(\alpha_{l}\right) \cos \pi(s)\right] \mathbf{P}_{n+\varepsilon \tau s+\kappa}^{-l-\kappa}(\varepsilon z)+\frac{2}{\pi} e^{-2 i A B s} \operatorname{sh}\left(\alpha_{l}\right) \sin \pi(\varepsilon \tau s) \mathbf{Q}_{n+\varepsilon \tau s+\kappa}^{-l-\kappa}(\varepsilon z) \\
& \sim \sqrt{s}\left[(A+i B) \mathbf{P}_{n+\kappa}^{-l-\kappa}(\varepsilon z)-\frac{\varepsilon(-1)^{n-l}}{A} \mathbf{Q}_{n+\kappa}^{-l-\kappa}(\varepsilon z)+\mathrm{O}(s)\right]
\end{aligned}
$$

when $s \rightarrow 0$. The behavior of the last term in 8.5) as $s \rightarrow 0$ is straightforward. Finally

$$
\begin{aligned}
& F_{n l M}\left((t, \mathbf{x}),\left(t^{\prime}, \mathbf{x}^{\prime}\right)\right)=\lim _{s \rightarrow 0} F_{\lambda l M}\left((t, \mathbf{x}),\left(t^{\prime}, \mathbf{x}^{\prime}\right)\right)= \\
& =\frac{\Gamma(1+l+n+2 \kappa)}{2(n-l) !}(\operatorname{ch} t)^{-\kappa}\left(\operatorname{ch} t^{\prime}\right)^{-\kappa}\{ \\
& {\left[(A+i B) \mathbf{P}_{n+\kappa}^{-l-\kappa}(z)-\frac{(-1)^{n-l}}{A} \mathbf{Q}_{n+\kappa}^{-l-\kappa}(z)\right]\left[(A-i B) \mathbf{P}_{n+\kappa}^{-l-\kappa}\left(-z^{\prime}\right)-\frac{(-1)^{n-l}}{A} \mathbf{Q}_{n+\kappa}^{-l-\kappa}\left(-z^{\prime}\right)\right]} \\
& \left.+C \mathbf{P}_{n+\kappa}^{-l-\kappa}(z) \mathbf{P}_{n+\kappa}^{-l-\kappa}\left(-z^{\prime}\right)\right\} Y_{l M}(\mathbf{x}) Y_{l M}\left(\mathbf{x}^{\prime}\right), \quad z=i \operatorname{sh} t, \quad z^{\prime}=i \operatorname{sh} t^{\prime} .
\end{aligned}
$$

We have repeatedly used (8.8). If the constants $A, B$, and $C$ have been chosen independently of $\varepsilon$, the limit $F_{n l M}$ is independent of $\varepsilon$. For real $t$ and $t^{\prime}, \mathbf{P}_{n+\kappa}^{-l-\kappa}\left(-z^{\prime}\right)=\overline{\mathbf{P}_{n+\kappa}^{-l-\kappa}\left(z^{\prime}\right)}$ and $\mathbf{Q}_{n+\kappa}^{-l-\kappa}\left(-z^{\prime}\right)=$ $\overline{\mathbf{Q}_{n+\kappa}^{-l-\kappa}\left(z^{\prime}\right)}$, so that $F_{n l M}\left(x, x^{\prime}\right)$ is a positive semi-definite kernel, as it must be as the limit of the positive semi-definite kernel $F_{\lambda l M}\left(x, x^{\prime}\right)$. Again by using (8.8) we obtain

$$
\begin{aligned}
& F_{n l M}\left(x, x^{\prime}\right)-F_{n l M}\left(x^{\prime}, x\right)=C_{n l M}\left(x, x^{\prime}\right)= \\
& \frac{\Gamma(1+l+n+2 \kappa)}{(n-l) !}(\operatorname{ch} t)^{-\kappa}\left(\operatorname{ch} t^{\prime}\right)^{-\kappa}\left[\mathbf{P}_{n+\kappa}^{-l-\kappa}(z) \mathbf{Q}_{n+\kappa}^{-l-\kappa}\left(z^{\prime}\right)-\mathbf{P}_{n+\kappa}^{-l-\kappa}\left(z^{\prime}\right) \mathbf{Q}_{n+\kappa}^{-l-\kappa}(z)\right] Y_{l M}(\mathbf{x}) Y_{l M}\left(\mathbf{x}^{\prime}\right) .
\end{aligned}
$$

This last expression, as expected, is independent of $A, B, C, \varepsilon$ and $M$. It gives an explicit form for $C_{n l M}\left(x, x^{\prime}\right)=\left.C_{\lambda l M}\left(x, x^{\prime}\right)\right|_{\lambda=n}, l \leq n$.

Therefore the general solution depends on three constants $A, B$, and $C$ (for each $l M$ with $l \leq n$ ) that are only required to be real with $A>0$ and $C \geq 0$. Making an arbitrary choice for each $l M$ produces an $F_{n l M}\left(x, x^{\prime}\right)$ given by (8.13) for $l \leq n$, and by (8.5) (with $\lambda=n$ and $\varepsilon=1$ ) for $l>n$. Under mild conditions the series $F_{n}\left(x, x^{\prime}\right)=\sum_{l M} F_{n l M}\left(x, x^{\prime}\right)$ will converge to a distribution with the following properties: 
1. $\left(\square_{x}+n(n+d-1)\right) F_{n}\left(x, x^{\prime}\right)=\left(\square_{x^{\prime}}+n(n+d-1)\right) F_{n}\left(x, x^{\prime}\right)=0$,

2. $F_{n}\left(x, x^{\prime}\right)$ is a positive semi-definite kernel,

3. $F_{n}\left(x, x^{\prime}\right)-F_{n}\left(x^{\prime}, x\right)=C_{n}\left(x, x^{\prime}\right)$.

Therefore $F_{n}\left(x, x^{\prime}\right)$ can be taken as the two-point function of a free field with squared mass $m_{n}^{2}=$ $-n(n+d-1)$, which however is not Lorentz invariant and is not maximally analytic. Moreover $F_{n}\left(x, x^{\prime}\right)$ is the limit from $\lambda>n$ and from $\lambda<n$ of two-point functions with similar properties.

A particularly simple choice is to let the two-point function $F_{n l M}$ coincide, for $l>n$, with the covariant maximal analytic $\widehat{W}_{n l M}$ i.e. to choose the infrared regular part of the two-point function according to the Bunch-Davies prescription (4.28) :

$$
\begin{aligned}
& F_{n l M}\left(x, x^{\prime}\right)=\mathcal{W}_{n l M}\left(x, x^{\prime}\right)=\widehat{\mathcal{W}}_{n l M}\left(x, x^{\prime}\right)= \\
& =\gamma_{n}(l)(\operatorname{ch} t)^{-\kappa}\left(\operatorname{ch} t^{\prime}\right)^{-\kappa} \mathbf{P}_{n+\kappa}^{-l-\kappa}(i \operatorname{sh} t) \mathbf{P}_{n+\kappa}^{-l-\kappa}\left(-i \operatorname{sh} t^{\prime}\right) Y_{l M}(\mathbf{x}) Y_{l M}\left(\mathbf{x}^{\prime}\right), \quad l>n .
\end{aligned}
$$

In particular Allen and Folacci's de Sitter breaking quantization of the massless scalar field and Folacci's quantization of the $n=1$ conformal field belong to this family. Whatever choice is made of the remaining part, the resulting $F_{n}$ cannot determine the same sesquilinear form on the space $\mathcal{E}_{n}$ as $\widehat{\mathcal{W}}_{n}$. Indeed if $f \in \mathcal{E}_{n}$, Eq. 6.31) implies that for $l \leq n$,

$\int_{X_{d} \times X_{d}} f(x) F_{n l M}\left(x, x^{\prime}\right) \overline{f\left(x^{\prime}\right)} d x d x^{\prime}=\frac{\Gamma(1+l+n+2 \kappa)}{2(n-l) !}\left|\int_{\mathbf{R}} \frac{1}{A}(\operatorname{ch} t)^{d-1-\kappa} \widetilde{f}_{l M}(t) \mathbf{Q}_{n+\kappa}^{-l-\kappa}(i \operatorname{sh} t) d t\right|^{2}$.

Since $\mathbf{P}_{n+\kappa}^{-l-\kappa}$ and $\mathbf{Q}_{n+\kappa}^{-l-\kappa}$ are linearly independent, it is possible to find (many) $\tilde{f}_{l M} \in \mathcal{D}(\mathbf{R})$ such that 6.31 holds while the rhs of 8.16) is not 0 . This points towards the physical inequivalence of the two constructions. The polynomials in the operators $\phi(f), f \in \mathcal{E}_{n}$, which can be regarded as the observable (gauge invariant) quantities, do not have the same expectation values in the two theories.

On the other hand let $f \in \mathcal{D}\left(X_{d}\right)$ satisfy $f(x)=(-1)^{n} f(-x)$. Since $Y_{l M}(-\mathbf{x})=(-1)^{l} Y_{l M}(\mathbf{x})$, it follows that $f_{l M}(-x)=(-1)^{n} f_{l M}(x)$ and hence $\widetilde{f}_{l M}(t)=(-1)^{n-l} \widetilde{f}_{l M}(-t)$. Since, for $l \leq n$, $\mathbf{Q}_{n+\kappa}^{-l-\kappa}(i \operatorname{sh} t)=(-1)^{n-l+1} \mathbf{Q}_{n+\kappa}^{-l-\kappa}(-i \operatorname{sh} t)$

$$
\int_{\mathbf{R}}(\operatorname{ch} t)^{d-1-\kappa} \widetilde{f}_{l M}(t) \mathbf{Q}_{n+\kappa}^{-l-\kappa}(i \operatorname{sh} t) d t=0 \quad \forall l \leq n .
$$

If we assume that $f$ also belongs to $\mathcal{E}_{n}$ we get from (8.16)

$$
\int_{X_{d} \times X_{d}} f(x) F_{n l M}\left(x, x^{\prime}\right) \overline{f\left(x^{\prime}\right)} d x d x^{\prime}=0 \quad \forall l \leq n .
$$

Let us suppose that the choice [8.15) has been made, i.e. $F_{n l M}=\widehat{\mathcal{W}}_{n l M}$ for all $l>n$. Then $F_{n}$ and $\widehat{\mathcal{W}}_{n}$ define the same (Lorentz invariant) sesquilinear form on the subspace

$$
\mathcal{F}_{n}=\left\{f \in \mathcal{E}_{n}: f(x)=(-1)^{n} f(-x) \quad \forall x \in X_{d}\right\} .
$$

In this particular case, the Hilbert space determined by the non-invariant sesquilinear form $F_{n}$ nevertheless contains a subspace equipped with a unitary representation of the Lorentz group. Let $\mathcal{N}$ denote the null-space of $\widehat{\mathcal{W}}_{n}$ as a positive-semi-definite sesquilinear form on $\mathcal{E}_{n}$. According to Lemma 6.4 the map $f \mapsto \widetilde{f}_{1-d-n,+}$ defines an injective map of $\mathcal{E}_{n} / \mathcal{N}$ into $\widetilde{\mathcal{E}}_{n}^{(2)}$. It is easy to find elements $f$ of $\mathcal{F}_{n}$ such that $\widetilde{f}_{1-d-n,+} \neq 0$. On the completion of $\widetilde{\mathcal{E}}_{n}^{(2)}$ (with respect to the scalar product (6.17) , the natural representation of the Lorentz group is irreducible and belongs to the discrete series with squared mass $m_{n}^{2}$ (see [41, chap. X], [31]). Therefore the completions of the images of $\mathcal{E}_{n}$ and $\mathcal{F}_{n}$ under the same map must be the same, i.e. the whole completion of $\widetilde{\mathcal{E}}_{n}^{(2)}$. The sesquilinear form $\widehat{\mathcal{W}}_{n}$ determines (by the usual quotienting and completion process) the same Hilbert space, whether one starts from $\mathcal{E}_{n}$ or $\mathcal{F}_{n}$, and the sesquilinear form $F_{n}$ determines the same Hilbert space starting from $\mathcal{F}_{n}$. The unitary representation of the Lorentz group acting there is irreducible with squared mass $m_{n}^{2}$.

\section{Acknowledgements}

We thank Jacques Bros, Antoine Folacci and Richard Woodard for discussions and correspondence. U. M. thanks the IHES for their kind hospitality. 


\section{A Appendix. Gegenbauer polynomials and hyperspher- ical harmonics}

This appendix follows [40, Chap. XI], 41, Chap. IX], and [59, Chap. IV], to which we refer the reader for more details. For a positive integer $n$ and a complex $\nu$, the Gegenbauer polynomial $C_{n}^{\nu}$ is defined as

$$
\begin{aligned}
C_{n}^{\nu}(z) & =\frac{\Gamma(n+2 \nu)}{\Gamma(n+1) \Gamma(2 \nu)} F\left(-n, n+2 \nu ; \nu+\frac{1}{2} ; \frac{1-z}{2}\right) \\
& =\frac{2^{\frac{1}{2}-\nu} \Gamma\left(\frac{1}{2}\right) \Gamma(n+2 \nu)\left(1-z^{2}\right)^{\frac{1}{4}-\frac{\nu}{2}}}{\Gamma(n+1) \Gamma(\nu)} \mathbf{P}_{n-\frac{1}{2}+\nu}^{\frac{1}{2}-\nu}(z)
\end{aligned}
$$

Here $\mathbf{P}_{\nu}^{\mu}$ is the "Legendre function on the cut" mentioned in Sect. 3. Also:

$$
\sum_{n=0}^{\infty} w^{n} C_{n}^{\nu}(z)=\left(1-2 z w+w^{2}\right)^{-\nu}=\sum_{n=0}^{\infty}(-w)^{n} C_{n}^{\nu}(-z), \quad C_{n}^{\nu}(-z)=(-1)^{n} C_{n}^{\nu}(z) .
$$

As a consequence of this, if $\tau, z \in \mathbf{C}, \tau^{n} C_{n}^{\nu}(z / \tau)$ is a homogeneous polynomial of degree $n$ in $\tau$ and $z$ and is even in $\tau$, so that it is a polynomial in $z$ and $\tau^{2}$. In particular $C_{0}^{\nu}(z)=1$ and $C_{1}^{\nu}(z)=2 \nu z$.

Let $E_{N}$ denote the $N$-dimensional Euclidean space equipped with the scalar product $x \cdot y=$ $\sum_{j=1}^{N} x_{j} y_{j}$ and $S^{N-1}$ the unit sphere in $E_{N}$ equipped with the measure $d \sigma(x)=2 \delta(1-x$. $x) d x \ldots d x_{N}$, with total measure $\Omega_{N}=2 \pi^{N / 2} / \Gamma(N / 2)$. We suppose $N \geq 2$. We denote $e_{j}$ the vector with components $e_{j k}=\delta_{j k}$. A function $f$ on $E_{N}$ is called harmonic if $\Delta f=0$. For a fixed integer $l \geq 0$, the vector space $\mathcal{H}^{N, l}$ of complex harmonic polynomials homogeneous of degree $l$ on $E_{N}$ has dimension

$$
h(N, l)=\frac{(2 l+N-2)(N+l-3) !}{(N-2) ! l !} .
$$

$\mathcal{H}^{N, l}$ is a Hilbert space with the scalar product

$$
(f, g)=\int_{S^{N-1}} \overline{f(x)} g(x) d \sigma(x),
$$

and the natural (quasi-regular) representation of the rotation group of $E_{N}$ reduces to a unitary

irreducible (for $N \geq 3$ ) representation on $\mathcal{H}^{N, l}$. An orthonormal basis of $\mathcal{H}^{N, l}$ is called a system of hyperspherical harmonics. For any such basis $\left\{S_{j}\right\}_{1 \leq j \leq h(N, l)}$ the following identity holds for $\mathbf{x}, \mathbf{y} \in S^{N-1}$ (40, 11.4 (2). p. 243]):

$$
\sum_{j=1}^{h(N, l)} S_{j}^{*}(\mathbf{x}) S_{j}(\mathbf{y})=\sum_{j=1}^{h(N, l)} S_{j}(\mathbf{x}) S_{j}^{*}(\mathbf{y})=\frac{(2 l+N-2) \Gamma(N / 2)}{2 \pi^{N / 2}(N-2)} C_{l}^{\frac{N-2}{2}}(\mathbf{x} \cdot \mathbf{y})
$$

The corresponding identity for $x \cdot x \neq 1$ and $y \cdot y \neq 1$ is obtained by homogeneity by applying the above formula to $x / \sqrt{x \cdot x}$ and $y / \sqrt{y \cdot y}$ and using $S_{j}(x)=(x \cdot x)^{l / 2} S_{j}(x / \sqrt{x \cdot x})$. In particular, denoting $r=\sqrt{x \cdot x}$ and $s=\sqrt{y \cdot y}$

$$
r^{l} s^{l} C_{l}^{\frac{N-2}{2}}(x \cdot y / r s)
$$

is a homogeneous polynomial in $x, y, r, s$ (since it is even in $r$ and in $s$ as remarked above), harmonic in $x$ and in $y$.

A particular orthonormal basis $\left\{\mathbf{Y}_{K}^{N, l}\right\}$ of $\mathcal{H}^{N, l}$ is labelled by the multi-indices $K$ of the form

$$
K=\left(k_{1}, \ldots, \pm k_{N-2}\right), \quad l=k_{0} \geq k_{1} \geq \ldots \geq k_{N-2} \geq 0
$$

where the $k_{j}$ are integers:

$$
\mathbf{Y}_{K}^{N, l}(x)=A_{K}^{N, l} \prod_{j=0}^{N-3} r_{N-j}^{k_{j}-k_{j+1}} C_{k_{j}-k_{j+1}}^{\frac{N-j-2}{2}+k_{j+1}}\left(\frac{x_{N-j}}{r_{N-j}}\right) \times\left(x^{\prime} \pm i x\right)^{k_{N-2}}
$$


Here $r_{k}^{2}=x^{2}+\ldots+x_{k}^{2}$. We will rather use a real basis $\left\{Y_{K}^{N, l}\right\}$ given by

$$
\begin{aligned}
& Y_{K}^{N, l}=\mathbf{Y}_{K}^{N, l} \quad \text { if } k_{N-2}=0, \\
& Y_{\left(k_{1}, \ldots,+k_{N-2}\right)}^{N, l}=\frac{1}{\sqrt{2}} \mathbf{Y}_{\left(k_{1}, \ldots,+k_{N-2}\right)}^{N, l}+\frac{1}{\sqrt{2}} \mathbf{Y}_{\left(k_{1}, \ldots,-k_{N-2}\right)}^{N, l} \text { if } \quad k_{N-2}>0, \\
& Y_{\left(k_{1}, \ldots,-k_{N-2}\right)}^{N, l}=\frac{1}{i \sqrt{2}} \mathbf{Y}_{\left(k_{1}, \ldots,+k_{N-2}\right)}^{N, l}-\frac{1}{i \sqrt{2}} \mathbf{Y}_{\left(k_{1}, \ldots,-k_{N-2}\right)}^{N, l} \quad \text { if } k_{N-2}>0 .
\end{aligned}
$$

The positive constants $A_{K}^{N, l}$ ensure the orthonormality of these bases. For $l=0, Y_{0}^{N, 0}$ is the constant $\Omega_{N}^{-1 / 2}$. For $l>0$, in the case $K=0$ (i.e. $k_{j}=0$ for all $j \geq 1$ ) $Y_{0}^{N, l}=\mathbf{Y}_{0}^{N, l}$ depends only on $x_{N}$ and $r_{N}$, and $Y_{K}^{N, l}\left(e_{N}\right)=0$ if $K \neq 0$. In the case $l=1, K$ can take the values $K_{0}, \ldots, K_{N-1}$ where $K_{0}=(0, \ldots, 0)$. $K_{1}=(1,0, \ldots 0)$, etc., $K_{N-2}=(1, \ldots,+1), K_{N-1}=(1, \ldots,-1)$. It is clear that $Y_{K_{j}}^{1}(x)$ is proportional to $x_{N-j}$ and the constant factor is determined by the orthonormality property, so that, finally, $Y_{K_{j}}^{1}(x)=\left(N / \Omega_{N}\right)^{1 / 2} x_{N-j}, 1 \leq j \leq N$. In this case we may abbreviate the notation $Y_{K_{j}}^{1}$ to $Y_{N-j}^{1}$.

Let $K$ be a multi-index as in (A.8), $m$ and $l$ be integers such that $m \geq l \geq k_{1}$ and $L=(l, K)$ be the multi-index in $N+1$ variables given by

$$
L=\left(l_{0}=m, l_{1}=l, \ldots, l_{j}=k_{j-1}, \ldots, \pm l_{N-1}= \pm k_{N-2}\right) .
$$

Then

$$
\mathbf{Y}_{(l, K)}^{N+1, m}\left(\left(x, \ldots, x_{N+1}\right)\right)=\frac{A_{(l, K)}^{N+1, m}}{A_{K}^{N, l}} r_{N+1}^{m-l} C_{m-l}^{\frac{N-1}{2}+l}\left(\frac{x_{N+1}}{r_{N+1}}\right) \mathbf{Y}_{K}^{l}\left(\left(x, \ldots, x_{N}\right)\right)
$$

The same holds for the corresponding $Y$.

Let us choose $N=d$. Let $E_{d+1}^{(c)}$ be the complexified of $E_{d+1}$, i.e. $\mathbf{C}^{d+1}$ equipped with the same scalar product as $E_{d+1}$. Let $J$ be the bijection of $M_{d+1}^{(c)}$ onto $E_{d+1}^{(c)}$ defined by

$$
\begin{gathered}
(J x)_{d+1}=i x^{0}, \quad(J x)_{j}=x^{j} \quad \text { for } 1 \leq j \leq d, \\
\left.(J x \cdot J y)\right|_{E_{d+1}^{(c)}}=-\left.(x \cdot y)\right|_{M_{d+1}^{(c)}} .
\end{gathered}
$$

If $p$ is a homogeneous pseudo-harmonic polynomial on $M_{d+1}$ (i.e. $\square_{\text {Mink }} p=0$ ), it extends to a homogeneous pseudo-harmonic polynomial on $M_{d+1}^{(c)}$ and $x \mapsto p\left(J^{-1} x\right)$ restricted to $E_{d+1}$ is a homogeneous harmonic polynomial on $E_{d+1}$. Thus, by analytic continuation, we find that the space $\underline{\mathcal{H}^{d+1, m}}$ of homogeneous pseudo-harmonic polynomials of degree $m$ on $M_{d+1}$ is of dimension $h(N+1, m)$ and carries an irreducible representation of the Lorentz group on $M_{d+1}$. A basis of this space is provided by

$$
Y_{L}^{d+1, m}\left(x^{1}, \ldots, x^{d}, i x^{0}\right)
$$

Rewriting $L$ as $(l, K)=\left(l, k_{1}, \ldots, \pm k_{d-2}\right)$ with $m \geq l \geq k_{1} \geq \ldots \geq k_{d-2} \geq 0$, it follows that a basis of $\underline{\mathcal{H}^{N+1, m}}$ is provided by the polynomials

$$
\underline{Y_{L}^{d+1, m}}\left(x^{0}, \vec{x}\right)=\frac{A_{(l, K)}^{N+1, m}}{A_{K}^{N, l}} i^{l-m}(-(x \cdot x))^{\frac{m-l}{2}} C_{m-l}^{\frac{d-1}{2}+l}\left(\frac{i x^{0}}{(-(x \cdot x))^{1 / 2}}\right) Y_{K}^{l}(\vec{x}) .
$$

As noted before, this is a real polynomial in $x$, whatever the determination chosen for $(-(x \cdot x))^{1 / 2}$.

\section{B Appendix. Expansion of plane waves into hyper- spherical harmonics}

In this appendix we derive the expansion of the de Sitter plane wave $z \mapsto(z \cdot \xi)^{\lambda}$ into hyperspherical harmonics, where $z \in \mathcal{T}_{ \pm}$and $\xi \in C_{+} \backslash\{0\}$ (see (4.25)). We also derive a similar expansion for $\left(\xi, \xi^{\prime}\right) \mapsto\left(\xi \cdot \xi^{\prime}\right)^{\lambda}$, where both $\xi$ and $\xi^{\prime}$ belong to $C_{+} \backslash\{0\}$.

For $x \in X_{d}$ and $\xi \in S_{0}$ we use the coordinates $x=x(t, \mathbf{x})$ and $\xi=\xi(\boldsymbol{\xi})$ given in (2.7) and (2.10). 


\section{B.1 Waves}

The representation

$$
a^{\lambda}=\frac{1}{\Gamma(-\lambda)} \int_{0}^{\infty} \frac{d u}{u} u^{-\lambda} e^{-u a} .
$$

is valid for $\operatorname{Re} \lambda<0$ and $\operatorname{Re} a>0$. Let $x+i y \in \mathcal{T}_{+}$and $\xi \in S_{0}$. Then

$$
\operatorname{Re}(-i(x+i y) \cdot \xi)=y \cdot \xi>0,
$$

so that

$$
((x+i y) \cdot \xi)^{\lambda}=\frac{e^{\frac{i \pi \lambda}{2}}}{\Gamma(-\lambda)} \int_{0}^{\infty} \frac{d u}{u} u^{-\lambda} e^{i u(x+i y) \cdot \xi} .
$$

If we consider a purely imaginary event such as

$$
x\left(t+\frac{i \pi}{2}, \mathbf{x}\right)=\left\{\begin{array}{l}
x^{0}=\operatorname{sh}\left(t+\frac{i \pi}{2}\right)=i \operatorname{ch} t \\
\vec{x}=\operatorname{ch}\left(t+\frac{i \pi}{2}\right) \mathbf{x}=i \operatorname{sh} t \mathbf{x}
\end{array}\right.
$$

we get

$$
\left[x\left(t+\frac{i \pi}{2}, \mathbf{x}\right) \cdot \xi(\boldsymbol{\xi})\right]^{\lambda}=\frac{e^{\frac{i \pi \lambda}{2}}}{\Gamma(-\lambda)} \int_{0}^{\infty} \frac{d u}{u} u^{-\lambda} e^{-u \operatorname{ch} t+u \operatorname{sh} t \mathbf{x} \cdot \boldsymbol{\xi}}
$$

Recall now the well-known Neumann expansion of the exponential plane wave in terms of Gegenbauer polynomials (see e.g. 40, 7.10 .1 (5) p. 64]) :

$$
e^{i \gamma z}=\left(-\frac{2}{z}\right)^{\nu} \Gamma(\nu) \sum_{l=0}^{\infty}(-i)^{l}(\nu+l) C_{l}^{\nu}(\gamma) J_{\nu+l}(-z) .
$$

By setting $\nu=\frac{d-2}{2}$ and taking advantage of the well-known expansion of $C_{l}^{\nu}(\mathbf{x} \cdot \boldsymbol{\xi})$ in terms of the hyperspherical harmonics (see Appendix $\mathrm{A}$ ):

$$
e^{u \operatorname{sh} t \mathbf{x} \cdot \boldsymbol{\xi}}=(2 \pi)^{\frac{d}{2}} \sum_{l=0}^{\infty} i^{l+\frac{d-2}{2}}(u \operatorname{sh} t)^{-\frac{d-2}{2}} J_{l+\frac{d-2}{2}}(i u \operatorname{sh} t) \sum_{M} Y_{l M}(\mathbf{x}) Y_{l M}(\boldsymbol{\xi}) .
$$

By inserting this expression in the above integral representation we get that

$$
\begin{aligned}
& {\left[x\left(t+\frac{i \pi}{2}, \mathbf{x}\right) \cdot \xi(\boldsymbol{\xi})\right]^{\lambda}=} \\
& =\frac{i^{\lambda}}{(2 \pi)^{\frac{d}{2}} \Gamma(-\lambda)(\operatorname{sh} t)^{\frac{d-2}{2}}} \sum_{l=0}^{\infty} \int_{0}^{\infty} \frac{d u}{u} u^{-\lambda-\frac{d-2}{2}} e^{-u \operatorname{ch} t} I_{l+\frac{d-2}{2}}(u \operatorname{sh} t) \sum_{M} Y_{l M}(\mathbf{x}) Y_{l M}(\boldsymbol{\xi})
\end{aligned}
$$

The integral at the RHS is the Mellin transform of a product that can be evaluated by the MellinBarnes integral. This is a way to directly check [40, 7.8 (9) p. 57] :

$$
\begin{aligned}
& \int_{0}^{\infty} \frac{d u}{u} u^{-\lambda-\frac{d-2}{2}} e^{-u \operatorname{ch} t} I_{l+\frac{d-2}{2}}(u \operatorname{sh} t)= \\
& =(\operatorname{sh} t)^{\lambda+\frac{d-2}{2}} \int_{0}^{\infty} \frac{d v}{v} v^{-\lambda-\frac{d-2}{2}} e^{-\frac{v \operatorname{ch} t}{\operatorname{sh} t}} I_{l+\frac{d-2}{2}}(v)=\Gamma(l-\lambda) P_{\lambda+\frac{d-2}{2}}^{-l-\frac{d-2}{2}}(\operatorname{ch} t) .
\end{aligned}
$$

Therefore

$$
\begin{aligned}
& {\left[x\left(t+\frac{i \pi}{2}, \mathbf{x}\right) \cdot \xi(\boldsymbol{\xi})\right]^{\lambda}=} \\
& =\frac{e^{\frac{i \pi \lambda}{2}}(2 \pi)^{\frac{d}{2}}}{\Gamma(-\lambda)\left(-i \operatorname{ch}\left(t+\frac{i \pi}{2}\right)\right)^{\frac{d-2}{2}}} \sum_{l=0}^{\infty} \Gamma(l-\lambda) P_{\lambda+\frac{d-2}{2}}^{-l-\frac{d-2}{2}}\left(-i \operatorname{sh}\left(t+\frac{i \pi}{2}\right)\right) \sum_{M} Y_{l M}(\mathbf{x}) Y_{l M}(\boldsymbol{\xi}) \\
& =\frac{(2 \pi)^{\frac{d}{2}}}{\Gamma(-\lambda)\left(\operatorname{ch}\left(t+\frac{i \pi}{2}\right)\right)^{\frac{d-2}{2}}} \sum_{l=0}^{\infty} \Gamma(l-\lambda) e^{\frac{i \pi(\lambda-l)}{2}} \mathbf{P}_{\lambda+\frac{d-2}{2}}^{-l-\frac{d-2}{2}}\left(-i \operatorname{sh}\left(t+\frac{i \pi}{2}\right)\right) \sum_{M} Y_{l M}(\mathbf{x}) Y_{l M}(\boldsymbol{\xi}) .
\end{aligned}
$$


By analytic continuation this remains valid if $t+i \pi / 2$ is replaced by any complex $t$ with $0<\operatorname{Im} t<\pi$, i.e. such that $x(t, \mathbf{x}) \in \mathcal{T}_{+}$. As a consequence of (B.10),

$$
\int_{S_{0}}(x(t, \mathbf{x}) \cdot \xi)^{\lambda} Y_{l M}(\boldsymbol{\xi}) d \boldsymbol{\xi}=\frac{e^{\frac{i \pi(\lambda-l)}{2}}(2 \pi)^{\frac{d}{2}} \Gamma(l-\lambda)}{\Gamma(-\lambda)(\operatorname{ch} t)^{\frac{d-2}{2}}} \mathbf{P}_{\lambda+\frac{d-2}{2}}^{-l-\frac{d-2}{2}}(-i \operatorname{sh} t) Y_{l M}(\mathbf{x})
$$

holds for all $l, M, 0<\operatorname{Im} t<\pi$, and $\lambda$ such that $\operatorname{Re} \lambda<0$. But since both sides of this equation are entire in $\lambda$, it remains valid for all $\lambda$. Since, moreover, any $C^{\infty}$ function on the sphere $S_{0} \simeq S^{d-1}$ has a convergent expansion into hyperspherical harmonics, the validity of (B.10) also extends to all $\lambda$.

\section{B.2 The kernel $\left(\xi \cdot \xi^{\prime}\right)^{\lambda}$}

Once again, if $-(d-1) / 2<\operatorname{Re} \lambda<0$,

$$
\left(\xi \cdot \xi^{\prime}\right)^{\lambda}=\frac{1}{\Gamma(-\lambda)} \int_{0}^{\infty} \frac{d u}{u} u^{-\lambda} e^{-u+u \xi \cdot \xi^{\prime}} .
$$

Again using (B.6), setting $\nu=(d-2) / 2$, and using the expansion of $C_{l}^{\nu}\left(\boldsymbol{\xi} \cdot \boldsymbol{\xi}^{\prime}\right)$ in terms of the hyperspherical harmonics, we obtain

$$
e^{u \boldsymbol{\xi} \cdot \boldsymbol{\xi}^{\prime}}=(2 \pi)^{\frac{d}{2}} \sum_{l=0}^{\infty} u^{-\frac{d-2}{2}} I_{l+\frac{d-2}{2}}(u) \sum_{M} Y_{l M}(\boldsymbol{\xi}) Y_{l M}\left(\boldsymbol{\xi}^{\prime}\right)
$$

Inserting this into the integral representation B.12 we get

$$
\left(\xi \cdot \xi^{\prime}\right)^{\lambda}=\frac{(2 \pi)^{\frac{d}{2}}}{\Gamma(-\lambda)} \sum_{l=0}^{\infty} \int_{0}^{\infty} \frac{d u}{u} u^{-\lambda-\frac{d-2}{2}} e^{-u} I_{l+\frac{d-2}{2}}(u) \sum_{M} Y_{l M}(\mathbf{x}) Y_{l M}(\boldsymbol{\xi}) .
$$

The integral can be evaluated by the Mellin-Barnes integral:

$$
\int_{0}^{\infty} \frac{d u}{u} u^{-\lambda-\frac{d-2}{2}} e^{-u} I_{l+\frac{d-2}{2}}(u)=2^{\frac{d}{2}+\lambda+l} \Gamma(l-\lambda) \pi^{-\frac{1}{2}} \frac{\Gamma\left(\frac{2 \lambda+d-1}{2}\right)}{\Gamma(\lambda+d+l-1)}
$$

Therefore

$$
\left(\xi \cdot \xi^{\prime}\right)^{\lambda}=\frac{(4 \pi)^{\frac{d-1}{2}}}{\Gamma(-\lambda)} \sum_{l=0}^{\infty} 2^{\lambda+l} \Gamma(l-\lambda) \frac{\Gamma\left(\frac{2 \lambda+d-1}{2}\right)}{\Gamma(\lambda+d+l-1)} \sum_{M} Y_{l M}(\boldsymbol{\xi}) Y_{l M}\left(\boldsymbol{\xi}^{\prime}\right) .
$$




\section{Appendix. Spaces of functions}

Lemma C.1 Let $N \geq 0$ be an integer and $\varphi$ be a $\mathcal{C}^{\infty}$ function on the sphere $S_{0}=C_{+} \cap\left\{\xi: \xi^{0}=1\right\}$, such that, for every $\vec{u} \in \mathbf{R}^{d}$,

$$
\int_{S_{0}}(\vec{u} \cdot \vec{\xi})^{N} \varphi(\vec{\xi}) d \vec{\xi}=0 .
$$

Then

$$
\int_{S_{0}} p(\vec{\xi}) \varphi(\vec{\xi}) d \vec{\xi}=0
$$

for every homogeneous polynomial $p$ of degree $N$ on $\mathbf{R}^{d}$.

Proof. Let $\alpha=\left(\alpha_{1}, \ldots, \alpha_{d}\right)$ be a multi-index with $|\alpha|=N$. Applying $(\partial / \partial \vec{u})^{\alpha}$ to the lhs of C.1 shows that $\varphi$ is orthogonal to the monomial $\vec{\xi}^{\alpha}$.

Lemma C.2 Let $\mu \in \mathbf{C}$ not be a non-negative integer, and $\varphi$ be a $\mathcal{C}^{\infty}$ function on the sphere $S_{0}$. Assume that, for all $z \in \mathcal{T}_{+}$(or for all $z \in \mathcal{T}_{-}$),

$$
\int_{S_{0}}(z \cdot \xi)^{\mu} \varphi(\xi) d \vec{\xi}=0 .
$$

Then $\varphi=0$.

Proof. If $z \in \mathcal{T}_{+}$, i.e. $z \in M_{d+1}, z \cdot z=-1$ and $\operatorname{Im} z \in V_{+}$, then (C.3) is equivalent to

$$
\int_{S_{0}}\left(1-\sum_{j=1}^{d} \frac{z_{j}}{z_{0}} \xi_{j}\right)^{\mu} \varphi(\xi) d \vec{\xi}=0 .
$$

Since $\left(z_{j} / z_{0}\right)$ are independent variables in $\mathcal{T}_{+}$, the condition (C.4) is equivalent (by analytic continuation) to the following:

$$
\int_{S_{0}}\left(1-\sum_{j=1}^{d} u_{j} \xi_{j}\right)^{\mu} \varphi(\xi) d \vec{\xi}=0
$$

for all complex $\left(u_{1}, \ldots, u_{d}\right)$ in the domain

$$
\left\{\left(u_{1}, \ldots, u_{d}\right) \in \mathbf{C}^{d}: \sum_{j=1}^{d}\left|u_{j}\right|^{2}<1\right\}
$$

Indeed this domain is convex, hence connected, and contains points in the image of $\mathcal{T}_{+}$under the map $z \mapsto \vec{z} / z_{0}$ (e.g. $z=i e_{0}$ ). Differentiating with respect to the $u_{j}$ and setting $u_{j}=0$ we find

$$
\int_{S_{0}} \xi^{\alpha} \varphi(\xi) d \vec{\xi}=0
$$

for any multi-power $\alpha$, hence $\varphi=0$. The same proof holds if $\mathcal{T}_{+}$is replaced by $\mathcal{T}_{-}$.

Lemma C.3 Let $N \geq 0$ be an integer, and $\varphi$ be a $\mathcal{C}^{\infty}$ function on the sphere $S_{0}$ such that

$$
\int_{S_{0}}(z \cdot \xi)^{N} \varphi(\xi) d \vec{\xi}=0
$$

for all $z \in C_{+}$. Then

$$
\int_{S_{0}} p(\vec{\xi}) \varphi(\xi) d \vec{\xi}=0
$$

for all polynomial $p$ of degree $\leq N$ on $\mathbf{R}^{d}$.

As a consequence of this lemma, $\mathcal{E}_{n}^{(1)} \subset \mathcal{E}_{n}^{(2)}$. Indeed if $f \in \mathcal{E}_{n}^{(1)}$, it satisfies (C.8) with $N=n$, hence (C.9), so that $f \in \mathcal{E}_{n}^{(2)}$. Therefore $\mathcal{E}_{n}^{(1)}=\mathcal{E}_{n}^{(2)}$.

Proof of Lemma C.3. By analytic continuation and homogeneity, (C.8) is equivalent to

$$
\int_{S_{0}}(1-\vec{u} \cdot \vec{\xi})^{N} \varphi(\vec{\xi}) d \vec{\xi}=0
$$


for all $\vec{u} \in \mathbf{C}^{d}$ such that $\sum_{j=1}^{d} u_{j}^{2}=1$. The assertion of the lemma is obvious if $N=0$. Assume it has been proved for $N<n$, where $n \geq 1$ is an integer. Let $\varphi$ be such that (C.10) holds for $N=n$. If $\vec{u}$ remains in an open set of the sphere where $u_{d} \neq 0$, we can take $u_{1}, \ldots, u_{d-1}$ as independent coordinates, with $u_{d}^{2}=1-\sum_{j=1}^{d-1} u_{j}^{2}$. Denoting $I_{N}$ the lhs of C.10 and differentiating it with respect to $u_{j},(1 \leq j \leq d-1)$, we find

$$
\frac{\partial}{\partial u_{j}} I_{n}=\int_{S_{0}} n\left(-\xi_{j}+\frac{u_{j} \xi_{d}}{u_{d}}\right)(1-\vec{u} \cdot \vec{\xi})^{n-1} \varphi(\vec{\xi}) d \vec{\xi}=0
$$

and hence

$$
\left(1-\frac{1}{n} \sum_{j=1}^{d-1} u_{j} \frac{\partial}{\partial u_{j}}\right) I_{n}=\int_{S_{0}}\left(1-\frac{\xi_{d}}{u_{d}}\right)(1-\vec{u} \cdot \vec{\xi})^{n-1} \varphi(\vec{\xi}) d \vec{\xi}=0
$$

There is nothing special about the index $d$, hence, for all $j$,

$$
\int_{S_{0}}\left(u_{j}-\xi_{j}\right)(1-\vec{u} \cdot \vec{\xi})^{n-1} \varphi(\vec{\xi}) d \vec{\xi}=0
$$

If $n=1$ this reduces to

$$
\int_{S_{0}}\left(u_{j}-\xi_{j}\right) \varphi(\vec{\xi}) d \vec{\xi}=0
$$

for $1 \leq j \leq d$. Taking $u_{j}=0$ shows that $\varphi$ is orthogonal to all homogeneous polynomials of degree 1 , then $I_{1}=0$ gives that $\int \varphi=0$. Suppose $n \geq 2$. Again taking $u_{1}, \ldots, u_{d-1}$ as independent coordinates, and differentiating (C.13) with respect to $u_{j}$ for $j<d$, we get

$$
\int_{S_{0}}\left(1-\vec{u} \cdot \vec{\xi}+(n-1)\left[-\left(u_{j} \xi_{j}\right)\left(1+\frac{\xi_{d}}{u_{d}}\right)+u_{j}^{2} \frac{\xi_{d}}{u_{d}}+\xi_{j}^{2}\right]\right)(1-\vec{u} \cdot \vec{\xi})^{n-2} \varphi(\vec{\xi}) d \vec{\xi}=0
$$

Summing this over $j<d$ gives

$$
\int_{S_{0}}\left(d-1+(n-1)\left(1+\frac{\xi_{d}}{u_{d}}\right)\right)(1-\vec{u} \cdot \vec{\xi})^{n-1} \varphi(\vec{\xi}) d \vec{\xi}=0 .
$$

Combining this with gives

$$
\int_{S_{0}}(1-\vec{u} \cdot \vec{\xi})^{n-1} \varphi(\vec{\xi}) d \vec{\xi}=0 .
$$

By the induction hypothesis, $\varphi$ is orthogonal to any polynomial in $\vec{\xi}$ of degree $<n$, hence, by expanding (C.10),

$$
\int_{S_{0}}(\vec{u} \cdot \vec{\xi})^{n} \varphi(\vec{\xi}) d \vec{\xi}=0 .
$$

By homogeneity this holds for all $\vec{u} \in \mathbf{C}^{d}$. By Lemma C.1 this implies that $\varphi$ is orthogonal to any homogeneous polynomial of degree $n$ in $\vec{\xi}$, and finally to any polynomial of degree $\leq n$. This completes the proof of Lemma C.3

We end this appendix with an explanation of the positivity of the kernel $\left(\vec{\xi} \cdot \overrightarrow{\xi^{\prime}}\right)^{p}$ on the unit sphere $S_{0}$. This is obvious for $p=1$, and, for $p>1$, it follows from the following classical result: the pointwise product of two positive-semi-definite kernels, if it is defined, is also positive-semi-definite. In the case of square matrices this product is the Schur product (see e.g. [58, 6.3.1, p.82]) and the same proof can be given in the following simple case:

Lemma C.4 Let $A\left(x, x^{\prime}\right)$ and $B\left(x, x^{\prime}\right)$ be two $\mathcal{C}^{\infty}$ functions with compact support on $V \times V$, where $V$ is a smooth manifold, $\mu$ a positive measure on $V$ with smooth density. Assume that $A$ and $B$ are positive-semi-definite, i.e. $\int_{V} \overline{f(x)} A\left(x, x^{\prime}\right) f\left(x^{\prime}\right) d \mu(x) d \mu\left(x^{\prime}\right) \geq 0$ for all smooth $f$ with compact support, and similarly for $B$. Then the same is true for $C\left(x, x^{\prime}\right)=A\left(x, x^{\prime}\right) B\left(x, x^{\prime}\right)$.

Proof (sketch). $A, B$ and $C$ are the kernels of Hilbert-Schmidt operators on $L^{2}(V, \mu)$. Hence

$$
A(x, y)=\sum_{n} a_{n} \overline{\varphi_{n}(x)} \varphi_{n}(y), \quad B(x, y)=\sum_{n} b_{n} \overline{\psi_{n}(x)} \psi_{n}(y)
$$


where $a_{n} \geq 0, b_{n} \geq 0$, and the $\varphi_{n}$ (resp. $\left.\psi_{n}\right)$ are an orthonormal sequence of functions on $V$; they are smooth with compact support since $a_{n} \varphi_{n}(y)=\int_{V} \varphi_{n}(x) A(x, y) d \mu(x)$. We can approximate $A$ by $A_{N}$ (resp. $B$ by $B_{N}$ ) obtained by stopping the expansions (C.19) at $n=N$ and set $C_{N}=A_{N} B_{N}$. Let $f$ be a $\mathcal{C}^{\infty}$ function with compact support on $V$.

$$
\int_{V \times V} \overline{f(x)} C_{N}(x, y) f(y) d \mu(x) d \mu(y)=\sum_{n=1}^{N} \sum_{m=1}^{N} a_{n} b_{m}\left|\int_{V} f(x) \varphi_{n}(x) \psi_{m}(x) d \mu(x)\right|^{2} \geq 0 .
$$

Taking the limit of the lhs as $N \rightarrow \infty$ shows that $C$ is positive-semi-definite.

This lemma can easily be generalized (e.g. to the case of two distributional kernels that happen to be pointwise multipliable, such as Wightman functions) by a regularization-deregularization procedure.

\section{Appendix. Existence of the functions $g_{l}$}

We need to prove, for real $\lambda$ (close to $n$ ), the linear independence of the functions

$$
y \mapsto \varphi(y, n) \quad \text { and } \quad y \mapsto \operatorname{Re} \frac{\partial}{\partial \lambda} \varphi(y, \lambda),\left.\right|_{\lambda=n}, \quad y \in \mathbf{R},
$$

where

$$
\varphi(y, \lambda)=e^{\frac{i \pi}{2}(\lambda-l)}\left(1+y^{2}\right)^{-\frac{l}{2}-\frac{d-2}{4}} \mathbf{P}_{\lambda+\frac{d-2}{2}}^{-l-\frac{d-2}{2}}(-i y),
$$

It has already been seen that $\varphi(y, n)$ is real, hence we must check that $\operatorname{Re} \varphi(y, \lambda)$ and $\operatorname{Re} \frac{\partial}{\partial \lambda} \varphi(y, \lambda)$ are linearly independent at $\lambda=n$. The formula [42, $3.2(22)$, p. 126] gives

$$
\begin{gathered}
\varphi(y, \lambda)=e^{\frac{i \pi}{2}(\lambda-n)}\left[\varphi_{1}(y, \lambda)+\varphi_{2}(y, \lambda)\right], \\
\varphi_{1}(y, \lambda)=\frac{e^{\frac{i \pi}{2}(n-l)} 2^{-l-\kappa} \pi^{1 / 2}}{\Gamma\left(\frac{1+l-\lambda}{2}\right) \Gamma\left(\frac{2+2 \kappa+l+\lambda}{2}\right)} F\left(\frac{l-\lambda}{2}, \frac{1+2 \kappa+l+\lambda}{2} ; \frac{1}{2} ;-y^{2}\right) \\
\varphi_{2}(y, \lambda)=\frac{2 i y e^{\frac{i \pi}{2}(n-l)} 2^{-l-\kappa} \pi^{1 / 2}}{\Gamma\left(\frac{l-\lambda}{2}\right) \Gamma\left(\frac{1+2 \kappa+l+\lambda}{2}\right)} F\left(\frac{1+l-\lambda}{2}, \frac{2+2 \kappa+l+\lambda}{2} ; \frac{3}{2} ;-y^{2}\right) \\
\left.\partial_{\lambda} \varphi(y, \lambda)\right|_{\lambda=n}=\frac{i \pi}{2} \varphi(y, n)+\left.\partial_{\lambda} \varphi_{1}(y, \lambda)\right|_{\lambda=n}+\left.\partial_{\lambda} \varphi_{2}(y, \lambda)\right|_{\lambda=n} .
\end{gathered}
$$

\section{D.1 Case $n-l \geq 0$ even : $n-l=2 N \geq 0, N$ integer}

In this case, $\varphi_{1}(y, \lambda)$ is real, $\varphi_{2}(y, \lambda)$ and $\partial_{\lambda} \varphi_{2}(y, \lambda)$ are pure imaginary, and $\varphi_{2}(y, n)=$,0 . Let $\lambda=n+2 s$ (s real), so that $\partial_{\lambda}=\frac{1}{2} \partial_{s}$.

$$
\begin{gathered}
\varphi_{1}(y, \lambda)=U_{1}(\lambda) V_{1}(y, \lambda), \\
U_{1}(\lambda)=\frac{(-1)^{N} 2^{-l-\kappa} \pi^{1 / 2}}{\Gamma\left(\frac{1}{2}-N-s\right) \Gamma(1+\kappa+l+N+s)} \\
V_{1}(y, \lambda)=F\left(-N-s, \frac{1}{2}+\kappa+l+N+s ; \frac{1}{2} ;-y^{2}\right)
\end{gathered}
$$

Denoting $V_{1,1}(y, \lambda)$ the sum of terms of order $\leq N$ in the expansion of the rhs of (D.9) in powers of $\left(-y^{2}\right)$, and $V_{1,2}(y, \lambda)$ the sum of terms of order $\geq N+1$, we have

$$
\begin{aligned}
& \left.\partial_{\lambda} V_{1,1}(y, \lambda)\right|_{\lambda=n}=\sum_{m=0}^{N} \frac{\Gamma(N+1) \Gamma\left(\frac{1}{2}+\kappa+l+N+m\right) \Gamma\left(\frac{1}{2}\right) y^{2 m}}{2 \Gamma(N+1-m) \Gamma\left(\frac{1}{2}+\kappa+l+N\right) \Gamma\left(\frac{1}{2}+m\right) \Gamma(m+1)} \times \\
& \times\left[\psi(N+1)+\psi\left(\frac{1}{2}+\kappa+l+N+m\right)-\psi(N+1-m)-\psi\left(\frac{1}{2}+\kappa+l+N\right)\right] .
\end{aligned}
$$




$$
\begin{aligned}
& \left.\partial_{\lambda} V_{1,2}(y, \lambda)\right|_{\lambda=n}= \\
& =\sum_{m=N+1}^{\infty} \frac{(-1)^{N+1} N !(m-(N+1)) !}{m !} \frac{\Gamma\left(\frac{1}{2}+\kappa+l+N+m\right) \Gamma\left(\frac{1}{2}\right)\left(-y^{2}\right)^{m}}{2 \Gamma\left(\frac{1}{2}+\kappa+l+N\right) \Gamma\left(\frac{1}{2}+m\right)} .
\end{aligned}
$$

Since

$$
\begin{aligned}
& \varphi(y, n)=\varphi_{1}(y, n)=U_{1}(n) V_{1}(y, n) \\
& \left.\operatorname{Re} \partial_{\lambda} \varphi(y, \lambda)\right|_{\lambda=n}=\left.\partial_{\lambda} U_{1}(\lambda)\right|_{\lambda=n} V_{1}(y, n)+\left.U_{1}(n) \partial_{\lambda} V_{1}(y, \lambda)\right|_{\lambda=n}
\end{aligned}
$$

proving that $\varphi(y, n)$ and $\left.\operatorname{Re} \partial_{\lambda} \varphi(y, \lambda)\right|_{\lambda=n}$ are linearly independent is equivalent to proving that $V_{1}(y, n)$ and $\left.\partial_{\lambda} V_{1}(y, \lambda)\right|_{\lambda=n}$ are linearly independent. This is obvious since $V_{1}(y, n)$ is a polynomial of degree $N$ in $y^{2}$ while the expansion of $\left.\partial_{\lambda} V_{1}(y, \lambda)\right|_{\lambda=n}$ in powers of $y^{2}$ has non-zero terms of higher degree.

\section{D.2 Case $n-l>0$ odd : $n-l-1=2 N \geq 0, N$ integer}

In this case, $\varphi_{2}(y, \lambda)$ is real, $\varphi_{1}(y, \lambda)$ and $\partial_{\lambda} \varphi_{1}(y, \lambda)$ are pure imaginary, and $\varphi_{1}(y, n)=$,0 . Setting $\lambda=n+2 s$ ( $s$ real),

$$
\begin{gathered}
\varphi_{2}(y, \lambda)=y U_{2}(\lambda) V_{2}(y, \lambda), \\
U_{2}(\lambda)=\frac{(-1)^{N+1} 2^{-l-\kappa+1} \pi^{1 / 2}}{\Gamma\left(\frac{1}{2}-N-s\right) \Gamma(1+\kappa+l+N+s)}, \\
V_{2}(y, \lambda)=F\left(-N-s, \frac{3}{2}+\kappa+N+l+s ; \frac{3}{2} ;-y^{2}\right) \\
=V_{2,1}(y, \lambda)+V_{2,2}(y, \lambda) .
\end{gathered}
$$

where $V_{2,1}(y, \lambda)$ is the sum of the terms of order $\leq N$ in the expansion of (D.15) in powers of $\left(-y^{2}\right)$, and $V_{2,2}(y, \lambda)$ is the sum of the terms of order $\geq N+1$.

$$
\begin{gathered}
\left.\partial_{\lambda} V_{2,1}(y, \lambda)\right|_{\lambda=n}=\sum_{m=0}^{N} \frac{\Gamma(N+1) \Gamma\left(\frac{3}{2}+p+l+N+m\right) \Gamma\left(\frac{3}{2}\right) y^{2 m}}{2 \Gamma(N+1-m) \Gamma\left(\frac{3}{2}+p+l+N\right) \Gamma\left(\frac{3}{2}+m\right) \Gamma(m+1)} \times \\
\times\left[\psi(N+1)+\psi\left(\frac{3}{2}+p+l+N+m\right)-\psi(N+1-m)-\psi\left(\frac{3}{2}+p+l+N\right)\right] . \\
\left.\partial_{\lambda} V_{2,2}(y, \lambda)\right|_{\lambda=n}= \\
=\sum_{m=N+1}^{\infty} \frac{(-1)^{N+1} N !(m-(N+1)) !}{m !} \frac{\Gamma\left(\frac{3}{2}+\kappa+l+N+m\right) \Gamma\left(\frac{3}{2}\right)\left(-y^{2}\right)^{m}}{2 \Gamma\left(\frac{3}{2}+\kappa+l+N\right) \Gamma\left(\frac{3}{2}+m\right)}
\end{gathered}
$$

In the same way as in the preceding subsection, it follows that $\varphi(y, n)$ and $\left.\operatorname{Re} \partial_{\lambda} \varphi(y, \lambda)\right|_{\lambda=n}$ are linearly independent. 


\section{E Appendix. Expansions of $W_{\lambda}\left(z_{1}, z_{2}\right)$ and $\widehat{W}_{n}\left(z_{1}, z_{2}\right)$}

\section{E.1 Formulae for $w_{\lambda}$}

In this appendix we regard $w_{\lambda}\left(z_{1} \cdot z_{2}\right)$ as a function of $\lambda, z=(1+\zeta) / 2=-\left(z_{1}-z_{2}\right)^{2} / 4$, and a complex variable $\alpha$ eventually to be set equal to $(d-2) / 2$.

$$
\begin{aligned}
w_{\lambda}\left(z_{1} \cdot z_{2}\right) & =\frac{\Gamma(-\lambda) \Gamma(\lambda+2 \alpha+1)}{(4 \pi)^{\alpha+1} \Gamma(\alpha+1)} F(-\lambda, \lambda+2 \alpha+1 ; \alpha+1 ; 1-z) \\
& =\frac{\Gamma(-\lambda) \Gamma(\lambda+2 \alpha+1) \Gamma(-\alpha)}{(4 \pi)^{\alpha+1} \Gamma(\lambda+\alpha+1) \Gamma(-\lambda-\alpha)} F(-\lambda, \lambda+2 \alpha+1 ; \alpha+1 ; z)+ \\
& +\frac{\Gamma(\alpha) z^{-\alpha}}{(4 \pi)^{\alpha+1}} F(\lambda+\alpha+1,-\lambda-\alpha ; 1-\alpha ; z) .
\end{aligned}
$$

We are interested in the behavior of $w_{\lambda}$ in the neighborhood of $z=0$, which is made explicit by (E.2) unless $\alpha$ is an integer. This happens if $d$ is a positive even integer and one attempts to set $\alpha=(d-2) / 2$. This is the case on which we will focus our attention. In this appendix $n$ will denote a fixed non-negative integer.

In addition to the well-known identity

$$
\Gamma(u) \Gamma(1-u)=\pi / \sin (\pi u)
$$

we will need the following formulae, where $\psi(u)=\Gamma^{\prime}(u) / \Gamma(u)$ and $p \geq 0$ is an integer:

$$
\begin{gathered}
\frac{\psi(u)}{\Gamma(u)}=\frac{\Gamma^{\prime}(u)}{\Gamma(u)^{2}}=-\frac{\partial}{\partial u} \Gamma(u)^{-1}=-\frac{\partial}{\partial u} \pi^{-1} \sin (\pi u) \Gamma(1-u) \\
=-\cos (\pi u) \Gamma(1-u)+\pi^{-1} \sin (\pi u) \Gamma^{\prime}(1-u), \\
\left.\frac{\psi(u)}{\Gamma(u)}\right|_{u=-p}=-p ! \cos (p \pi)=(-1)^{p+1} p ! ; \\
\frac{\partial}{\partial u}\left[\frac{\psi(u)}{\Gamma(u)}\right]=\pi \sin (\pi u) \Gamma(1-u)+2 \cos (\pi u) \Gamma^{\prime}(1-u)-\pi^{-1} \sin (\pi u) \Gamma^{\prime \prime}(1-u), \\
\left.\frac{\partial}{\partial u}\left[\frac{\psi(u)}{\Gamma(u)}\right]\right|_{u=-p}=2 \cos (p \pi) \Gamma^{\prime}(1+p)=2(-1)^{p} p ! \psi(1+p),
\end{gathered}
$$

and, for any $x \in \mathbf{C}$,

$$
\frac{\Gamma(-x+p)}{\Gamma(-x)}=\frac{(-1)^{p} \Gamma(x+1)}{\Gamma(x-p+1)}
$$

\section{E.2 Pole removal procedure at $\lambda=n$}

In sect. $5 \widehat{w}_{n}\left(z_{1} \cdot z_{2}\right)$ was defined by applying a "pole removal procedure" (PRP) to $w_{\lambda}\left(z_{1} \cdot z_{2}\right)$. This procedure can be applied to any meromorphic function $f$ that has (at worst) a simple pole at $n$ :

$$
\widehat{f}(n)=\left.\frac{(-1)^{n+1}}{n !} \frac{\partial}{\partial \lambda}\left(\frac{f(\lambda)}{\Gamma(-\lambda)}\right)\right|_{\lambda=n} .
$$

Taking

$$
f(\lambda)=\frac{a}{\lambda-n}+h(\lambda)
$$

where $h$ is holomorphic at $n$, one gets

$$
\widehat{f}(n)=a \psi(1+n)+h(n) .
$$

In particular if $a=0$, i.e. if $f$ is actually holomorphic at $n$, then $\widehat{f}(n)=f(n)$, i.e. the procedure does nothing. Operating with another function than $\Gamma(-\lambda)$ would simply change the coefficient of $a$. 


\section{E.3 The case of even dimension}

We fix an integer $N \geq 0$. If $\alpha$ tends to $N$, the first form (E.1) is regular, but each of the two terms in (E.2) has a pole in $\alpha$. There must therefore be a cancellation of the poles in the two terms of (E.2). This may also be understood by representing the value of (E.1) by a Cauchy integral (in the variable $\alpha$ ) along a small circle centered at $N$. On this contour both terms in (E.2) are regular and the two hypergeometric functions may be expanded into their power series at 0 . We will use a more explicit way of exhibiting the cancellation, but the preceding remark shows that the forthcoming expansions are uniformly convergent in $\mathbf{U} \stackrel{\text { def }}{=}\{z:|z|<1\}$.

Expanding the two hypergeometric functions in powers of $z$ shows that, near $\alpha=N$,

$$
w_{\lambda}\left(z_{1} \cdot z_{2}\right)=\sum_{m=0}^{N-1} c_{m}(\lambda, \alpha) z^{m-\alpha}+g(\lambda, \alpha) \frac{f_{1}(\lambda, \alpha, z)+f_{2}(\lambda, \alpha, z)}{\sin (\pi \alpha)},
$$

where (using (E.6)

$$
\begin{gathered}
c_{m}(\lambda, \alpha)=\frac{(-1)^{m} \Gamma(\alpha-m) \Gamma(\lambda+\alpha+1+m) \Gamma(-\lambda-\alpha+m)}{(4 \pi)^{\alpha+1} \Gamma(\lambda+\alpha+1) \Gamma(-\lambda-\alpha) m !}, \\
g(\lambda, \alpha)=\frac{\pi}{(4 \pi)^{\alpha+1} \Gamma(\lambda+\alpha+1) \Gamma(-\lambda-\alpha)}, \\
f_{1}(\lambda, \alpha, z)=-\sum_{m=0}^{\infty} \frac{z^{m} \Gamma(-\lambda+m) \Gamma(\lambda+2 \alpha+1+m)}{\Gamma(\alpha+1+m) \Gamma(m+1)}, \\
f_{2}(\lambda, \alpha, z)=\sum_{m=0}^{\infty} \frac{z^{m+N-\alpha} \Gamma(\lambda+\alpha+1+m+N) \Gamma(-\lambda-\alpha+m+N)}{\Gamma(1-\alpha+m+N) \Gamma(m+N+1)} .
\end{gathered}
$$

All of these functions are regular in $\alpha$ near $N$, and $f_{1}(\lambda, N, z)+f_{2}(\lambda, N, z)=0$. Hence

$$
\left.w_{\lambda}\left(z_{1} \cdot z_{2}\right)\right|_{\alpha=N}=\sum_{m=0}^{N-1} c_{m}(\lambda, N) z^{m-N}+g(\lambda, N)\left[\frac{\partial_{\alpha} f_{1}(\lambda, N, z)+\partial_{\alpha} f_{2}(\lambda, N, z)}{\pi \cos (\pi N)}\right] .
$$

With the help of (E.6) this gives

$$
\begin{aligned}
& w_{\lambda}\left(z_{1} \cdot z_{2}\right)=\sum_{m=0}^{N-1} \frac{z^{m-N} \Gamma(N-m) \Gamma(\lambda+N+1+m)}{(4 \pi)^{N+1} \Gamma(\lambda+N-m+1) m !}+ \\
& +\sum_{m=0}^{\infty} \frac{(-1)^{m} z^{m} \Gamma(\lambda+2 N+1+m)}{(4 \pi)^{N+1} \Gamma(1+m) \Gamma(N+1+m) \Gamma(\lambda-m+1)} \times \\
& {[-\log z+\psi(1+m)-\psi(\lambda+2 N+1+m)+\psi(N+1+m)-\psi(-\lambda+m)] .}
\end{aligned}
$$

\section{E.3.1 Applying the pole removal procedure at $\lambda=n$}

Only the terms in (E.14) containing $\psi(-\lambda+m)$, with $m \leq n$ fail to be analytic in $\lambda$ at the value $n$. All terms in the infinite series with $m>n$ vanish at $\lambda=n$. Using (E.4), (E.5), and (E.6), it is straightforward to apply the procedure to the relevant terms, and we obtain:

$$
\begin{aligned}
& \widehat{w}_{n}\left(z_{1} \cdot z_{2}\right)=\sum_{m=0}^{N-1} \frac{z^{m-N} \Gamma(N-m) \Gamma(n+N+1+m)}{(4 \pi)^{N+1} \Gamma(n+N+1-m) \Gamma(m+1)}+ \\
& +\sum_{m=0}^{n} \frac{(-1)^{m} z^{m} \Gamma(n+2 N+1+m)}{(4 \pi)^{N+1} \Gamma(1+m) \Gamma(N+1+m) \Gamma(n-m+1)} \times \\
& \times[-\log z+\psi(1+m)+\psi(N+1+m)-2 \psi(n+2 N+1+m)-\psi(1+n)] .
\end{aligned}
$$

Of course this identity extends to the whole cut-plane $\mathbf{C} \backslash \mathbf{R}_{-}$. By using (E.6), the coefficient of $-\log z$ can be rewritten as

$$
\frac{\Gamma(n+2 N+1)}{(4 \pi)^{N+1} \Gamma(N+1) \Gamma(n+1)} F(-n, n+2 N+1 ; N+1 ; z) .
$$


Thus, for even dimension $d \geq 2$,

$$
\widehat{w}_{n}\left(z_{1} \cdot z_{2}\right)=z^{1-\frac{d}{2}} A(z, n, d)-\log (z) B(z, n, d)+C(z, n, d),
$$

where $A, B, C$ are polynomials in $z$ :

$$
\begin{aligned}
A(z, n, d) & =\sum_{m=0}^{\frac{d}{2}-2} \frac{z^{m} \Gamma\left(\frac{d}{2}-1-m\right) \Gamma\left(n+\frac{d}{2}+m\right)}{(4 \pi)^{\frac{d}{2}} \Gamma\left(n+\frac{d}{2}-m\right) m !} \\
B(z, n, d) & =\frac{\Gamma\left(\frac{d-1}{2}\right)}{4 \pi^{\frac{d+1}{2}}} C_{n^{\frac{d-1}{2}}}(1-2 z) \\
C(z, n, d) & =\sum_{m=0}^{n} \frac{(-1)^{m} z^{m} \Gamma(n+d-1+m)}{(4 \pi)^{\frac{d}{2}} \Gamma(1+m) \Gamma\left(\frac{d}{2}+m\right) \Gamma(n-m+1)} \times \\
& \times\left[\psi(1+m)+\psi\left(\frac{d}{2}+m\right)-2 \psi(n+d-1+m)-\psi(1+n)\right]
\end{aligned}
$$

\section{References}

[1] J. Bros, H. Epstein and U. Moschella, "Scalar tachyons in the de Sitter universe," Lett. Math. Phys. 93, 203 (2010) arXiv:1003.1396 [hep-th]].

[2] G. Börner G. and H.P Dürr, Classical and quantum fields in de Sitter space, Il Nuovo Cimento A LXIV: 669-714 (1969).

[3] B. Allen and A. Folacci, "The Massless Minimally Coupled Scalar Field in De Sitter Space," Phys. Rev. D 35, 3771 (1987).

[4] A. Vilenkin and L. H. Ford, "Gravitational effects upon cosmological phase transition", Phys. Rev. D26 1231, (1982).

[5] A. A. Starobinsky, "Dynamics of phase transition in the new inflationary universe scenario and generation of perturbations", Phys. Lett. 117B (1982) 175.

[6] P. Mazur and E. Mottola, "Spontaneous Breaking of De Sitter Symmetry by Radiative Effects," Nucl. Phys. B 278, 694 (1986).

[7] I. Antoniadis and E. Mottola, "Graviton Fluctuations in De Sitter Space," J. Math. Phys. 32, 1037 (1991).

[8] I. Antoniadis and E. Mottola, "4-D quantum gravity in the conformal sector," Phys. Rev. D 45, 2013 (1992).

[9] A. Folacci, "Toy model for the zero mode problem in the conformal sector of de Sitter quantum gravity," Phys. Rev. D 53, 3108 (1996).

[10] A. Folacci: Zero modes, euclideanization and quantization. Phys. Rev. D46, 2553 (1992).

[11] A. Higuchi, D. Marolf and I. A. Morrison, "de Sitter invariance of the dS graviton vacuum," Class. Quant. Grav. 28, 245012 (2011) arXiv:1107.2712 [hep-th]].

[12] M. Faizal and A. Higuchi, "Physical equivalence between the covariant and physical graviton two-point functions in de Sitter spacetime," Phys. Rev. D 85 (2012) 124021 arXiv:1107.0395 [gr-qc]].

[13] P. J. Mora, N. C. Tsamis and R. P. Woodard, "Graviton Propagator in a General Invariant Gauge on de Sitter," J. Math. Phys. 53, 122502 (2012)

[14] I. A. Morrison, "On cosmic hair and "de Sitter breaking" in linearized quantum gravity," arXiv:1302.1860 [gr-qc].

[15] S. P. Miao, P. J. Mora, N. C. Tsamis and R. P. Woodard, "The Perils of Analytic Continuation," arXiv:1306.5410 [gr-qc].

[16] S. P. Miao, N. C. Tsamis and R. P. Woodard, "De Sitter Breaking through Infrared Divergences," J. Math. Phys. 51, 072503 (2010) arXiv:1002.4037 [gr-qc]]. 
[17] R. F. Streater and A. S. Wightman PCT, Spin and statistics and all that. Princeton University Press, Princeton, 1964.

[18] M. Reed and B. Simon, Methods of Modern Mathematical Physics, Vol. 2. Academic Press (1975)

[19] F. Strocchi and A. S. Wightman, "Proof of the Charge Superselection Rule in Local Relativistic Quantum Field Theory.," J. Math. Phys. 15, 2198 (1974) [Erratum-ibid. 17, 1930 (1976)].

[20] F. Strocchi, "Selected topics on the general properties of quantum field theory," World Sci. Lect. Notes Phys. 51, 1 (1993).

[21] B. Schroer, "The quantization of $m^{2}<0$ field equations," Phys. Rev. D 3, 1764 (1971).

[22] P. R. Anderson, W. Eaker, S. Habib, C. Molina-Paris and E. Mottola, Phys. Rev. D 62, 124019 (2000) gr-qc/0005102.

[23] A. Youssef, "Do scale-invariant fluctuations imply the breaking of de Sitter invariance?," Phys. Lett. B 718, 1095 (2013) arXiv:1203.3171 [gr-qc]].

[24] G. Feinberg, "Possibility of Faster-Than-Light Particles". Physical Review 159, 5 (1967).

[25] J.Bros, J. P. Gazeau and U. Moschella, "Quantum field theory in the de Sitter universe," Phys. Rev. Lett. 73 (1994) 1746.

[26] S. Deser and A. Waldron, "Partial masslessness of higher spins in (A)dS," Nucl. Phys. B 607 (2001) 577 hep-th/0103198.

[27] S. Deser and A. Waldron, "Arbitrary spin representations in de Sitter from dS / CFT with applications to dS supergravity," Nucl. Phys. B 662, 379 (2003) hep-th/0301068.

[28] J. Bros and U. Moschella, "Two point functions and quantum fields in de Sitter universe," Rev. Math. Phys. 8, 327 (1996) gr-qc/9511019.

[29] J. Bros, H. Epstein and U. Moschella, "Analyticity properties and thermal effects for general quantum field theory on de Sitter space-time," Commun. Math. Phys. 196, 535 (1998) gr-qc/9801099.

[30] J. Faraut, "Noyaux sphériques sur un hyperboloïde à une nappe" in Lect. Notes in Math. 497, Springer-Verlag, Berlin (1975).

[31] Molčanov V.F.: "Harmonic analysis on a hyperboloid of one sheet", Soviet. Math. Dokl. 7, 1553-1556 (1966)

[32] W. Thirring: Quantum field theory in de Sitter space. Acta Physica Austriaca, suppl. IV, 1967, 269.

[33] O. Nachtmann: Dynamische Stabilität im de-Sitter-raum. Österr. Akad. Wiss., Math.-Naturw. Kl., Abt. II 176:363-379, 1968.

[34] N. A. Chernikov and E. A. Tagirov. Quantum theory of scalar fields in de Sitter space-time. Annales Poincare. Phys. Theor., A9:109, (1968).

[35] C. Schomblond and P. Spindel, "Conditions d'unicité pour le propagateur $\Delta^{1}(x, y)$ du champ scalaire dans l'univers de de Sitter", Annales Poincare Phys. Theor. 25, 67 (1976).

[36] G. W. Gibbons and S. W. Hawking. Cosmological Event Horizons, Thermodynamics, and Particle Creation. Phys. Rev., D15:2738-2751, 1977.

[37] T.S. Bunch, P.C.W. Davies: Proc. R.Soc. Lond. A 360, 117 (1978).

[38] E. Mottola, Particle creation in de Sitter space, Phys. Rev. D. 31, 754, (1985).

[39] B. Allen, "Vacuum States in de Sitter Space," Phys. Rev. D 32, 3136 (1985).

[40] A. Erdélyi. The Bateman manuscript project. Higher Transcendental Functions, volume II. McGraw-Hill, New York, 1953.

[41] N.Ja. Vilenkin. Special Functions and the Theory of Group Representations. Nauka, Moscow, 1968.

[42] A. Erdélyi. The Bateman manuscript project. Higher Transcendental Functions, volume I. McGraw-Hill, New York, 1953. 
[43] J. Dimock, Algebras of local observables on a manifold Comm. Math. Phys. 77, 211-297 (1980).

[44] R.M. Wald. Quantum field theory in curved space-time and black hole thermodynamics. Chicago University Press. (1995).

[45] U. Moschella, R. Schaeffer Quantum fields on curved spacetimes and a new look at the Unruh effect, AIP Conf.Proc. 1132, 303-332, (2009)

[46] S. P. Miao, N. C. Tsamis and R. P. Woodard, "The Graviton Propagator in de Donder Gauge on de Sitter Background," J. Math. Phys. 52, 122301 (2011) arXiv:1106.0925 [gr-qc]].

[47] I. M. Gelfand, M. I. Graev and N. Ya. Vilenkin, Generalized functions. Vol. 5: Integral geometry and representation theory, Boston, MA: Academic Press (1966)

[48] J. Bros and U. Moschella, "Fourier analysis and holomorphic decomposition on the one sheeted hyperboloid," math-ph/0311052. In: F. Norguet, S. Ofman et J.-J. Szczeciniarz. Géométrie complexe II. p. 100-145, Paris: Hermann (2003).

[49] A. M. Polyakov, "De Sitter space and eternity," Nucl. Phys. B 797, 199 (2008) arXiv:0709.2899 [hep-th]].

[50] A. M. Polyakov, "Infrared instability of the de Sitter space," arXiv:1209.4135 [hep-th].

[51] P. R. Anderson and E. Mottola, "Quantum Vacuum Instability of 'Eternal' de Sitter Space," arXiv:1310.1963 [gr-qc].

[52] P. R. Anderson and E. Mottola, "On the Instability of Global de Sitter Space to Particle Creation," arXiv:1310.0030 [gr-qc].

[53] E. T. Akhmedov, "Lecture notes on interacting quantum fields in de Sitter space," Int. J. Mod. Phys. D 23, 1430001 (2014) arXiv:1309.2557 [hep-th]].

[54] Morchio, G.; Strocchi, F. Infrared singularities, vacuum structure and pure phases in local quantum field theory. Annales de l'institut Henri Poincaré (A) Physique théorique, 33 no. 3 (1980), p. 251-282

[55] G. Morchio, D. Pierotti, and F. Strocchi: Infrared And Vacuum Structure In Two-dimensional Local Quantum Field Theory Models. The Massless Scalar Field, J.Math.Phys. 311467 (1990)

[56] M. Bertola, F. Corbetta and U. Moschella. Massless scalar field in two-dimensional de Sitter universe. Prog. Math. 251, 27 (2007) arXiv:math-ph/0609080.

[57] S.W. Hawking, G.F.R. Ellis. The Large scale structure of space-time Cambridge University Press, Cambridge, 1973

[58] M. Mehta. Matrix Theory. Les Editions de Physique, Les Ulis, 1989.

[59] G. Szegö. Orthogonal Polynomials. Amer. Math. Soc, Providence, RI, 1975. 